Aus der Abteilung Orthopädie

(Prof. Dr. med. W. Schultz)

im Zentrum Chirurgie

der Medizinischen Fakultät der Universität Göttingen

\title{
Lebensqualität und Gelenkfunktion nach Knorpel-Knochen-Transplantation
}

\author{
Langzeitergebnisse der autologen \\ Knorpel-Knochen-Transplantation am Kniegelenk
}

\author{
INAUGURAL - DISSERTATION \\ zur Erlangung des Doktorgrades \\ der Medizinischen Fakultät \\ der Georg-August-Universität zu Göttingen
}

vorgelegt von
Sven Freche
aus
Lauchhammer

Göttingen 2010 
De k a n:

Prof. Dr. med. C. Frömmel

I. Berichterstatter: $\quad$ Prof. Dr. med. W. Schultz

II. Berichterstatter/in: Priv. - Doz. Dr. med. Frosch

III. Berichterstatter/in:

Tag der mündlichen Prüfung: $\quad$ 23.06.2010 
1. EINLEITUNG

1.1 ANATOMIE UND FUNKTION DES KNIEGELENKES 4

1.1.1 Makroskopische Anatomie 4

1.1.2 Gelenkknorpel 6

$\begin{array}{ll}\text { 1.1.3 Biomechanik } & 10\end{array}$

1.2 PATHOLOGIE DES KNORPELS 11

1.2.1 Epidemiologie des Knorpelschadens 11

1.2.2 Ätiologie des Knorpelschadens 12

1.2.3 Pathomechanik und Pathogenese 12

$\begin{array}{ll}\text { 1.2.4 Klinik } & 14\end{array}$

$\begin{array}{ll}1.3 \text { DIAGNOSTIK } & 15\end{array}$

1.3.1 Anamnese und Klinische Untersuchung 15

1.3.2 Konventionelles Röntgen $\quad 15$

1.3.3 Magnetresonanztomographie 16

$\begin{array}{ll}\text { 1.3.4 Arthroskopie } & 18\end{array}$

$\begin{array}{ll}1.4 \text { THERAPIE } & 21\end{array}$

1.4.1 konservative Therapie $\quad 21$

1.4.2 operative Therapie 22

1.4.2.1 Regenerative Strategien 22

1.4.2.2 Gewebeersatz 23

1.4.2.3 Tissue Engineering 24

1.4.3 Therapiealgorithmus $\quad 25$

1.4.4 Rehabilitation nach Knorpel-Knochen-Transplantation 26

1.4.5 Prognose nach Knorpel-Knochen-Transplantation 26

2. HYPOTHESE $\quad 27$

3. MATERIAL UND METHODEN 28

3.1 EIN- UND AUSSCHLUSSKRITERIEN 28

3.2 AUSWERTUNGSSKALEN 28

3.2.1 SF-36 29

3.2.2 IKDC 31

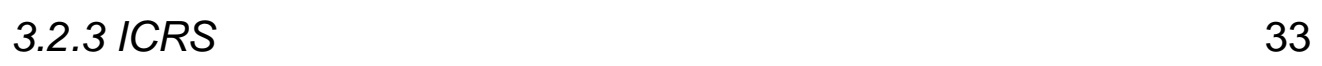

3.3 PATIENTENKOLLEKTIV 34

$\begin{array}{ll}3.4 \text { STATISTIK } & 39\end{array}$ 
4. ERGEBNISSE $\quad 40$

4.1 ERGEBNISSE DES SF-36-FRAGEBOGENS 40

4.2 ERGBENISSE DES IKDC-FRAGEBOGENS 47

4.3 ERGEBNISSE DES ICRS-BEURTEILUNGSBOGENS 50

5. DISKUSSION

$\begin{array}{ll}\text { 6. ZUSAMMENFASSUNG } & 60\end{array}$

7. ANHANG : ICRS-BEURTEILUNGSPAKET 65

8. LITERATURVERZEICHNIS $\quad 80$ 


\section{ABKÜRZUNGSVERZEICHNIS}

$\mathrm{ACl}$

AGES

ANOVA

a. p.

$\mathrm{BMI}$

CPM

DMOAD

ECM

EMRO

FSE

GAG

HE

ICRS

IKDC

IL

KÖFU

KÖRO

KÖSC

$\mathrm{kp}$

LFC

Lig.

Ligg.

$\mathrm{M}$.

$\mathrm{MACl}$

Mega-OATS $^{\circledR}$

MFC

ML

MRT

NSAR

OATS $^{\circledR}$

PD

PSYC
Autologous Chondrocyte Implantation

Allgemeine Gesundheit

Analysis Of Variance

anterior - posterior

Body-Mass-Index

Continuous Passive Motion

Disease Modifying Osteoarthritis Drug

Extrazelluläre Matrix

Emotionale Rollenfunktion

Fast-Spin-Echo

Glykosaminoglykane

Hämatoxylin-Eosin

International Cartilage Repair Society

International Knee Documentation Committee

Interleukin

Körperliche Funktionsfähigkeit

Körperliche Rollenfunktion

Körperliche Schmerzen

Kilopond

Lateraler Femurkondylus

Ligament

Ligamenta

Musculus

Matrix-Associated Autologous Chondrocyte Implantation

Mega-Osteochondral Autograft Transfer System

Medialer Femurkondylus

Meniskusläsion

Magnetresonanztomographie

Nicht-Steroidale Antirheumatika

Osteochondral Autograft Transfer System

Patellare Dislokation

Psychisches Wohlbefinden 
Abkürzungsverzeichnis

SADAO

SF-36

SK

SOFU

$\mathrm{T}$

VITA

VKB

ZMK
Slow Acting Drugs in Osteoarthritis

Short-Form health survey

Subchondraler Knochen

Soziale Rollenfunktion

Tesla

Vitalität

Vorderes Kreuzband

Zone des mineralisierten Knorpels 


\section{ABBILDUNGSVERZEICHNIS}

Abb. 1 : Anatomie des Kniegelenkes I 4

Abb. 2 : Anatomie des Kniegelenkes II 5

Abb. 3 : Molekularer Aufbau der Knorpelmatrix $\quad 7$

Abb. 4.a: Zonen des Gelenkknorpels schematisch 8

Abb. 4.b: Zonen des gesunden Gelenkknorpels 8

Abb. 5 : MRT-Studienprotokoll (FSE) 16

Abb. 6 : MRT des rechten Kniegelenkes mit sog. „Bone-Bruise“ 17

Abb. 7 : Arthroskopischer Normalbefund $\quad 19$

Abb. 8 : Knorpelschaden Grad II nach Outerbridge 19

Abb. 9 : Knorpelschaden Grad IV nach Outerbridge 19

Abb. 10 : Klassifikation des Knorpelschadens nach ICRS 20

Abb. 11 : Arthroskopischer Befund nach Pridie-Bohrung 22

Abb. 12 : OP-Situs am medialen Femurkondylus 23

Abb. 13 : Transformationsalgorithmus der Skalenwerte des SF- $36 \quad 30$

Abb. 14 : Transformationsalgorithmus der Skalenwerte des IKDC-Rohwertes 31

Abb. 15 : Aktivität der Patienten zum Zeitpunkt der Verletzung 34

Abb. 16 : Zeitraum zwischen Schmerzereignis bzw. Trauma und OP in Tagen 34

Abb. 17 : Patientenanzahl nach Altersgruppen zum Operationszeitpunkt 35

Abb. 18 : Patientenanzahl nach Body-Mass-Index zum Operationszeitpunkt 35

Abb. 19 : Lokalisationen der osteochondralen Läsion 36

Abb. 20 : Größe nach Debridement 36

Abb. 21 : Zeitraum der Nachuntersuchung $\quad 37$

Abb. 22 : prä- und postoperative Werte der Subskalen des SF-36 41

Abb. 23 : prä- und postoperative Werte der Summenskalen des SF-36 41

Abb. 24 : postoperative Subskalenwerte im Vergleich mit der deutschen Normbevölkerung 43

Abb. 25 : postoperative Summenskalenwerte im Vergleich mit der deutschen Normbevölkerung 43

Abb. 26 : Korrelation der Werte KÖFU und des BMI 45

Abb. 27 : Korrelation der Werte EMRO mit den Werten des BMI 45

Abb. 28 : Korrelation der Werte PSYC mit der Defektgröße 46

Abb. 29 : Gesundheitsveränderung 46 
Abb. 30 : Vergleich der prä- und postoperativen Punktwerte des IKDC

Abb. 31 : Veränderung des IKDC-Wertes je nach Fall

Abb. 32 : prä- und postoperative Gruppengrade des IKDC

Abb. 33 : Korrelation zwischen IKDC-Veränderung und BMI

Abb. 34 : Vergleich prä- und postoperativer ICRS-Wert

Abb. 35 : Vergleich der präoperativen klinischen Punktwerte des IKDC 56

Abb. 36 : Vergleich der postoperativen klinischen Punktwerte des IKDC 56

Abb. 37 : Vergleich der postoperativen Punktwerte des ICRS

Abb. 38 : ICRS-Beurteilungspaket, S. 1

Abb. 39 : ICRS-Beurteilungspaket, S. 2

Abb. 40 : ICRS-Beurteilungspaket, S. 3

Abb. 41 : ICRS-Beurteilungspaket, S. 4 68

Abb. 42 : ICRS-Beurteilungspaket, S. 5 69

Abb. 43 : ICRS-Beurteilungspaket, S. 6 70

Abb. 44 : ICRS-Beurteilungspaket, S. 7 71

Abb. 45 : ICRS-Beurteilungspaket, S. 8 72

Abb. 46 : ICRS-Beurteilungspaket, S. 9 73

Abb. 47 : ICRS-Beurteilungspaket, S. 10 74

Abb. 48 : ICRS-Beurteilungspaket, S. 11 75

Abb. 49 : ICRS-Beurteilungspaket, S. 12 76

Abb. 50 : ICRS-Beurteilungspaket, S. 13 77

Abb. 51 : ICRS-Beurteilungspaket, S. 14 78

Abb. 52 : ICRS-Beurteilungspaket, S. 15

\section{TABELLENVERZEICHNIS}

Tab. 1 : Zonen des Gelenkknorpels $\quad 7$

Tab. 2 : Vergleich der Ergebnisse zur Prävalenz von Knorpelschäden 11

Tab. 3 : Pathomorphologisches Substrat der Knorpelverletzung 13

Tab. 4 : MRT-Klassifikation des Knorpelschadens 17

Tab. 5 : Klassifikation des Knorpelschadens nach Outerbridge 19

Tab. 6 : Subskalen des SF-36 29

Tab. 7 : Kodierung der Fragen zur Bearbeitung mit SPSS 30 
Tab. 8 : Fragenkategorien zur subjektiven Funktionsbeurteilung

Tab. 9 : Eingriffe und zusätzliche operative Maßnahmen 36

$\begin{array}{ll}\text { Tab. } 10 \text { : Patientendaten } & 37\end{array}$

Tab. 11 : Gesamtdatenlage des Patientenkollektivs 38

Tab. 12 : Prä- und postoperative Daten der Sub- und Summenskalen des SF-36 40

Tab. 13 : Postoperative Sub- und Summenskalen im Vergleich mit der deutschen Normbevölkerung 42

Tab. 14: Veränderung der prä- und postoperativen Punktwerte der Subskalen $\quad 44$

Tab. 15: Prä- und postoperative Punktwerte des IKDC 47

Tab. 16: Veränderung der Mittelwerte des IKDC zwischen zwei BMI-Gruppen 49

Tab. 17: Vergleich der Mittelwerte der Altersgruppe der 31- 40jährigen mit dem Patientenkollektiv 


\section{EINLEITUNG}

Die Weltgesundheitsorganisation hat das letzte Jahrzehnt den Erkrankungen des Stütz- und Bewegungsapparates („Bone and Joint Decade“) gewidmet (Weinstein 2000). Dies unterstreicht den Stellenwert von Erkrankungen des Bewegungsapparates und deren Behandlung in der medizinischen Praxis.

In 10 bis $12 \%$ der Gesamtbevölkerung sind Knorpelschäden existent (Sellards et al. 2002). Eine amerikanische Studie zeigt, dass jährlich ca. eine Million Amerikaner von Knorpelschäden betroffen sind (Cole et al. 1999). Zur Behandlung von Knorpeldefekten werden demnach beispielsweise in den USA jährlich mehr als 200.000 operative Eingriffe durchgeführt. Vergleicht man die Studiendaten hinsichtlich der Inzidenz von Knorpelschäden am Kniegelenk, so bestehen bei Patienten mit Kniegelenkbeschwerden in ca. 60 \% Knorpelschäden (Curl et al. 1997, Hjelle et al. 2002, Årøen et al. 2004, Figueroa et al. 2007, Widuchowski et al. 2007).

Hempfling und Weise veröffentlichten 2007 eine Arbeit zur Begutachtung des Knorpelschadens. Diese zeigt, dass eine Unterscheidung zwischen traumatischem und nicht-traumatischem Knorpelschaden notwendig ist. In der Begutachtung des Knorpelschadens wird zwischen unfallbedingtem und abnutzungsbedingtem Schaden differenziert. Ein unfallbedingter (traumatischer) Schaden entspricht einer frischen Knorpelverletzung. Ein auslösendes Ereignis geht voraus. Pathomorphologisch können hier beispielsweise Knorpelprellung, Knorpelkontusion, Knorpelfissur oder Knorpelfraktur vorliegen. Der abnutzungsbedingte Knorpelschaden (nichttraumatisch) ist daher zwingend vom unfallbedingten Schaden abzugrenzen. Pathomorphologisch spielen hier neben dem Knorpelschaden Charakteristika wie subchondrale Knochenbeteiligung, Osteophytenformation und Synovialitis eine entscheidende Rolle (Freemont 2006). Der nicht-traumatische Knorpelschaden verläuft im Vergleich zum traumatischen Knorpelschaden stadienhaft. Die Knorpelverletzung ist jedoch als ätiologischer Faktor in der Entstehung des abnutzungsbedingten Knorpelschadens zu werten (Hempfling und Weise 2007). Entsprechend der Ätiologie des Knorpelschadens unterscheidet sich das Vorgehen in Diagnostik und Therapie. 
Die Regenerationsfähigkeit des Knorpelgewebes aus dem Perichondrium endet mit dem Eintreten des Erwachsenenalters (Geneser 1990). Hunter erkannte schon im 18. Jahrhundert, dass sich zerstörter Knorpel nicht mehr aufbauen kann (Hunter 1995). Daher zählt die Behandlung der Knorpelschäden heute grundlegend zum Spektrum der Orthopädie und Unfallchirurgie. Die Klassifikation von Knorpelverletzungen/-schäden erfolgt magnetresonanztomographisch (Vallotton et al. 1995) oder arthroskopisch (z.B. nach Outerbridge, Bauer und Jackson oder ICRS). Wegbereiter wie Johnson, Pridie, Steadman, Hangody, Brittberg und Petersson entwickelten verschiedene Strategien zur stadiengerechten Therapie von Knorpelschäden, siehe im Folgenden.

Als operative Verfahren werden beispielsweise knochenmarkstimulierende Techniken wie die Abrasions-Arthroplastik (Johnson 1986), die Pridie-Bohrung (Pridie 1959) oder die Mikrofrakturierung (Steadman et al. 1997) angewandt. Im speziellen Fall kann auch eine Dissekatrefixierung (mittels Schrauben oder Fibrinkleber) sinnvoll sein (Bruns 1996).

Andererseits besteht die Möglichkeit der Implantation von künstlichen KnorpelImplantaten bzw. der Einsatz der autologen Knorpelzelltransplantation (Brittberg et al. 1994) oder der autologen Knorpel-Knochen-Transplantation (Kish et al. 1999).

Die Knorpel-Knochen-Transplantation stellt eine spezielle Methode dar, bei der osteochondrale Stanzzylinder aus unbelasteten Gelenkbezirken entnommen und in den defekten Teil einbracht werden (Hangody et al. 2004).

Die Behandlung von Knorpeldefekten hat das Ziel, eine schmerzfreie Gelenkfunktion wiederherzustellen. Entscheidend sind die Regeneration des hyalinen Knorpels im Defektareal und die Integration des Knorpels in das umliegende Knorpelgewebe sowie den subchondralen Knochen (Engebretsen und Bahr 2004). Primäres Ziel der operativen Therapie ist die bestmögliche Rekonstruktion der Gelenkfläche in Ihrer ehemaligen Kongruenz, Beschaffenheit und Funktion und somit die Vermeidung von Degeneration und frühzeitiger Arthrose (Fritz et al. 2003).

Der therapeutische Langzeit-Effekt der Knorpel-Knochen-Transplantation (Nachuntersuchungszeitraum bis zu 9,3 Jahren) wurde bisher in nur wenigen Studien beurteilt (Laprell und Petersen 2001, Hangody und Füles 2002, Szerb et al. 2005, Marcacci et al. 2007). 
Als Instrumente zur Objektivierung dienten standardisierte Fragebögen (BrittbergScore, Tegner-Aktivitätsscore, Lysholm-Score, IKDC-Score, ICRS-Score).

Diese Studien zeigen, dass die Knorpel-Knochen-Transplantation kurz- und mittelfristig eine Behandlungsalternative mit gutem Therapieerfolg beim Vorliegen von III.- und IV.gradigen Knorpelschäden darstellt. Die Erarbeitung von Langzeitergebnissen ist jedoch notwendig, um spezielle Aussagen über den dauerhaften Therapieeffekt der Knorpel-Knochen-Transplantation (insbesondere in Hinblick auf die Lebensqualität und Gelenkfunktion) zu ermöglichen.

Fragestellung der folgenden Untersuchungen ist die Beurteilung des klinischen Langzeit-Therapieeffektes der autologen Knorpel-Knochen-Transplantation am Kniegelenk bei einem Nachuntersuchungszeitraum von bis zu 9,3 Jahren. Als Messinstrument dient der ICRS-Evaluierungs-Fragebogen. Es wurden prä- und postoperative Daten über die Selbstzufriedenheit der Patienten, die Veränderung der klinischen Symptomatik, des klinischen Untersuchungsbefundes sowie über die Knorpelstruktur erhoben.

Zusätzlich wird untersucht, ob die Defektgröße, das Patientenalter zum Operationszeitpunkt, der Body-Mass-Index oder die Zeitspanne zwischen auslösendem Ereignis und dem Operationszeitpunkt den Therapieeffekt beeinflussen.

Mit der Arbeitshypothese, dass ein signifikanter Unterschied zwischen den Skalenwerten der prä- und postoperativen Daten nachzuweisen ist, soll überprüft werden, ob durch die Knorpel-Knochen-Transplantation bei Vorliegen von Knorpelschäden im Grad III und IV nach Outerbridge auch langfristig positive Therapieeffekte zu erzielen sind.

Des Weiteren wird postuliert, dass ein erhöhter Body-Mass-Index, ein hohes Patientenalter, ein ausgeprägter Defekt, sowie eine lange Zeitspanne zwischen initialem Schmerzereignis und dem Operationszeitpunkt mit einem negativen Langzeittherapieeffekt korrelieren. 


\subsection{ANATOMIE UND FUNKTION DES KNIEGELENKES}

\subsubsection{Makroskopische Anatomie}

Das Kniegelenk als bikondyläres Drehscharniergelenk besteht aus den Anteilen von Femur, Tibia, Patella und den Gelenkanteilen der Menisken (Abb. 1). Das Kniegelenk ist ein zusammengesetztes Gelenk, welches aus dem Femoropatellargelenk und dem Femorotibialgelenk gebildet wird. Im Femorotibialgelenk artikulieren die Femurkondylen unter Einbeziehung der Menisken mit der tibialen Gelenkfläche. Die Inkongruenz von Femurkondylen und Tibiaplateau wird durch die Menisken ausgeglichen. Somit unterteilt sich das Femoropatellargelenk in das Meniskofemoral- bzw. Meniskotibialgelenk (Rauber und Kopsch 1987). Der anteriore Teil der femoralen Gelenkfläche (Trochlea mit medialer und lateraler Wange) bildet als Facies patellaris zugleich das patellare Gleitlager.
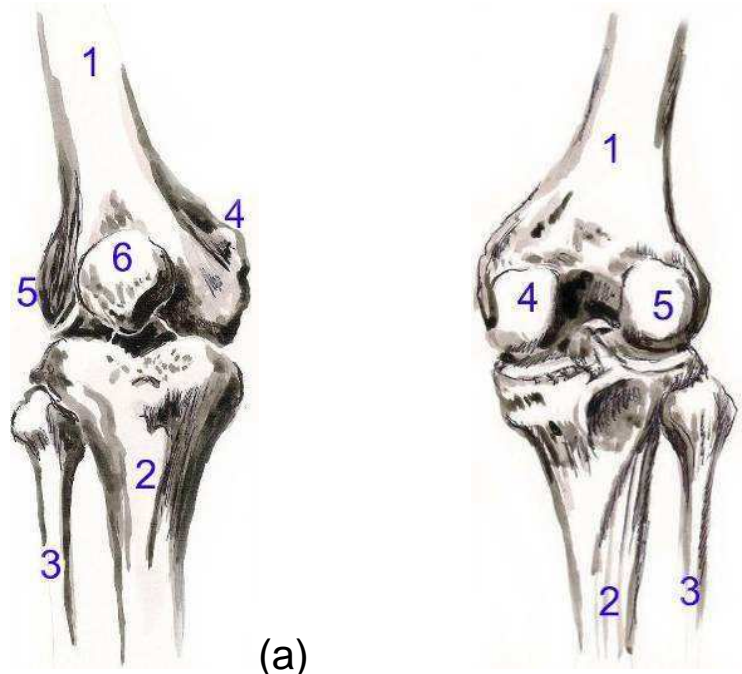

(a)

(b)

Abb. 1: $\quad$ Anatomie des Kniegelenkes I
(a) anterior, (b) posterior:
1 - Os femoris, 2 - Tibia, 3 - Fibula,
4 - Condylus medialis, 5 - Condylus lateralis 
Die Stabilität des Kniegelenkes wird durch Bänder und Sehnen inserierender Muskeln gewährleistet (Abb. 2). Die wichtigsten stabilisierenden Strukturen sind der M. quadriceps femoris und die Patella mit dem Lig. patellae sowie die Ligg. popliteum obliquum et arcuatum, der M. popliteus und Anteile des M. gastrocnemius. Lateral und ventral sind die Ligg. collaterale tibiale et. fibulare, die Sehnen des Pes anserinus, die Sehnen des M. semimembranosus und des M. biceps femoris, der Tractus iliotibialis sowie das Retinaculum patellae laterale an der Stabilisierung beteiligt. Intraartikulär sichern die Ligg. cruciata das Gelenk (Schiebler et al. 1995). Wichtige stabilisierende Funktion hat außerdem das Lig. collaterale obliquum (hinteres Schrägband). Es stellt den hinteren Teil des Lig. collaterale tibiale dar, welcher sich durch schräg verlaufenden Fasern vom Femur zum Meniscus medialis auszeichnet (Petersen et al. 2006).
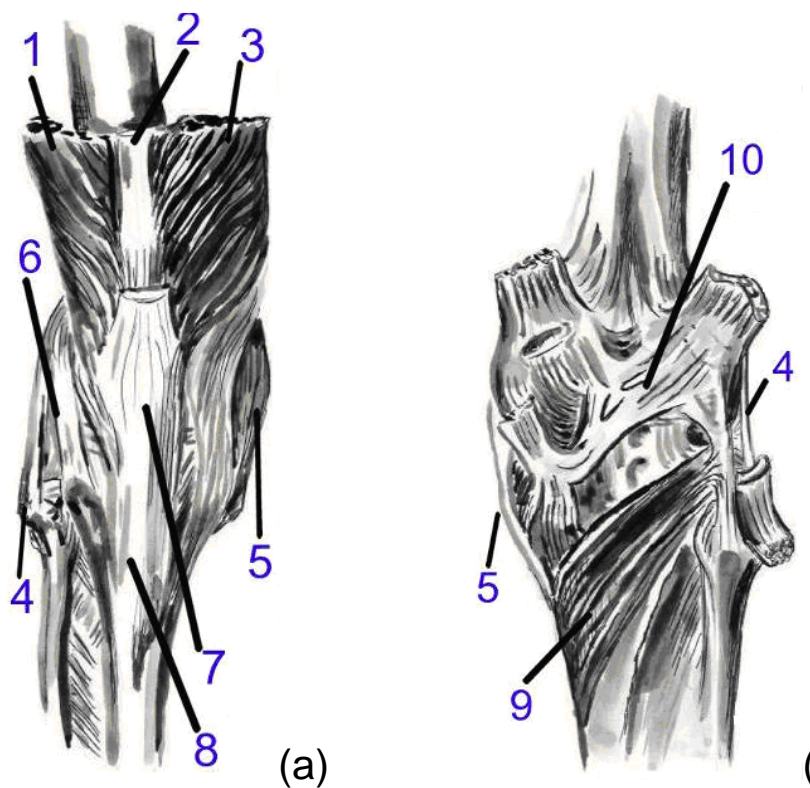

(b)

Abb. 2: $\quad$ Anatomie des Kniegelenkes II

(a) anterior, (b) posterior:

1 - M. vastus lateralis, 2 - Sehne M. rectus femoris,

3 - M. vastus medialis, 4 - Lig. Collaterale fibulare,

5 - Lig. collaterale tibiale, 6 -Tractus iliotibialis,

7 - Patella, 8 - Lig. patellae, 9 - M. popliteus,

10 - Lig. popliteum obliquum 


\subsubsection{Gelenkknorpel}

Die artikulierenden Anteile von Femur und Tibia besitzen einen Überzug aus hyalinem Knorpel, wobei die Dicke des Gelenkknorpels in Abhängigkeit von der Lokalisation variiert. Die von den Menisken bedeckten Bereiche des Tibiaplateaus sind mit einer dünneren Gelenkknorpelschicht ausgestattet, als die zentralen kraftaufnehmenden Bereiche (Kohn 2000). Die Schichtdicke des Knorpels ist beanspruchungsabhängig. Der Knorpel des Kniegelenkes hat normalerweise eine Schichtdicke von zwei bis vier Millimeter (Sellards et al. 2002). Retropatellar beträgt die Schichtdicke des Knorpels beispielsweise sieben bis acht Millimeter (Rauber und Kopsch 1987). Hyaliner Knorpel ist die häufigste Knorpelart des Menschen. Mikroskopisch stellt sich hyaliner Knorpel bläulich-weiß dar.

Der Knorpel setzt sich aus Zellen (Chondrozyten) und extrazellulärer Matrix zusammen, wobei die ECM von den Chondrozyten gebildet wird. Die Knorpelzellen werden von den Syntheseprodukten umschlossen und können dann nur durch Diffusion ernährt werden (Löffler und Petrides 2003). Bestandteile der extrazellulären Matrix sind Wasser (60 - $80 \%$ ), Kollagen Typ II (10 - $20 \%)$ und das Proteoglykan Aggrecan (5-7\%). Außerdem existieren in der ECM zu geringen Anteilen andere Proteoglycane und Kollagene (Typ V, VI, IX, X, XI). Des Weiteren kann man in der ECM Link-Protein, Anchorin, Hyaluronate, Fibronectin und Lipide finden (Martinek 2003).

Initial entwickelt sich Hyaliner Knorpel aus dem Mesenchym. Über Vorknorpelzellen differenzieren sich die Chondroblasten zu Chondrozyten (Rauber und Kopsch 1987). Schon in der fünften Embryonalwoche finden sich Knorpelbildungszentren. Aus dem mesenchymalen Umgebungsgewebe der Chondrozyten entsteht das Perichondrium als so genannte Knorpeldeckschicht (Geneser 1990). Dieses ist straffes kollagenes Bindegewebe und bedeckt, abgesehen von den Gelenkflächen, die Knorpelschicht (Rauber und Kopsch 1987). Einige Chondrozyten teilen sich und vermehren somit den Knorpel von innen heraus (interstitielles Knorpelwachstum). Durch appositionelles Wachstum differenzieren sich die umliegenden Mesenchymzellen zu Chondrozyten (Geneser 1990).

Die Haupteigenschaften des Knorpels liegen in der Aggregation von Hyaluronsäure (Elastizität) und den Proteoglykanen (Viskosität) begründet (Rauber und Kopsch 1987). Nach Junqueira et al. (2002) steht die Hyaluronsäure über Verbin- 
dungsproteine mit dem Zentralprotein des Proteoglykans in Verbindung (Abb. 3). Die größtenteils aus Chondroitinsulfat bestehenden Seitenketten binden an die Kollagenfibrillen. Proteoglykane werden unter dem Sammelbegriff Glykosamionoglykane (GAG) geführt. Als Bestandteil der ECM bestehen sie aus langen Heteroglykanketten und sich wiederholenden Dissaccharideinheiten. Sie können geordnete Strukturen ausbilden und als so genannte Polyanionen Moleküle reversibel binden. Dazu gehört vor allem Wasser. Wichtige Glykosamionoglykane der ECM des Knorpels sind Chondroitin-4-Sulfat und Keratansulfat (Löffler und Petrides 2003).

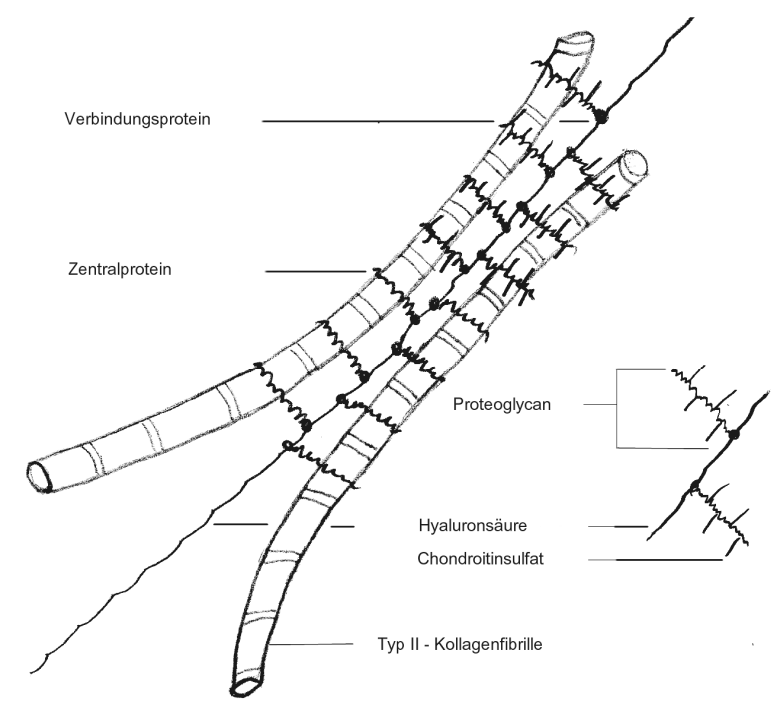

Abb. 3: $\quad$ Molekularer Aufbau der Knorpelmatrix (modifiziert nach Junqueira et al. 2002, S. 86)

1998 beschreiben Rudert und Wirth den zonalen Aufbau des Gelenkknorpels (Abb. 4a, S. 8). Es werden 4 Zonen unterschieden:

\begin{tabular}{|l|l|l|}
\hline Zone & Chondrozyten & ECM \\
\hline Tangentialfaserzone & $\begin{array}{l}\text { spindelförmig } \\
\text { angeordnet }\end{array}$ & $\begin{array}{l}\text { viel Kollagen, } \\
\text { wenig Proteoglykan }\end{array}$ \\
\hline Übergangszone & $\begin{array}{l}\text { in Chondromen, } \\
\text { abgerundet }\end{array}$ & $\begin{array}{l}\text { Konzentration der } \\
\text { Proteoglykane steigt }\end{array}$ \\
\hline Radiärzone & $\begin{array}{l}\text { Chondrozyten } \\
\text { säulenförmige } \\
\text { angeordnet }\end{array}$ & $\begin{array}{l}\text { Maximale Proteoglykankonzentration, } \\
\text { Wassergehalt am geringsten }\end{array}$ \\
\hline Zone des mineralisierten Knorpels & chondrozytenarm & Kalziumkristalle in die ECM eingelagert \\
\hline
\end{tabular}

Tab. 1: Zonen des Gelenkknorpels (nach Rudert und Wirth 1998) 
Basal geht die Zone des mineralisierten Knorpels (ZMK) in den subchondralen Knochen über (Rudert und Wirth 1998). Zwischen Radiärzone und der ZMK existiert die klassisch als basophil (HE-Färbung) und wellenförmig beschriebene Grenzlinie. Diese Grenzlinie (sog. „Tidemark“) ist trilaminär aufgebaut und trennt mineralisierten von nicht-mineralisiertem Knorpel. Sie stellt eine osteochondrale Verbindung dar, ist kollagenreich und enthält Hyaluron, jedoch fehlen die Glykosaminoglykane der Proteoglykane. Es wird vermutet, dass sie den hyalinen Knorpel vor passiver progressiver Mineralisation schützt (Lyons et al. 2005).

Mallinger et al. (1986) berichteten über die Anfärbbarkeit der GAG der extrazellulären Matrix des hyalinen Knorpels mit Alcian-Blau. Anhand dieser Färbung lässt sich der zonale Aufbau des hyalinen Knorpels nochmals veranschaulichen (Abb. $4 b)$.

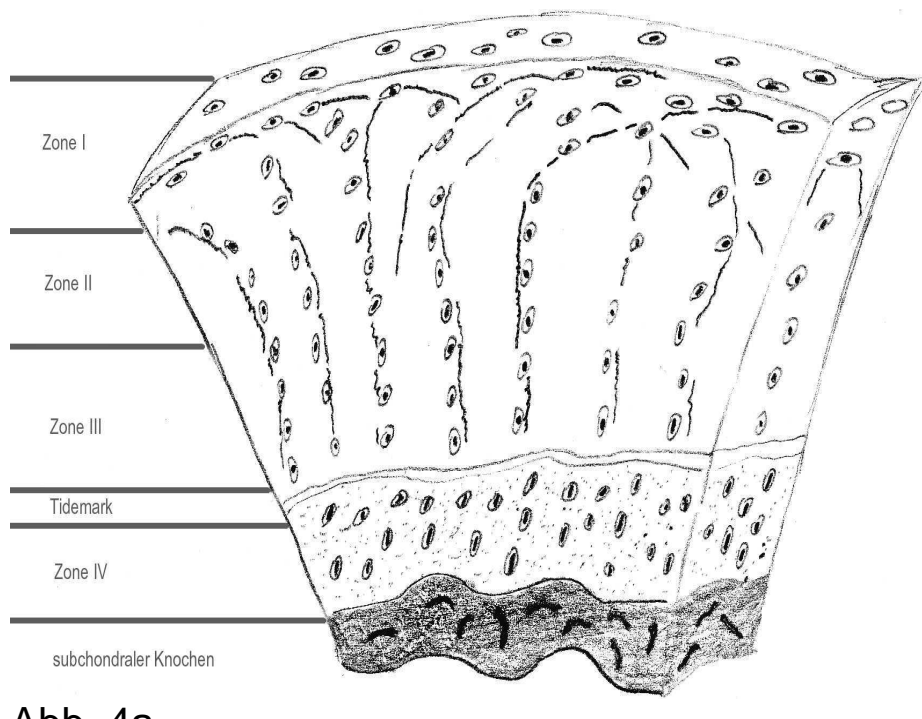

Abb. $4 a$

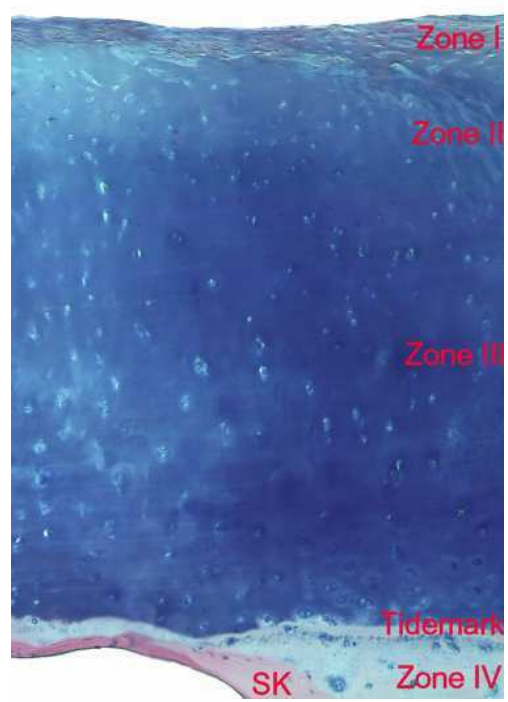

Abb. $4 b$

Abb. 4a: Zonen des Gelenkknorpels schematisch (modifiziert nach Rudert und Wirth 1998, S. 311): Zone I - Tangentialfaserzone, Zone II - Übergangszone, Zone III - Radiärzone, Zone IV - Zone des mineralisierten Knorpels

Abb. 4b: Zonen des gesunden Gelenkknorpels:

(Mikroskopische Darstellung des Gelenkknorpels nach Alcian-Blau-Färbung), SK - subchondraler Knochen

(Mit freundlicher Genehmigung von Hr. Prof. Dr. med. N. Miosge, AG orale Biologie und Geweberegeneration, Universitätsmedizin Göttingen.) 
Hyaliner Knorpel ist avaskulär, alymphatisch und aneural (Sellards et al. 2002). Obwohl die Chondrozyten nur 1 bis $10 \%$ des Knorpelvolumens ausmachen, gewährleisten sie jedoch die Synthese und den Abbau von Kollagenen und Proteoglykanen (Rudert und Wirth 1998). Für die Knorpelerhaltung und das Knorpelwachstum haben Ernährungsfaktoren und Hormone große Bedeutung (Geneser 1990). Die Ernährung des Knorpels erfolgt über die Synovialflüssigkeit und zu minimalen Anteilen über Gefäße des subchondralen Knochens (Rudert und Wirth 1998). An den Stellen, wo der Knorpel von Perichondrium umgeben ist, erfolgt die Ernährung über Diffusion aus dem perichondralen Gefäßnetz (Rauber und Kopsch 1987).

Hyalines Knorpelwebe wird hormonell vor allem durch Somatotropin beeinflusst. Über die Wirkung des Peptids Somatomedin wird die Zellproliferation und Sekretion der ECM gefördert. Synergistisch zum Somatotropin wirken die freien Schilddrüsenhormone, Testosteron, Vitamin C (Synthese der Kollagenfasern) und Vitamin D (Knorpelstoffwechsel und die Knorpelreifung). Östrogene und Cortison hemmen die Synthese von Chondroitinsulfat und Kollagen (Rauber und Kopsch 1987).

Statische Kompression inhibiert die Synthese der Moleküle in der ECM (Palmoski und Brandt 1984). Zyklisch dynamische Kompression stimuliert die Biosynthese (Sah et al.1998). Durch Kompression des Knorpels kommt es zu einer Deformierung der Zellen sowie der ECM (Gray et al. 1988). Dadurch wird sowohl die Elektronenkonzentration der ECM als auch pH-Wert und Osmolarität verändert. Eine Belastung führt zum Flüssigkeitsaustausch (Bhakta et al. 2000). Folglich resultiert ein Nährstoff- und Molekültransport. Die Regenerationsfähigkeit des Knorpelgewebes aus dem Perichondrium endet mit dem Eintreten des Erwachsenenalters (Geneser 1990). 


\subsubsection{Biomechanik}

Das Femoropatellargelenk und das Femorotibialgelenk bilden eine gekoppelte mechanische Einheit im Sinne eines Drehscharniergelenkes mit 2 Freiheitsgraden. Auf das Femorotibialgelenk wirkt beim Einbeinstand die Gelenkresultierende, bestehend aus der Vektorsumme von Körpergewicht abzüglich Unterschenkel-FußGewicht des Standbeines und der Traktusspannung (Kraftsumme von Tractus iliotibialis und Musculus tensor fasciae latae). Auf das Tibiaplateau wirkt bei einbeiniger Belastung das Doppelte des Körpergewichtes. Durch die zusätzlich auftretenden dynamischen Kräfte beim Gehen kann die Belastung auf das Sechsfache des Körpergewichtes gesteigert werden.

Das Femoropatellargelenk wird aufgrund inserierender Bänder und Muskeln insbesondere durch vertikale und horizontale Zugkräfte belastet. Die vertikalen Kräfte nehmen dabei in Beugestellung zu. Die kraftaufnehmende Fläche im Femoropatellargelenk hängt vom Beugegrad im Kniegelenk ab und ist bei ca. $60^{\circ}$ Beugung des Kniegelenkes am größten. Mit zunehmender Beugung wird die Patella im femoralen Gleitlager angepresst (Rauber und Kopsch 1987).

Der Knorpel spielt eine wesentliche Rolle bei der Kraftübertragung (Druck) im Kniegelenk und ist entscheidend für die Gleitfunktion während der Bewegung des Gelenkes. Die Druckfestigkeit ist höher als die Zugfestigkeit (Rauber und Kopsch 1987). Die Druckbelastung kann bis zu $400 \mathrm{Kg} / \mathrm{cm}^{2}$ betragen (Puhl 1997). Vergleicht man beispielsweise die Reibungskoeffizienten von hyalinem Gelenkknorpel $(0,2$ - 0,002) mit dem von Stahl auf Stahl $(0,6)$, so ist der Reibungskoeffizient des Knorpels deutlich geringer (Mow et al. 1984).

Spahn und Wittig stellen 2003 biomechanische Untersuchungen zum Spannungsund Bruchverhalten des gesunden Gelenkknorpels unter axialer Belastung an. Hier zeigt sich, dass hyaliner Knorpel mit einem Elastizitätsmodul von ca. $40 \mathrm{MPa}$ weich ist. Bis zu einer Druckbelastung von $6 \mathrm{MPa}$ ist hyaliner Knorpel elastisch. Bei Werten über $6 \mathrm{MPa}$ kommt es zur plastischen Verformung. Die durchschnittliche Bruchspannung des hyalinen Knorpels von 25,8 MPa entspricht nach Spahn und Wittig 2003 der Kraftentwicklung beim ungebremsten Aufprall aus 4,3 m Höhe. 


\subsection{PATHOLOGIE DES KNORPELS}

\subsubsection{Epidemiologie des Knorpelschadens}

Die Epidemiologie von Knorpelschäden ist heute gut untersucht (Curl et al. 1997, Hjelle et al. 2002, Årøen et al. 2004, Figueroa et al. 2007, Widuchowski et al. 2007). Bei Patienten mit Kniegelenkbeschwerden wurde arthroskopisch der Knorpelstatus beurteilt. Dazu wurden Daten zu Prävalenz, Durchschnittsalter, Schädigungsgrad (nach Outerbridge oder ICRS), Lokalisation und Begleitpathologie erhoben (Tab. 2). Demnach bestehen bei Patienten mit Kniegelenkbeschwerden größtenteils in ca.

60 \% Knorpelschäden. Die Prädilektionsstellen sind der mediale Femurkondylus bzw. die Patella. Den Hauptanteil bilden die III.- und IV.gradigen Knorpelschäden. Die durchschnittliche Defektgröße wird mit $2 \mathrm{~cm}^{2}$ angegebenen (Hjelle et al. 2002 und Figueroa et al. 2007). Insbesondere Läsionen des medialen Meniskus sowie des vorderen Kreuzbandes sind die häufigsten Begleitbefunde. Nach Untersuchungen von Årøen et al. (2004) standen Sport und körperliche Betätigung häufig im Zusammenhang mit einem Knorpelschaden. Insbesondere Kontaktsportarten (Fußund Handball) standen dabei im Vordergrund.

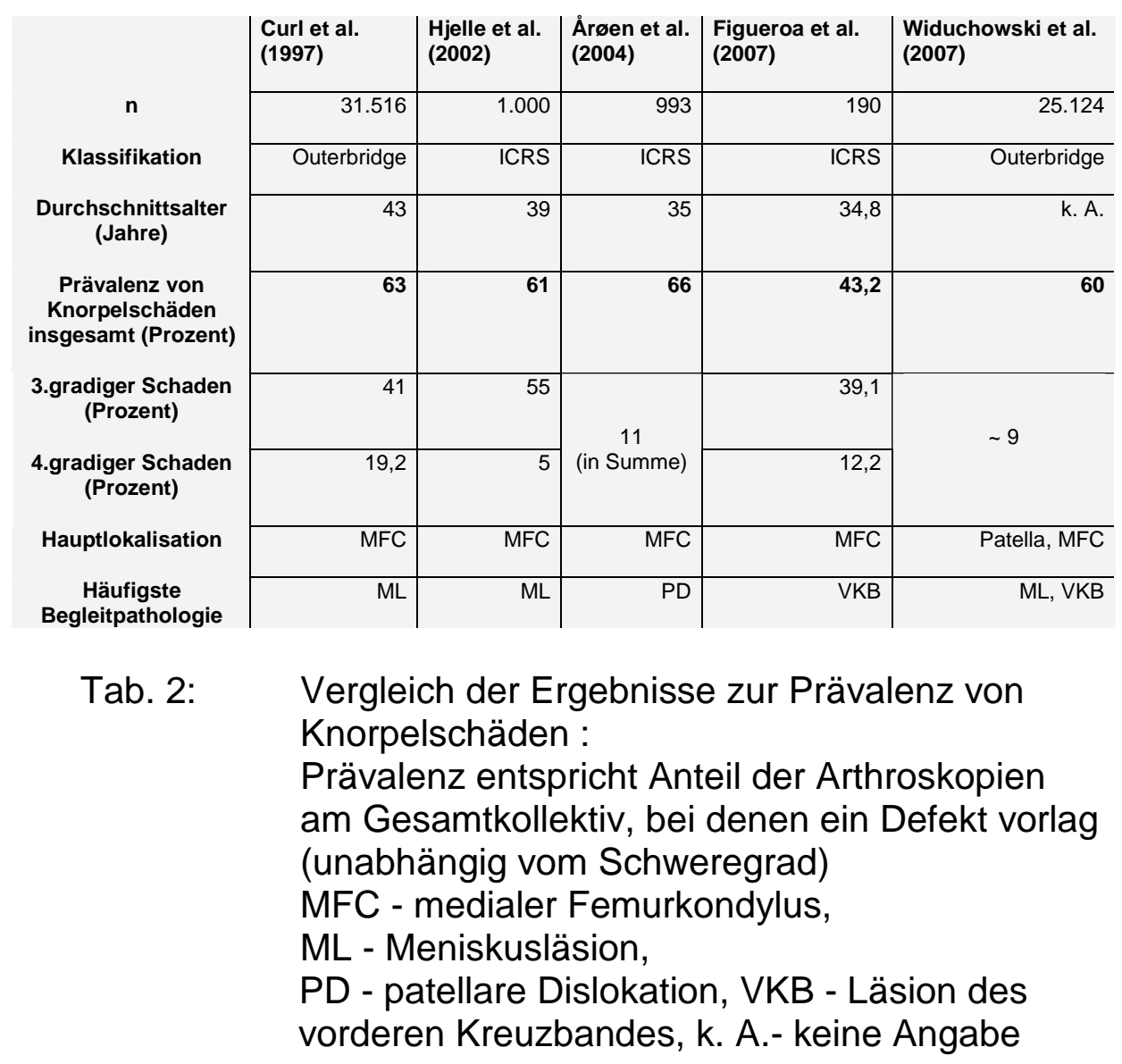




\subsection{2 Ätiologie des Knorpelschadens}

Eine Schädigung der Knorpelstruktur kann aus Makro- oder rezidivierenden Mikrotraumen resultieren (Buckwalter 1995). Diese Knorpelverletzung ist als ätiologischer Faktor in der Entstehung des abnutzungsbedingten Knorpelschadens $\mathrm{zu}$ werten. Voneinander abzugrenzen sind der traumatische Knorpelschaden (Verletzung) und die manifeste Arthrose (Abnutzung) (Hempfling und Weise 2007).

\subsubsection{Pathomechanik und Pathogenese}

Der Unfallmechanismus muss beim Vorliegen einer Knorpelverletzung differenziert betrachtet werden. Beim indirekten Trauma, hervorgerufen beispielsweise durch Bandläsionen, treten insbesondere tangentiale Scherkräfte auf. Hierbei besteht die Möglichkeit eines Knorpelschadens mit Beteiligung des subchondralen Knochens. Knorpelverletzungen sind oft Begleitschäden und werden durch indirekte oder direkte Kräfte hervorgerufen. Isolierte Knorpelverletzungen sind selten. Teilweise kann das Verletzungsmuster auf die Knorpelverletzung hinweisend sein (Hempfling und Weise 2007).

Bei Vorliegen eines traumatischen Knorpelschadens beeinflusst das Ausmaß des Traumas sowie die Menge der einwirkenden Kraft die resultierende Tiefe des Schadens bzw. das Ausmaß der Knorpelschädigung. Geringe Traumen zeigen oberflächliche Schäden im Sinne einer Zell- und Matrixschädigung. Sichtbare Schädigungen der Oberfläche liegen dabei nicht vor. Aus dem Boden größerer Traumen entstehen sichtbare chondrale Fissuren oder ein Höhenverlust der Knorpelsubstanz. Die maximale Schädigung zeigt sich in einer osteochondralen Fraktur. Hier resultiert die Schädigung der kompletten Knorpelsubstanz aus der Schädigung des subchondralen Knochens (Alford und Cole 2005). Da hyaliner Gelenkknorpel elastischer als der Knochen ist, kommt es zunächst zu einer Schädigung subchondraler Strukturen, bevor eine Knorpelschädigung entsteht (Lahm 2002).

Das pathomorphologische Substrat des abnutzungsbedingten Knorpelschadens muss von dem der Knorpelverletzung abgegrenzt werden. Während sich ein Knorpelschaden über eine Knorpelerweichung (Malazie Grad I-III) zur Arthrose 
entwickelt, wird bei der Knorpelverletzung das in Tabelle 3 dargestellte pathomorphologische Substrat beschrieben (Hempfling und Weise 2007).

\begin{tabular}{|c|c|}
\hline Direkte Krafteinwirkung & $\begin{array}{l}\text { Grad 0-elastoide Verformung } \\
\text { Grad 1-trabekuläre Fraktur } \\
\text { Grad 2-subchondrale Impression } \\
\text { Grad 3-Impressionsfraktur } \\
\text { Grad 4-osteochondrale Impression }\end{array}$ \\
\hline Scherkraft & $\begin{array}{l}\text { Grad 0-elastoide Verformung } \\
\text { Grad 1-chondrale Fissur } \\
\text { Grad 2-Fissur bis subchondrale } \\
\text { Grad 3-Knorpelfraktur } \\
\text { Grad 4-osteochondrale Impression }\end{array}$ \\
\hline
\end{tabular}

Tab. 3: $\quad$ Pathomorphologisches Substrat der Knorpelverletzung (Hempfling und Weise 2007)

Im pathogenetischen Ablauf der Knorpelverletzung und der folgenden Veränderungen wird dem Initialschaden mit Eröffnung der Tangentialfaserschicht eine wesentliche Bedeutung zugeschrieben (Buckwalter und Mankin 1998). Die Knorpelschicht weist dann einen erhöhten Flüssigkeitsgehalt auf. Die Kollagenfasern in der Tangentialfaserschicht werden auseinandergedrückt und verlieren die Belastbarkeit. Proteoglykane gehen verloren. Die oberflächlich liegenden Knorpelzellen sterben ab. Am Defektrand werden Chondrozytenansammlungen gebildet. Die Stoffwechselaktivität der Chondrozyten ist erhöht und folglich die Grundsubstanzproduktion gesteigert. Es resultiert jedoch nur eine Reparatur und keine Regeneration. Bei Fortschreiten des Defektes in bis auf die Basalschicht kommt es zur Zerstörung der Kollagenstruktur, zu einer entzündlichen Begleitreaktionen und durch den anfallenden Detritus zur weiteren Zerstörung und Funktionseinbuße. Bestehen größere Defekte kann auch durch den Reparatureffekt keine Kompensation erreicht werden und es resultiert ein Dauerschaden (Buckwalter und Mankin 1998). Die Eröffnung des subchondralen Knochens ist ein entscheidender Prozess hinsichtlich der intrinsischen Reparationsfähigkeit. 
Neben der Steigerung der chondrozytären Stoffwechselaktivität kann sich hier der Defekt mit Blutkoageln füllen, mesenchymale Zellen können einwandern und fibröser Knorpel kann gebildet werden (Buckwalter und Mankin 1998).

Durch das entstandene Ersatzgewebe kann jedoch keine vollständige Funktionsübernahme gewährleistet werden (Mitchell und Shepard 1976).

\subsubsection{Klinik}

Gelenkknorpel ist anerval (Sellards et al. 2002). Daher bereitet der Knorpelschaden an sich keine Schmerzen. Einerseits erklärt man sich den Schmerz durch eine begleitende Synavialitis (Bethge 1962). Andererseits geht man von einer Schmerzquelle im subchondralen Knochen (nozizeptive Fasern des Knochenmarks) aus (Hempfling und Weise 2007). Die klinische Symptomatik ist weitestgehend abhängig von Ausmaß der Knorpelschädigung bzw. der zusätzlich betroffenen Strukturen. Dementsprechend können Schmerzen, Gelenkinstabilitäten (bei Vorliegen einer Schädigung des Kapsel-Band-Apparates), ein Kniegelenkerguss, Einschränkungen des Bewegungsausmaßes der Extremität (z.B. bei Vorliegen eines freien Gelenkkörpers) und ein Entlastungshinken resultieren. Es ist untersucht, dass beispielsweise Rupturen des vorderen Kreuzbandes in 20 - 40 \% mit sichtbaren Knorpelverletzungen einhergehen (Coen et al. 1996). 


\subsection{DIAGNOSTIK}

\subsubsection{Anamnese und Klinische Untersuchung}

Den ersten Schritt in der Diagnostik stellt die exakte Anamneseerhebung dar. Hierbei spielen der Unfallmechanismus, der Zeitpunkt des Auftretens der ersten Symptome sowie die Symptomkonstellation eine entscheidende Rolle. Des Weiteren ist es von Bedeutung, Daten hinsichtlich Voroperationen und deren Ergebnisse zu erheben. Dazu ist es sinnvoll, Dokumente wie Operationsberichte, Bilder und Videos einzusehen. Zusätzlich kann im Falle mehrerer Voroperationen und der Behandlung durch verschiedene Operateure der direkte Kontakt hilfreich sein (Alford und Cole 2005).

Zunächst ist bei der Erhebung des klinischen Befundes auf das Vorliegen eines Gelenkergusses, Krepitationen, Unfallzeichen wie Prellmarken, Hämatome und Hautläsionen zu achten und das Bewegungsausmaß der Extremität zu bestimmen (Zacher 2006). In der klinischen Untersuchung ist die Untersuchung auf Begleitpathologien obligat. Dazu zählt insbesondere die Beurteilung des Kapsel-Band-Apparates, des Patellalaufs, der Beinachse sowie der Menisken. Dies ermöglicht das Ein- bzw. Ausschließen von Patienten in ein bestimmtes Behandlungsschema mit dem entsprechenden operativen Eingriff und postoperativem Rehabilitationsprotokoll (Alford und Cole 2005).

\subsubsection{Konventionelles Röntgen}

Im Rahmen der konventionell-radiologischen Diagnostik sind folgende Röntgenaufnahmen des Kniegelenkes obligat: a. p., seitlich, Patella tangential, a. p. in 30 Grad Flexion unter Belastung (Böttner 2008). Des Weiteren ist eine Ganzbeinaufnahme zur Achsbestimmung notwendig (Alford und Cole 2005). Eine direkte Beurteilung des Knorpels bzw. der Knorpelverletzung kann dadurch jedoch nicht erzielt werden. Pathologien zeigen sich erst bei höhergradigen Schäden der Gelenkflächen. Hier korreliert der Grad der Schädigung mit der Reduktion der Gelenkspaltweite. Bei Knorpelschäden im Grad I und II nach Outerbridge zeigen die Röntgenbilder meist einen Normalbefund (Spahn et al. 2007). 


\subsubsection{Magnetresonanztomographie}

In der heute hochauflösenden MRT ist die Darstellung des Knorpels sowie die 3-DAusmessung der Knorpeldicke nicht-invasiv durchführbar. Am häufigsten werden die Fast-Spin-Echo-Sequenz und die dreidimensionale, fettunterdrückte oder wasserangeregte Gradientenechosequenz angewandt (Trattning et al. 2004). Als Hauptproblem in der derzeitigen magnetresonanztomographischen Beurteilung gelten die nicht standardisierten Untersuchungstechniken (Potter et al. 1998). Für eine effektive Bildgebung der Strukturen am Kniegelenk mit hochauflösenden Geräten (mindestens größer $1 \mathrm{~T}$ ) wird eine Raumdimension 0,3 bis 0,5 Voxel sowie eine Schichtdicke von 3 bis $4 \mathrm{~mm}$ benötigt. Zur magnetresonanztomographischen Beurteilung der Knorpelstruktur wird daher von der ICRS ein spezielles Untersuchungsprotokoll (Abb. 5) empfohlen (Brittberg und Winalski 2003).

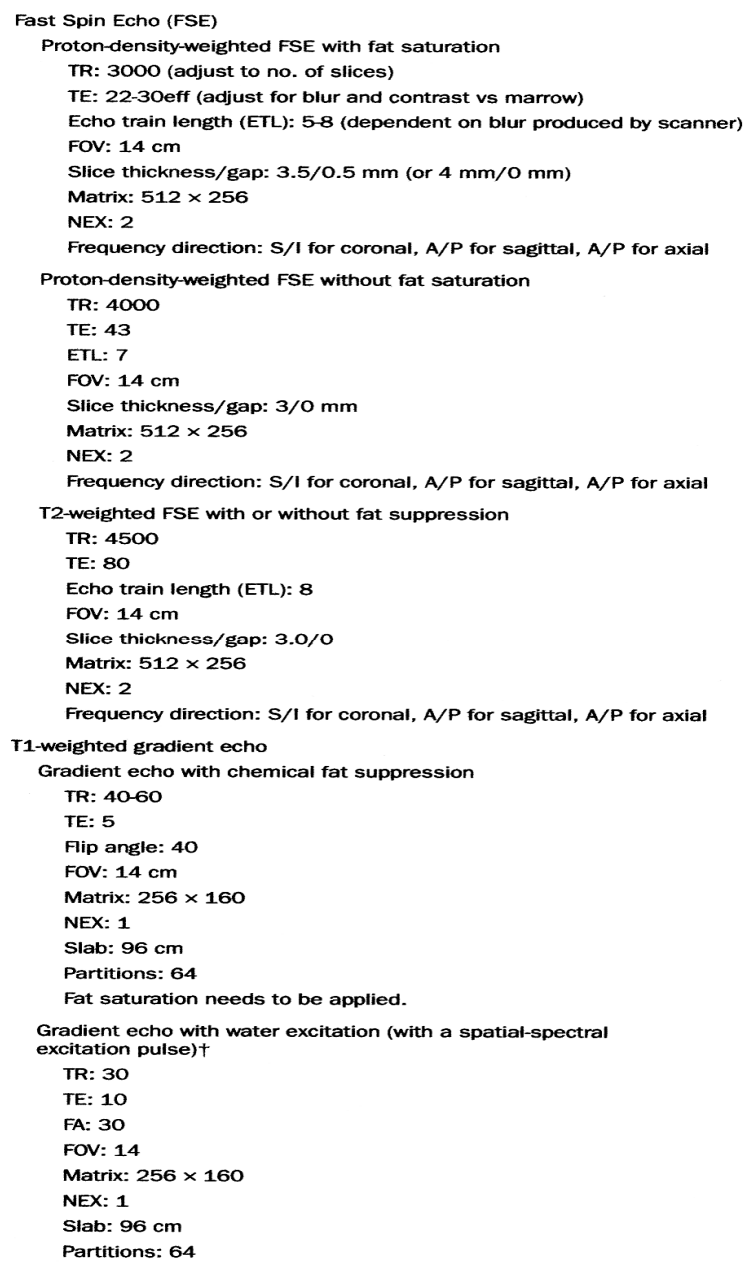

Abb. 5: $\quad$ MRT-Studienprotokoll (Brittberg und Winalski 2003) (Mit freundlicher Genehmigung der ICRS) 
Der Schweregrad des Knorpelschadens wird in der MRT anhand der Klassifikation von Vallotton (Vallotton et al. 1995) beurteilt (Tab. 4).

\begin{tabular}{|l|l|}
\hline Grad I & Lokale Hyper- bzw. Hypodensität oder oberflächliche Unregelmäßigkeiten \\
\hline Grad II & $\begin{array}{l}\text { Partieller Defekt mit Reduktion der Knorpeldicke unter 50\%, der jedoch nicht } \\
\text { bis auf den subchondralen Knochen reicht }\end{array}$ \\
\hline Grad III & $\begin{array}{l}\text { Tiefer Defekt mit Reduktion der Knorpeldicke um mehr als 50\%, der bis auf } \\
\text { den subchondralen Knochen reicht }\end{array}$ \\
\hline Grad IV & Kompletter Defekt mit subchondraler Sklerose bzw. subchondralen Zysten \\
\hline
\end{tabular}

Tab. 4: $\quad$ MRT-Klassifikation des Knorpelschadens (Vallotton et al. 1995)

Die fett-unterdrückenden Sequenzen ermöglichen die Darstellung des subchondralen Ödems (sogenannter „Bone-Bruise“) (Böttner 2008). Dieses kann nur mittels MRT diagnostiziert werden und ist ein wichtiger Parameter für die Abschätzung der akuten Verletzung (Abb. 6). Es ist jedoch wenig spezifisch, da es beispielsweise auch als Begleiterscheinung bei Tumorerkrankungen, Entzündungen und Arthrose vorliegen kann. Hinsichtlich der Diagnose einer akuten Knorpelschädigung gibt es beweisende Kriterien. Dazu zählen zum einen der Nachweis eines pathologischen Knorpelbefundes unter Vorliegen eines subchondralen Ödems innerhalb der ersten 6 Wochen nach stattgehabtem Trauma, sowie Verringerung des Ödems im Verlauf unter Ausschluss arthrotischer Veränderungen. Zum anderen gilt ein subchondrales Ödem ohne Vorliegen einer initialen Knorpelpathologie mit nachweisbarer Verringerung des Ödems in der Verlaufsuntersuchung nach drei Monaten unter Neuauftreten eines Knorpelschadens als beweisend (Hempfling und Weise 2007).

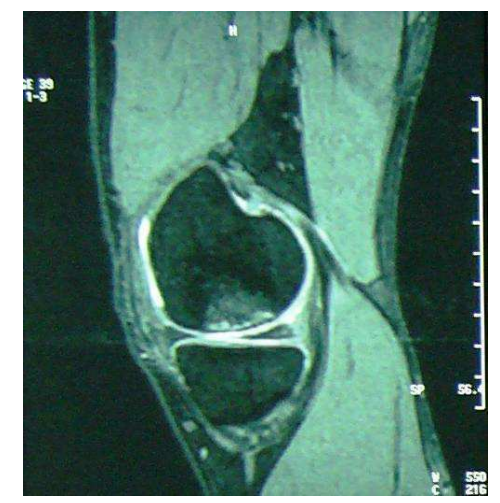

Abb. 6: $\quad$ MRT des rechten Kniegelenkes mit sog. „Bone-Bruise“ 
Der initiale Knorpelschaden auf chondrozytärer Ebene kann derzeit in der MRT nicht erfasst werden. Erst mit zunehmendem Schädigungsgrad erhöht sich die Aussagekraft der MRT (Hempfling und Weise 2007). Insbesondere Knorpelschäden im Grad III und IV nach Outerbridge können mit der MRT gut beurteilt werden. Arthroskopieund MRT-Befund korrelieren signifikant bei Vorliegen von höhergradigen Schäden im Sinne von Grad III und IV nach Outerbridge. Normaler Knorpel und Knorpelschäden im Stadium I und II nach Outerbridge werden in der MRT signifikant überbewertet. Dies erklärt man sich anhand der Abhängigkeit der Knorpelsignalintensität von der Ausrichtung der Kollagenfasern vom Magnetfeld. Beim Vorliegen von Strukturveränderungen in den Kollagenfaserausrichtungen kann dies pathologische Zustände vortäuschen (Spahn et al. 2007).

Durch die MRT ist es des Weiteren möglich, postoperativ Aussagen über die Knorpel-Knochen-Struktur zu erhalten. Nicht-invasiv können beispielsweise nach stattgehabter Knorpel-Knochen-Transplantation die Knorpel-Knochen-Zylinder lokalisiert und deren Beziehung zum umliegenden Gewebe (Knorpel, Knochen) beurteilt und Komplikationen erkannt werden (Bobic 1996). Des Weiteren ist es möglich, die Rückbildung des subchondralen Knochenödems im Verlauf zu beurteilen (Trattnig 2004). Bei Persistenz des subchondralen Ödems ist hier von einer schlechten Einheilung auszugehen. Kongruenz von Zylinder und Gelenkfläche geben Aufschluss über Implantationsprobleme bzw. eine Zylinderdegeneration. Aus einer Inkongruenz resultiert eine verstärkte Transplantatbelastung und -destruktion (Dew und Martin 1992).

\subsubsection{Arthroskopie}

Wenn die Knorpeloberfläche betroffen ist, ist die Arthroskopie derzeit das aussagkräftigste Verfahren in der Beurteilung des Knorpelschadens. Es existieren verschiedene Klassifikationen (Outerbridge 1961, Ficat und Hungerford 1977, Metcalf 1982, Hille 1988, Bauer und Jackson 1988), welche sich entweder an der Schadensausdehnung oder dem Erscheinungsbild orientieren (Hempfling und Weise 2007).

Zur arthroskopischen Beurteilung von Knorpelschäden ist die Klassifikation nach Outerbridge (Outerbridge 1961) ein gängiges Verfahren (Tab. 5, S. 19). Ausgehend vom Normalbefund (Abb. 7, S. 19) reichend bis zum Vollschicht-Knorpelverlust (Grad IV, Abb. 9, S. 19) wird der intraoperative, jedoch subjektive und untersucherabhängige Befund erhoben (Spahn 2007). 


\begin{tabular}{|l|l|}
\hline Grad 0 & Normalbefund, intakter Knorpel mit glatter, weißer Oberfläche und Konsistenz (Abb. 7) \\
\hline Grad I & Erweichung der Oberfläche, aber glatt erhaltene Oberfläche ohne Fibrillationen \\
\hline Grad II & Oberfläche aufgefasert mit Einrissen und deutlicher Fibrillation (Abb. 8) \\
\hline Grad III & $\begin{array}{l}\text { Tiefe Fissuren, Ulcus bzw. Krater mit instabilen und unterminierten Rändern, } \\
\text { subchondraler Knochen noch nicht erreicht }\end{array}$ \\
\hline Grade IV & Vollschicht-Knorpelverlust bis auf den subchondralen Knochen (Abb. 9) \\
\hline
\end{tabular}

Tab. 5: $\quad$ Klassifikation des Knorpelschadens nach Outerbridge (Outerbridge 1961)

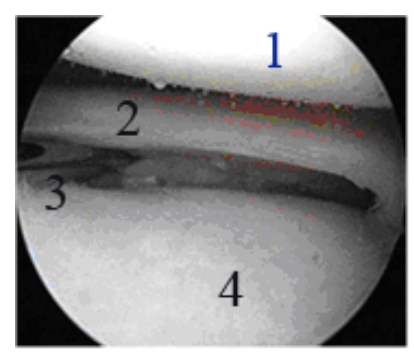

Abb. 7: $\quad$ Arthroskopischer Normalbefund (lateraler Gelenkspalt mit Teilen des Außenmeniskus) 1 - Femurkondylus, 2 - Außenmeniskus,

3 - M. popliteus, 4 - Tibiaplateau

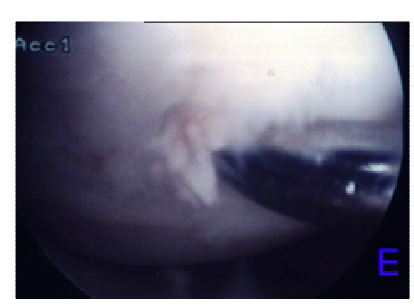

Abb. 8: Knorpelschaden Grad II nach Outerbridge (Oberfläche aufgefasert mit Einrissen und deutlicher Fibrillation)

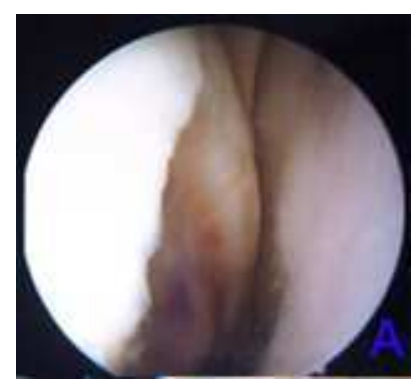

Abb. 9: Knorpelschaden Grad IV nach Outerbridge (Vollschicht-Knorpelverlust bis auf den subchondralen Knochen) 
Die arthroskopische Operation ermöglicht die Entfernung von Fragmenten, Glättung von Kanten und die Untersuchung der Läsion mit dem Tasthaken. Insbesondere ist es möglich, zur Detektion der Defekttiefe jede Fissur zu verfolgen (Brittberg und Winalski 2003). Zur Beschreibung des Defektes nach Debridement existiert die von der ICRS beschriebene Klassifikation (Abb. 10). In diese Klassifikation gehen Defekttiefe (Grad 0 bis 4) sowie die Beschreibung des Defektareals (normal bis stark abnormal) ein.
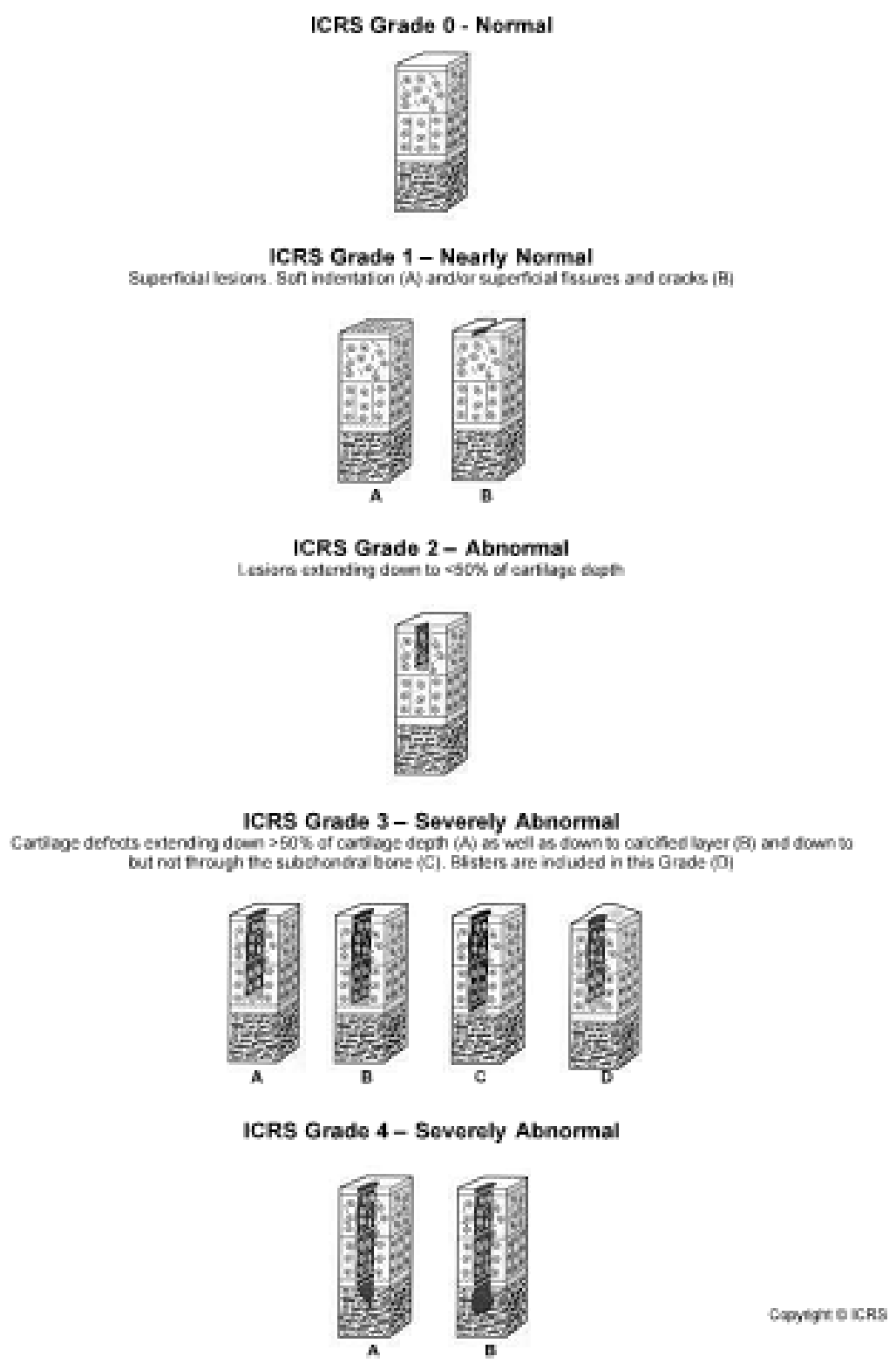

Abb. 10: Klassifikation des Knorpelschadens nach ICRS (Brittberg und Winalski 2003)

(Mit freundlicher Genehmigung der ICRS - International Cartilage Repair Society) 


\subsection{THERAPIE}

\subsection{1 konservative Therapie}

Konservative Therapiemaßnahmen stellen bei Knorpelschäden keine ursächliche Therapie dar. Sie dienen in erster Linie der Schmerzreduktion, der Verbesserung der Gelenkfunktion, des Bewegungsausmaßes sowie der Verbesserung der Gelenkstabilität. Dazu zählen auch allgemeine Maßnahmen wie Gewichtsreduktion (Gewichtsreduktion um $5 \mathrm{Kg}$ verringert die symptomatischen Beschwerden um 50 \% (Fritz et al. 2003)) und eine adaptierte Belastung (bessere Ernährung des Knorpels). Die physikalische Therapie in Form der Kryotherapie zur Detonisierung der Muskulatur, in Form von Wärmetherapie zur Förderung der Dehnbarkeit des Bindegewebes sowie die Elektrotherapie zur Durchblutungsförderung und Analgesie spielen eine wesentliche Rolle. Der Ultraschall (mechanische Mikrovibration) und die pulsierenden elektromagnetischen Felder (Durchblutungsförderung, Regeneration, Aktivierung des Immunsystems) sind weitere konservative Therapiemöglichkeiten. Zusätzlich umfasst das weitere konservative Behandlungskonzept physiotherapeutische Maßnahmen sowie die orthopädietechnische Versorgung mit dämpfendem Schuhwerk, einer Schuhranderhöhung oder der Anpassung von Orthesen und Gehstützen.

Die medikamentösen Behandlungsmöglichkeiten von Knorpelschäden sind begrenzt. Es kann nur eine symptomatische Therapie erzielt werden. Diese medikamentöse Therapie fokussiert hauptsächlich die Schmerzlinderung (Analgetika, Opiate, NSAR, Flupirtin) und Entzündungshemmung (NSAR, Glukokortikoide). Alternative medikamentöse Therapiemöglichkeiten stellen die SADOA (,slow acting drugs in osteoarthritis“) dar. Diese wirken symptomatisch (D-Glucosaminsulfat und Hyaluronsäure) oder modifizieren den Gelenkstatus (DMOAD, wie IL-1-Rezeptorantagonisten, Orthokin® und Anakinra) und verlangsamen zumindest den Progress einer Knorpelschädigung (Fritz et al. 2003). 


\subsection{2 operative Therapie}

Zur operativen Behandlung von Knorpelverletzungen liegen stadiengerechte Therapieansätze vor (Braun et al. 2007).

Bei Defekten im Stadium III und IV nach Outerbridge rücken die Abrasionsarthroplasik (Johnson 1986), die Mikrofrakturierung (Steadman et al. 1997) und die Anbohrung nach Pridie (Pridie 1959) in den Hintergrund, da es hier ausschließlich zur Bildung von Ersatzgewebe kommt, welches sich deutlich in seiner Funktion von hyalinem Knorpel unterscheidet (Buckwalter und Lohmander 1994). Operative Maßnahmen zur Sanierung eines Defektes im Stadium III und IV nach Outerbridge stellen die autologe Chondrozytentransplantation ( $\mathrm{ACl}$ oder $\mathrm{MACl}$ ) und die in dieser Studie angewandte Knorpel-Knochen-Transplantation unter Verwendung von Autografts (OATS, Mega-OATS) dar (Imhoff et al. 2006). Des Weiteren besteht die Möglichkeit der Implantation von osteochondralen Allografts (Bugbee und Convery 1999).

\subsubsection{Regenerative Strategien}

Zu den regenerativen Strategien zählen die Abrasionsarthroplastik (Johnson 1986), die Anbohrung nach Pridie (Pridie 1959) sowie die Mikrofrakturierung (Steadman et al. 1997). Im Rahmen der Pridie-Bohrung wird das denudierte Knorpelareal mit Kirschner-Drähten angebohrt (Abb. 11).

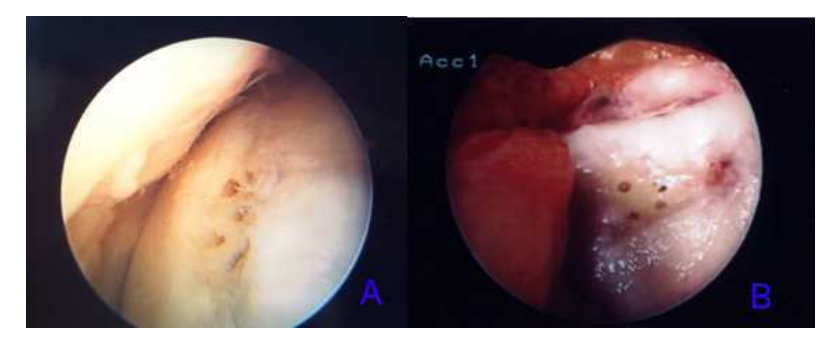

Abb. 11: $\quad$ Arthroskopischer Befund nach Pridie-Bohrung

Bei der Abrasionsarthroplasik werden 1 - 3 Millimeter des subchondralen Knochens mit einem Bohrer entfernt. Steadman entwickelte 1997 die o. g. Techniken weiter, indem er mit einem Pfriem den subchondralen Knochen perforiert, somit die die thermische Zellnekrose reduzierte und multipotente Knochenmarkstammzellen zum 
Einströmen anregte (Steadman et al. 1997). Obligate ist eine Perforationstiefe des subchondralen Knochens, bis es sichtbar zum Einströmen von Fettropfen kommt (Steadman et al. 2001). Durch die Anwendung der o. g. Techniken wird aufgrund der Differenzierung mesenchymaler Stammzellen eine Bildung von Reparaturgewebe (Faserknorpel) induziert (Rolauffs et al. 2009). Dieser zeigt jedoch keine optimalen biochemischen, biomechanischen und Verschleißeigenschaften (Buckwalter et al. 1994).

\subsubsection{Gewebeersatz}

Zum direkten Gewebesatz werden osteochondrale Allografts (Shasha et al. 2003) und osteochondrale Autografts (Hangody et al. 2004) benutzt. Die Knorpel-KnochenTransplantation bzw. Mosaikplastik wurde erstmals 1985 von Yamashita (Yamashita et al. 1985) angewandt. Es werden aus gesunden und unbelasteten Zonen Zylinder entnommen (hyaliner Knorpel mit subchondralem Knochen) und in vorgestanzte Löcher des ehemals zerstörten und debridierten Areals „press-fit“ eingebracht (Rolauffs et al. 2009). Initial wird die Präparation des Defektes durchgeführt. Durch Anfrischen der Ränder und Ausschabung bis auf den subchondralen Knochen wird der Defekt für die Transplantataufnahme vorbereitet und ausgemessen (Durchmesser und Defekttiefe). Anschließend wird die Transplantatanzahl und die Transplantatgröße bestimmt. Dann erfolgen nach Lokalisierung der Entnahmestelle die Entnahme des Transplantates und die Implantation. Dazu wird zunächst entsprechend vorgebohrt, dann dilatiert und letztlich das Transplantat "press-fit“ eingebracht (Hangody et al. 2004, Abb. 12).

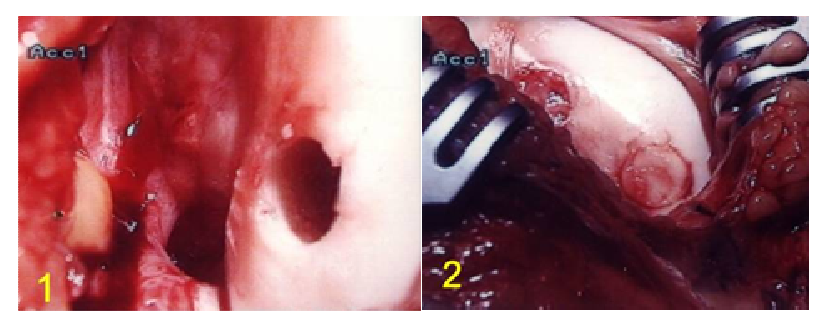

Abb. 12: OP-Situs am medialen Femurkondylus

1 - Entnahmestelle des Zylinders

(Länge $2 \mathrm{~cm}$, Durchmesser $10 \mathrm{~mm}$ )

2 - eingebrachtes Transplantat 
Nachteile der Knorpel-Knochen-Transplantation sind das Hervorrufen einer neuen Defektzone an der Entnahmestelle, sowie die fehlende Übereinstimmung von Knorpelschichtdicke und Ausrichtung der Kollagenfasern im Bereich der Entnahme- und Empfängerregion (Bös und Ellermann 2003). Hier besteht die Limitierung in der potentiellen Desintegration des Transplantates aufgrund des Zelltodes der Chondrozyten an der Grenzschicht (Huntley et al. 2005). Der entscheidende limitierende Faktor der Knorpel-Knochen-Transplantation ist die begrenzte Anzahl der zur Verfügung stehenden Zylinder. Vorteilhaft ist jedoch das einzeitige Vorgehen mit den entsprechend niedrigeren Kosten, das geringere Risiko einer Infektion oder Krankheitsübertragung und die sofortige Wiederherstellung der ursprünglichen hyalinen Gelenkkontur (Rolauffs et al. 2009). Weitere Vorteile der Knorpel-Knochen-Transplantation sind reproduzierbare und vergleichbar gute Ergebnisse in der Literatur (75-85\%), die rasche Einheilung und die daraus resultierende rasche Belastbarkeit, sowie die Eignung zur Therapie der Osteochondrosis dissecans bei tiefen, knöchernen Defekten (Bös und Ellermann 2003).

\subsubsection{Tissue Engineering}

Beim Tissue Engineering werden Prinzipien zur Entwicklung von biologischem Gewebeersatz angewandt. Natürliche Gewebe werden unter Laborbedingungen nachgezüchtet. Dazu dienen strukturelle Gerüste (Dreidimensionale Trägermaterialien) sowie lebende Zellen. Des Weiteren findet ein Eingriff in die Signaltransduktion statt. Hierbei ist insbesondere die autologe Chondrozytentransplantation zu erwähnen (Rolauffs et al. 2009). Die autologe Chondrozytentransplantation (ACl) wurde 1971 von Bentley und Greer publiziert (Aston und Bentley 1986). In der Anwendung unterscheidet man zwischen der freien und der matrix-gekoppelten Knorpelzelltransplantation (Hayes und Averett 2001). 


\subsubsection{Therapiealgorithmus}

Die differenzierte Therapieplanung schließt eine exakte Beschreibung des Knorpelschadens ein. Hier sollten Informationen hinsichtlich Defektausmaß, anatomischer Lokalisation, statischer Relevanz und zur Tiefenausdehnung vorliegen. Die Läsionsgröße stellt den entscheidenden Faktor für die Wahl des Operationsverfahrens dar. Kleine Defekte $\left(2-3 \mathrm{~cm}^{2}\right)$ sind insbesondere bei Kindern und Jugendlichen eine Indikation zur Mikrofrakturierung. Bei einer Defektgröße von $3-4 \mathrm{~cm}^{2}$ besteht die Indikation zur Knorpel-Knochen-Transplantation. Die Indikation zur autologen Chondrozytentransplantation besteht bei Läsionen bis zu einer Defektgröße von 15 $\mathrm{cm}^{2}$ (Albrecht und Weise 2008). Die spezielle Indikation der Knorpel-KnochenTransplantation stellen Knorpelschäden in den Belastungszonen der Femurkondylen (Ahmad et al. 2001) und der Patella im Stadium III und IV nach ICRS dar. Diese sollten eine Größe von $2 \times 2 \mathrm{~cm}$ nicht überschreiten. Bei Defekten bis zu $9 \mathrm{~cm}^{2}$ ist zudem eine Therapie mit der Mega-OATS-Technik möglich (Braun et al. 2007). Die Indikation zur Knorpel-Knochen-Transplantation besteht bei fokalen chondralen bzw. osteochondralen Defekten in den Belastungszonen des Kniegelenkes. Das Patientenalter sollte im Optimalfall nicht mehr als 50 Lebensjahre sowie das Defektausmaß nicht mehr als $1-4 \mathrm{~cm}^{2}$ betragen. Des Weiteren ist es obligat, Komorbiditäten wie Meniskus- und Bandverletzungen gleichzeitig zu sanieren. In Hinblick auf die Rehabilitationsphase ist eine entsprechende Patientenkompliance von gesonderter Bedeutung. Absolute Kontraindikationen stellen insbesondere Tumoren, Infektionen, Rheumatoide Arthritis, Arthrose, Defekte größer als $8 \mathrm{~cm}^{2}$ aber auch die Inkompliance des Patienten dar. Als relative Kontraindikation wird z. B. ein Patientenalter zwischen dem vierzigsten und fünfzigsten Lebensjahr angesehen. Des Weiteren werden $u$. a. eine Defektgröße zwischen 4 und $8 \mathrm{~cm}^{2}$ und das Vorliegen von gering ausgeprägten arthrotischen Veränderungen als relative Kontraindikationen diskutiert (Hangody et al. 2004). 


\subsubsection{Rehabilitation nach Knorpel-Knochen-Transplantation}

Postoperativ erfolgt zunächst die Hochlagerung und Kühlung der betroffenen Extremität. Am ersten postoperativen Tag kann mit isometrischen Spannungsübungen und dem Training auf der Motorbewegungsschiene (CPM) bei freiem passiven Bewegungsausmaß begonnen werden. Am zweiten postoperativen Tag sind die Wundkontrolle und die Entfernung der Drainagen obligat. Es schließt sich eine Mobilisation des Patienten an Unterarmgehstützen mit $15 \mathrm{kp}$ Teilbelastung an. Zusätzlich sollte eine adäquate Lymphdrainage durchgeführt werden. Ab dem zwölften postoperativen Tag ist nach Entfernung des Nahtmaterial die Steigerung der Belastung möglich (Bläsius et al. 2008). Bis zum Erreichen der Vollbelastung sollte zunächst jedoch eine Teilbelastung für 6 Wochen eingehalten werden. Zur Erhaltung und Verbesserung der Mobilität wird in der weiteren ambulanten Behandlung eine Motorbewegungsschiene als sinnvoll erachtet (Braun et al. 2007).

\subsubsection{Prognose nach Knorpel-Knochen-Transplantation}

Als wichtigster Faktor hinsichtlich des Therapieergebnisses gilt das optimale Einpassen der Zylinder in den Defekt. Gute und exzellente mittelfristige Ergebnisse gibt es zu 92 \% im Bereich der Femurkondylen, zu 88 \% im Tibia-Areal sowie zu 81 \% im Bereich der Patella bzw. der Trochlea (Böttner 2008). 


\section{HYPOTHESE}

Der therapeutische Langzeit-Effekt von bis zu 9,3 Jahren nach Knorpel-KnochenTransplantation wurde bisher in nur wenigen Studien beurteilt (Marcacci et al. 2007, Szerb et al. 2005, Hangody und Füles 2003, Meenen und Rischke 2003). Die Objektivierung des Therapieeffektes erfolgte anhand geläufiger Fragebögen (BrittbergScore, Tegner-Aktivitätsscore, Lysholm-Score, IKDC-Score, ICRS-Score).

Ziel der folgenden Untersuchung ist die Ermittlung des klinischen LangzeitTherapieeffektes der Knorpel-Knochen-Transplantation in der Behandlung der osteochondralen Läsion des Kniegelenkes (Grad III und Grad IV nach Outerbridge). Bei einem maximalen Nachuntersuchungszeitraum von 9,3 Jahren betrug das durchschnittliche Nachuntersuchungsintervall sieben Jahre. Als subjektive Messgröße wurde die Lebensqualität gewählt. Als objektive Messgrößen dienten der funktionelle Status des Gelenkes sowie die vorhandene Knorpelstruktur mit entsprechender Beurteilung der Zylinder-Lokalisation, der Zylinder-Integration und der Beurteilung des subchondralen Ödems. Als Messinstrument für die Untersuchungen wurde der ICRSEvaluierungs-Fragebogen benutzt. Des Weiteren wurde anhand dieser Arbeit geprüft, inwieweit die Variablen Defektgröße, Patientenalter zum Operationszeitpunkt, Body-Maß-Index sowie die Zeitspanne zwischen dem auslösenden Ereignis und der operativen Intervention den Therapieeffekt beeinflussen.

Es wird angenommen, dass die Therapie eines osteochondralen Defektes mit der Knorpel-Knochen-Transplantation nach durchschnittlich sieben Jahren mit einer signifikanten Verbesserung $(p<0,05)$ folgender Parameter einhergeht:

- Lebensqualität

- Gelenkfunktion

- Knorpelstruktur.

Des Weiteren wird postuliert, dass die Höhe des Patientenalters, die Höhe des BodyMass-Index, das Ausmaß der Defektgröße, sowie die Zeitspanne zwischen auslösendem Ereignis und der operativen Intervention langfristig einen signifikanten Einfluss $(p<0,05)$ auf das postoperative Ergebnis (Lebensqualität, Gelenkfunktion und Knorpelstruktur) ausüben. 


\section{MATERIAL UND METHODEN}

\subsection{EIN- UND AUSSCHLUSSKRITERIEN}

Einschlusskriterien:

- osteochondrale Läsion des Kniegelenkes im Stadium III und IV nach Outerbridge oder Osteochondrosis dissecans

- Operation in standardisiertem Verfahren (Mosaikplastik-System der Firma Smith \& Nephew)

- Operation im Zeitraum von 03/1998 bis 09/2001

- ein Operateur

- Nachuntersuchungszeitraum > 50 Monate

Ausschlusskriterien:

- unvollständige Dokumentation.

\subsection{AUSWERTUNGSSKALEN}

Zur Datenauswertung wurde der Analysebogen der International Cartilage Repair Society aus dem Jahr 2000 benutzt (siehe Anhang). Dieser gilt als zuverlässiges Messinstrument und eignet sich besonders, um Aussagen über individuelle Patienteneinschätzungen treffen zu können (Smith et. al 2005). Der Gesamtfragebogen besteht aus folgenden Teilen:

- IKDC (Fragebogen zur Funktion des Kniegelenkes)

- SF-36 (Fragebogen zum Gesundheitszustand)

- ICRS (Bewertung der Knorpelstruktur).

Der Gesamtbogen gliedert sich zudem in den Patienten- und Arztteil. Im ersten Teil werden Daten zum Verletzungshergang bzw. zum ersten Auftreten der Symptome, sowie Daten zur subjektiven Gesundheitseinschätzung erfasst. Im zweiten Teil werden anamnestische Daten erfasst, klinische und radiologische Untersuchungsbefunde erhoben, Daten zur Lokalisation des Schadens erfasst und die Schädigung klassifiziert. 


\subsubsection{SF-36}

Der SF-36-Fragebogen zum Gesundheitszustand basiert auf den Lebensqualitätsforschungen der vergangen Jahrzehnte. Dieser wurde 1998 von Bullinger und Kirchberger ins Deutsche übersetzt, für die deutsche Bevölkerung normiert und ist seitdem ein wissenschaftlich fundiertes, standardisiertes und valides Messinstrument. „Der SF-36 Gesundheitsfragebogen gehört zu den krankheitsübergreifenden Verfahren, die die subjektive Gesundheit verschiedener Populationen unabhängig von ihrem Gesundheitszustand aus der Sicht der Betroffenen erfassen. Insofern ist der Einsatzbereich auf die Population breit; sowohl gesunde Personen im Alter von 14 bis zum höchsten Lebensalter können mit dem Verfahren untersucht werden, als auch erkrankte Populationen unterschiedlicher Erkrankungsgruppen." (Bullinger und Kirchberger 1998, S. 10) Er kann bei ambulanten und stationären Patienten benutzt werden. Zur Datenerfassung gibt es mehrere Möglichkeiten (Fragebogen, Interview, Fremdbericht). Der Fragebogen besteht aus insgesamt 36 Fragen zu je acht verschiedenen Subskalen (Tab. 6) und einer Einzelbewertung (Bullinger und Kirchberger 1998).

\begin{tabular}{|ll|}
\hline Körperliche Funktionsfähigkeit & $(10)$ \\
Körperliche Rollenfunktion & $(4)$ \\
Körperliche Schmerzen & $(2)$ \\
Allgemeine Gesundheitswahrnehmung & $(5)$ \\
Vitalität & $(4)$ \\
Soziale Funktionsfähigkeit & $(2)$ \\
Emotionale Rollenfunktion & $(3)$ \\
Psychisches Wohlbefinden & $(5)$ \\
Veränderung der Gesundheit & $(1)$ \\
\hline
\end{tabular}

Tab. 6: $\quad$ Subskalen des SF-36

(mit entsprechendem Fragenanteil)

Die Datenauswertung ist mit Hilfe der Syntax für SPSS möglich. Tabelle 7 (S. 30) stellt die Kodierung der einzelnen Frage in der SPSS-Syntax dar. Bei der Dateneingabe entspricht It. Handanweisung jede angekreuzte Antwort einem vorkodierten Wert. Ein höherer Wert korreliert mit einem besseren Gesundheitszustand. Des Wei- 
teren müssen sieben Items umkodiert werden, weil diese entgegengesetzt gewertet sind.

Zur End-Kontrolle sollten alle Werte manuell berechnet und anschließend abgeglichen werden. Dies erfolgt It. Bullinger und Kirchberger (Bullinger und Kirchberger 1998) in 3 nachfolgenden Schritten:

- Umkodierung und Rekalibrierung von zehn Fragen

- Berechnung von Skalenwerten durch Addition der Skalenrohwerte

- Umrechnung der Skalenrohwerte in transformierte Skalenwerte.

Die Transformation der Skalenwerte wird nach dem in der Handanweisung angegebenen Algorithmus durchgeführt (Abb. 13). Ein höherer Wert entspricht einem besseren Gesundheitszustand.

\begin{tabular}{|c|c|c|c|c|c|c|}
\hline Eigene Kontrollkodierung & SPSS-Kürzel & Name & ItemzahI & Fragenummer & SPSS-Kürzel & Fragenummer \\
\hline $\mathrm{A}$ & KÖFU & $\begin{array}{l}\text { functional functioning } \\
\text { (Körperl. Funktionsfähigkeit) }\end{array}$ & 10 & $3,4,5,6,7,8,9,10,11,12$ & pfi01-pfi 10 & $3 a-3 j$ \\
\hline c & EMRO & $\begin{array}{l}\text { role functioning emotional } \\
\text { (Emotionale Rollenfunktion) }\end{array}$ & 3 & $17,18,19$ & rolem 1-rolem3 & $5 a-5 c$ \\
\hline E & PSYC & $\begin{array}{l}\text { mental health } \\
\text { (Psychisches Wohlbefinden) }\end{array}$ & 5 & $24,25,26,28,30$ & mhi 1-mhi5 & $9 b, 9 c, 9 d, 9 f, 9 h$ \\
\hline $\mathrm{F}$ & SOFU & $\begin{array}{l}\text { social functioning } \\
\text { (Soziale Rollenfunktion) }\end{array}$ & 2 & 20,32 & $\operatorname{soc} 1, \operatorname{soc} 2$ & 6,1 \\
\hline $\mathrm{H}$ & AGES & $\begin{array}{l}\text { general health } \\
\text { (Allgemeine Gesundheit) }\end{array}$ & 5 & $1,33,34,34,35,36$ & ghp1-ghp5 & $1,11 \mathrm{a}-11 \mathrm{~d}$ \\
\hline & & $\begin{array}{l}\text { reorted health transition } \\
\text { (veränderung } \mathrm{d} \text {. Gesundh.) }\end{array}$ & 1 & 2 & hchange1 & 2 \\
\hline
\end{tabular}

Tab. 7: $\quad$ Kodierung der Fragen zur Bearbeitung mit SPSS

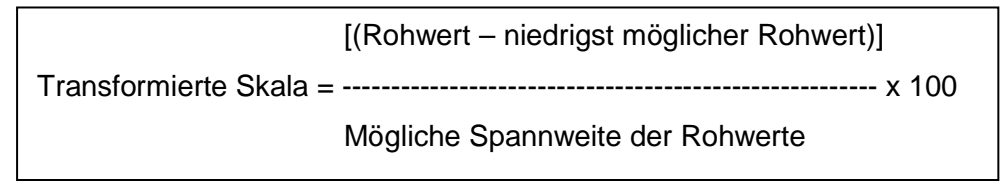

Abb. 13: Transformationsalgorithmus der Skalenwerte des SF- 36 


\subsubsection{IKDC}

Der IKDC-Fragebogen umfasst die subjektive Funktionsbeurteilung des Kniegelenkes. Es werden insbesondere Aussagen über die Mobilität und Belastungsfähigkeit des Kniegelenkes erfasst (Tab. 8).

\begin{tabular}{|c|l|}
\hline 1 & höchste Aktivitätsstufe, die ohne erhebliche Schmerzen im Knie ausgeübt werden kann \\
\hline 2 & Häufigkeit der Schmerzen in den vergangenen 4 Wochen oder seit dem Auftreten der Verletzung \\
\hline 3 & Stärke der Schmerzen \\
\hline 4 & Gelenksteife oder Schwellung des Kniegelenkes in den vergangenen 4 Wochen oder seit dem Auftreten der Verletzung \\
\hline 5 & höchste Aktivitätsstufe, die ohne erhebliches Anschwellen des Knies ausüben werden kann \\
\hline 6 & Auftreten von Blockaden oder Giving-Way-Phänomenen \\
\hline 7 & höchste Aktivitätsstufe, die ohne erhebliche durch Knieschwäche verursachte Gangunsicherheit eingehalten werden kann \\
\hline 8 & höchste Aktivitätsstufe, an der der Patient regelmäßig teilnehmen kann \\
& $\begin{array}{l}\text { Beurteilung der Schwierigkeit von: Treppensteigen, Treppe hinuntergehen, auf dem vorderen Knie knien, Hockstellung, } \\
\text { schnell anhalten und starten }\end{array}$ \\
\hline 10 & Beurteilung der Funktionsfähigkeit des Knies aktuell und vor der Verletzung \\
\hline
\end{tabular}

Tab. 8: $\quad$ Fragenkategorien zur subjektiven Funktionsbeurteilung

Die Berechnungen der Ergebnisse für dieses Formblatt erfolgen anhand der im Fragebogen dargestellten Anleitung. Jede Antwort der einzelnen Fragen erhält einen Zahlenwert, wobei eins die niedrigste Funktionsstufe oder höchste Symptomstufe darstellt. Zur Ermittlung des Gesamtergebnisses addiert man die Einzelpunkte (Rohergebnisse) und transformiert die Werte anschließend in eine Skala von null bis einhundert (Abb. 14). Die Antworten in Frage 10 fließen nicht in die Gesamtbeurteilung ein.

IKDC-Ergebnis $=\frac{\text { Rohergebnis }- \text { Niedrigstmögliche Punktzahl }}{\text { Punktzahlbereich }} \times 100$

Abb. 14: Transformationslogarithmus der Skalenwerte des IKDC-Rohwertes 
Nach der Umwandlung entspricht das Ergebnis der Funktionsfähigkeit des Kniegelenkes. Höhere Punktzahlen beschreiben eine höhere Funktionsfähigkeit und somit geringere Symptome.

Das Gesamtbewertungspaket (siehe Anhang) umfasst des Weiteren ein Arbeitsblatt zur Erfassung der in der klinischen Untersuchung erhobenen Daten (IKDC-Wert der klinischen Untersuchung). Dadurch werden die Daten mit späteren Untersuchungen vergleichbar gemacht. Die klinische Untersuchung umfasst:

- Schwellung

- Bewegungsausmaß

- Banduntersuchung

- Kompartmentuntersuchung

- Pathologische Veränderung im Entnahmebereich

- Beurteilung der Röntgenaufnahmen

- Funktioneller Test .

Es wird zwischen vier Gruppengraden differenziert. Grad A und Grad B entsprechen normaler bzw. annähernd normaler Kniefunktion. Die Grade C und D sind mit abnormaler bzw. stark abnormaler Kniefunktion korreliert. Zuerst erfolgt die Bestimmung des Gruppengrades. Dieser ist durch den geringsten Grad in der Gruppe definiert. In die Endbewertung gehen nur die Gruppengrade der Punkte eins bis drei ein. Der schlechteste Gruppengrad bestimmt den Endgrad. 


\subsubsection{ICRS}

Dieser Beurteilungsbogen (siehe Anlage) erlaubt die Protokollierung und Auswertung unterschiedlicher Knorpelersatz- bzw. Knorpelreparaturverfahren. Dieser gliedert sich deshalb in Protokoll A und B. In der Studie wurde Protokoll B (Beurteilung des Knorpelzustandes bei stattgehabter Mosaikplastik) benutzt. Die Bewertung ist in vier Bereiche eingeteilt, in denen jeweils null bis vier Punkte vergeben werden können:

- Tiefe des verbliebenen Defektes im Vergleich zur Umgebung

- Vitalität des Transplantates

- Gewebsintegration

- Beschaffenheit der Oberfläche.

Maximal können 12 Punkte erreicht werden. Folglich kann zwischen normaler (12 Punkte), fast normaler (11 bis 8 Punkte), abnormaler ( 7 bis 4 Punkte) und stark abnormaler (3 bis 1 Punkte) Knorpelbeschaffenheit differenziert werden. Der ICRSScore wird heute als Standardinstrument in der Beurteilung der Knorpelstruktur angesehen und ist ein statistisch sicherer Test mit entsprechender Reliabilität und reproduzierbaren Ergebnissen (Smith et al. 2005). 


\subsection{PATIENTENKOLLEKTIV}

Die Studie umfasst ein Patientenkollektiv von 38 Patienten, wovon aufgrund unvollständiger Daten 31 Patienten (21 Männer und 10 Frauen) nachuntersucht werden konnten. Sämtliche operative Eingriffe wurden durch einen Operateur im Zeitraum von März 1998 bis September 2001 in der Orthopädischen Klinik der Universitätsmedizin Göttingen durchgeführt. Zur operativen Therapie wurde die Knochen-KnorpelTransplantation angewandt. Bei allen Patienten lagen Knorpelschäden im Stadium III $(n=11)$ oder IV $(n=20)$ nach Outerbridge vor. Diese wurden in $n=26$ Fällen als umschriebene osteochondrale Läsion und in $n=5$ Fällen als Osteochondrosis dissecans klassifiziert. Bei allen Patienten bestanden monolokale Schädigungen. Das initiale Schmerzereignis bzw. Trauma trat in $n=18$ fällen in der Freizeit, in $n=11$ Fällen beim Sport und in $n=2$ Fällen während der Arbeit auf (Abb. 15).

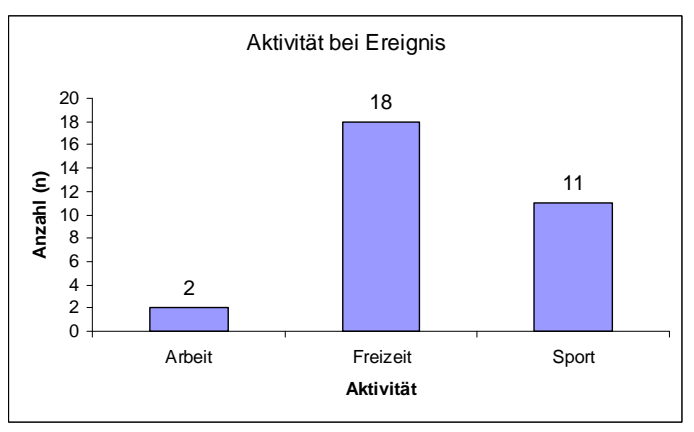

Abb. 15: Aktivität der Patienten zum Zeitpunkt der Verletzung

Im Mittel lagen 53,26 Tage zwischen Unfallereignis bzw. dem Auftreten erster Symptome und dem Operationszeitpunkt. Die meisten der Patienten (nahezu $50 \%$ ) wurden zwischen dem dritten und sechsten Monat nach der klinischen Beschwerdemanifestation der operativen Versorgung zugeführt (Abb. 16).

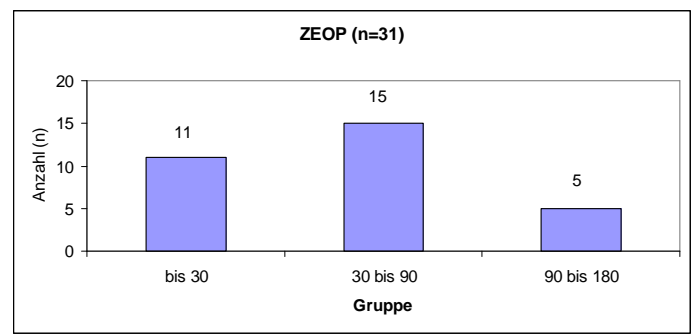

Abb. 16: Zeitraum zwischen Schmerzereignis bzw.

Trauma und OP in Tagen 
Das Durchschnittsalter der Patienten lag zum Operationszeitpunkt bei 35 Lebensjahren. $\mathrm{N}=11$ Patienten befanden sich zum Operationszeitpunkt in der Altersgruppe bis zu 29 Lebensjahren. In der Gruppe der 30- bis 39jährigen war n=8 und in der Gruppe der 40 bis 49 jährigen waren $n=9$ Patienten vertreten. Älter als 50 Lebensjahre waren $\mathrm{n}=3$ Patienten (Abb. 17). Das maximale Patientenalter zum Operationszeitpunkt lag bei 53 Lebensjahren.

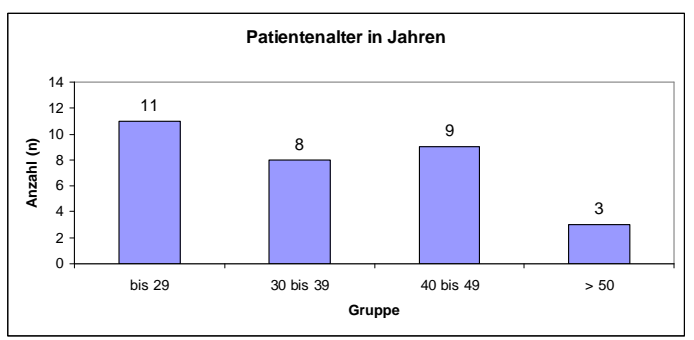

Abb. 17: Patientenanzahl nach Altersgruppen zum Operationszeitpunkt $(n=31)$

Der Durchschnittswert des Body-Mass-Index der Patienten belief sich zum OPZeitpunkt auf $24,89 \mathrm{~kg} / \mathrm{m}^{2}$, wobei bei $\mathrm{n}=18$ Patienten ein Normalgewicht (BMI 18,5$24,99 \mathrm{~kg} / \mathrm{m}^{2}$ ) und bei $\mathrm{n}=13$ Patienten ein Übergewicht im Sinne von Prä-Adipositas $\left(\mathrm{BMl} \geq 25-29,99 \mathrm{~kg} / \mathrm{m}^{2}\right)$ bzw. Adipositas (BMl $\geq 30 \mathrm{~kg} / \mathrm{m}^{2}$ ) vorlag (Abb. 18).

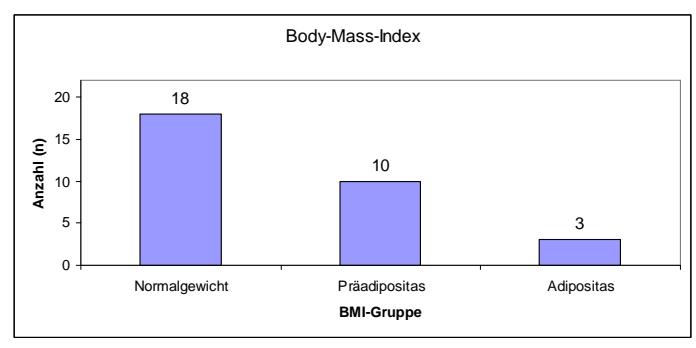

Abb. 18: Patientenanzahl nach Body-Mass-Index zum Operationszeitpunkt $(n=31)$

Die in Kapitel 1.2.1 angeführten epidemiologischen Eckdaten finden sich in dieser Arbeit nahezu identisch wieder (vgl. Tab. 2, Seite 11). Die Knorpelverletzungen waren vorrangig $(n=18)$ im Bereich der Hauptbelastungszone des medialen Femurkondylus (MFC), in $n=8$ Fällen im Bereich der Patella sowie in $n=5$ Fällen im Bereich der Hauptbelastungszone des lateralen Femurkondylus (LFC) lokalisiert (Abb. 19, S. 36). 


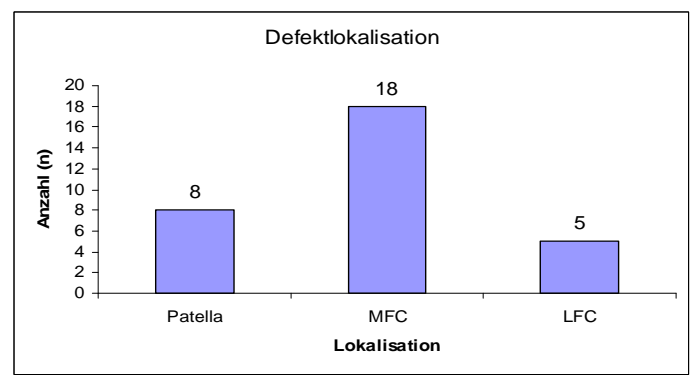

Abb. 19: Lokalisationen der osteochondralen Läsion $(n=31)$

Die durchschnittliche Defektgröße nach durchgeführtem Debridement betrug $3 \mathrm{~cm}^{2}$. In $\mathrm{n}=5$ Fällen wurden Defekte bis zu $2 \mathrm{~cm}^{2}$ behandelt. $\mathrm{N}=16$ Läsionen wurden im Bereich zwischen 2 und $4 \mathrm{~cm}^{2}$ sowie $\mathrm{n}=10$ Fälle im Bereich zwischen 4 und $6 \mathrm{~cm}^{2}$ eingruppiert. Die maximale Defektgröße einer Läsion betrug $4,8 \mathrm{~cm}^{2}$ (Abb. 20).

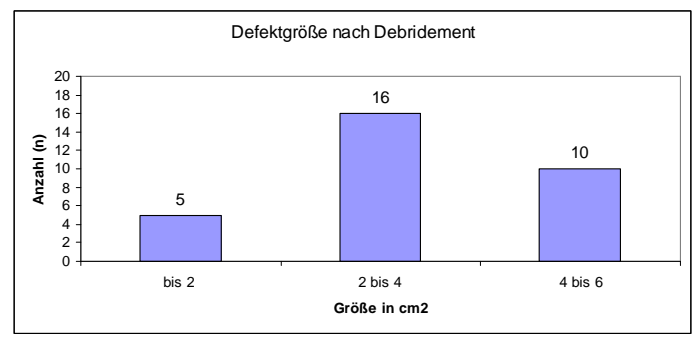

Abb. 20: Größe nach Debridement $(n=31)$

In 17 Fällen waren zusätzliche operative Maßnahmen wie beispielsweise Knorpelund Meniskusglättungen, Meniskusteilresektionen oder achskorrigierende Maßnahmen indiziert (Tab. 9).

\begin{tabular}{|l|l|}
\hline MP & $n=14$ \\
MP, Knorpelglättung & $n=3$ \\
MP, Knorpelglättung, Synovektomie & $n=2$ \\
MP, Knorpelglättung, Notch-Plastik & $n=1$ \\
MP, Meniskusglättung & $n=1$ \\
MP, Außenmeniskusteilresektion & $n=1$ \\
MP, Innenmeniskusteilresektion & $n=2$ \\
MP, Laterales release & $n=3$ \\
MP, Laterales release, mediale Kapselspaltung & $n=1$ \\
MP, VKB-Plastik & $n=1$ \\
MP, VKB-Plastik, Innenmeniskusteilresektion & $n=1$ \\
MP, TKO & $n=1$ \\
\hline
\end{tabular}

Tab. 9: $\quad$ Eingriffe und zusätzliche operative Maßnahmen (MP - Mosaikplastik, TKO - Tibiakopfosteotomie, VKB - Vorderes Kreuzband) 
Der Nachuntersuchungszeitraum erstreckte sich bis einschließlich Juni 2007, wobei der Zeitpunkt der letzten Nachuntersuchung im Durchschnitt bei sieben Jahren lag (Abb. 21).

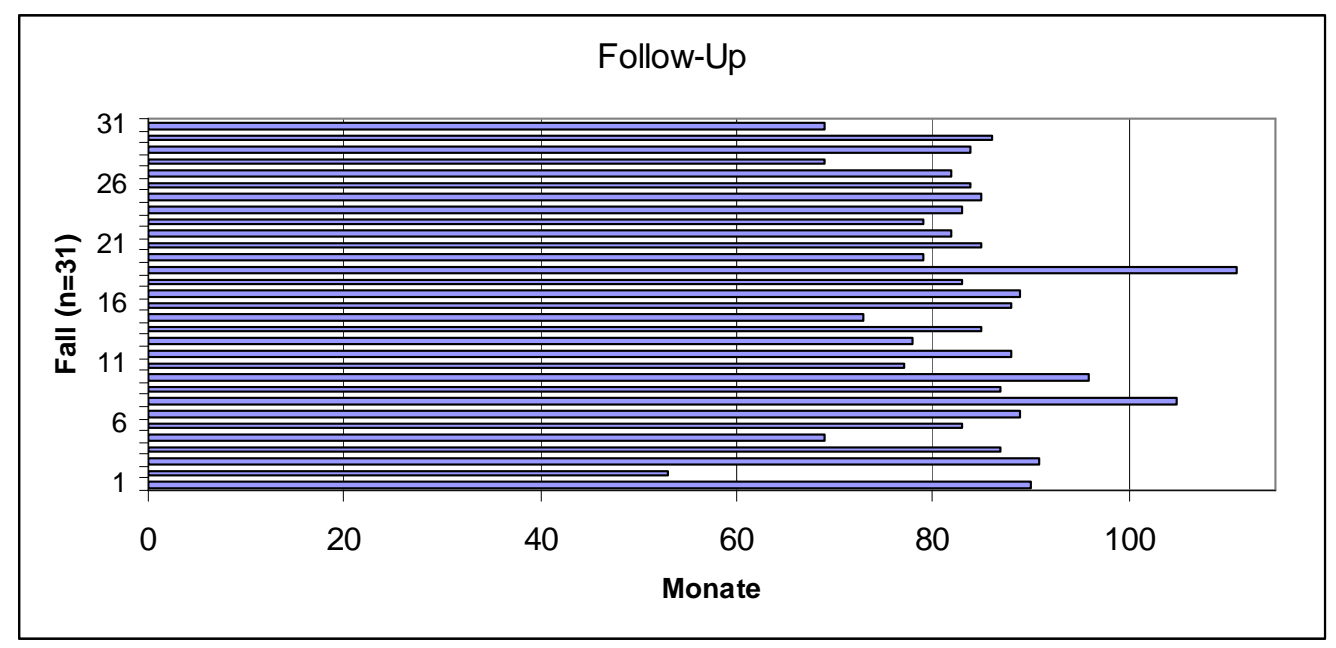

Abb. 21: Zeitraum der Nachuntersuchung (in Monaten)

Tabelle 10 und 11 geben eine Übersicht der über das Patientenkollektiv erhobenen Daten.

\begin{tabular}{|c|c|c|c|c|c|}
\hline & $\frac{\mathrm{POZ}}{\text { (in Jahren) }}$ & $\frac{\underline{\text { ZEOP }}}{\text { (in Tagen) }}$ & $\begin{array}{c}\underline{\text { FUP }} \\
\text { (in Monaten) }\end{array}$ & $\underset{\left(\text { in } \mathrm{cm}^{2}\right)}{\mathbf{D G}}$ & $\frac{\text { BMI }}{\left(\text { in } \mathrm{kg} / \mathrm{m}^{2}\right)}$ \\
\hline Mittelwert & 36,06 & 53,26 & 83,5 & 3 & 24,89 \\
\hline Median & 37 & 49 & 84 & 2,5 & 24,8 \\
\hline Minimum & 17 & 7 & 53 & 1 & 18,9 \\
\hline Maximum & 53 & 140 & 111 & 4,8 & 31,8 \\
\hline Spannweite & 36 & 133 & 58 & 3,8 & 12,9 \\
\hline
\end{tabular}

Tab. 10: Patientendaten (POZ - Patientenalter zum OP-Zeitpunkt, ZEOP - Zeit zwischen Ereignis und Operation, FUP - Nachuntersuchungszeitraum, DG - Defektgröße, BMI - Body-Mass-Index) 


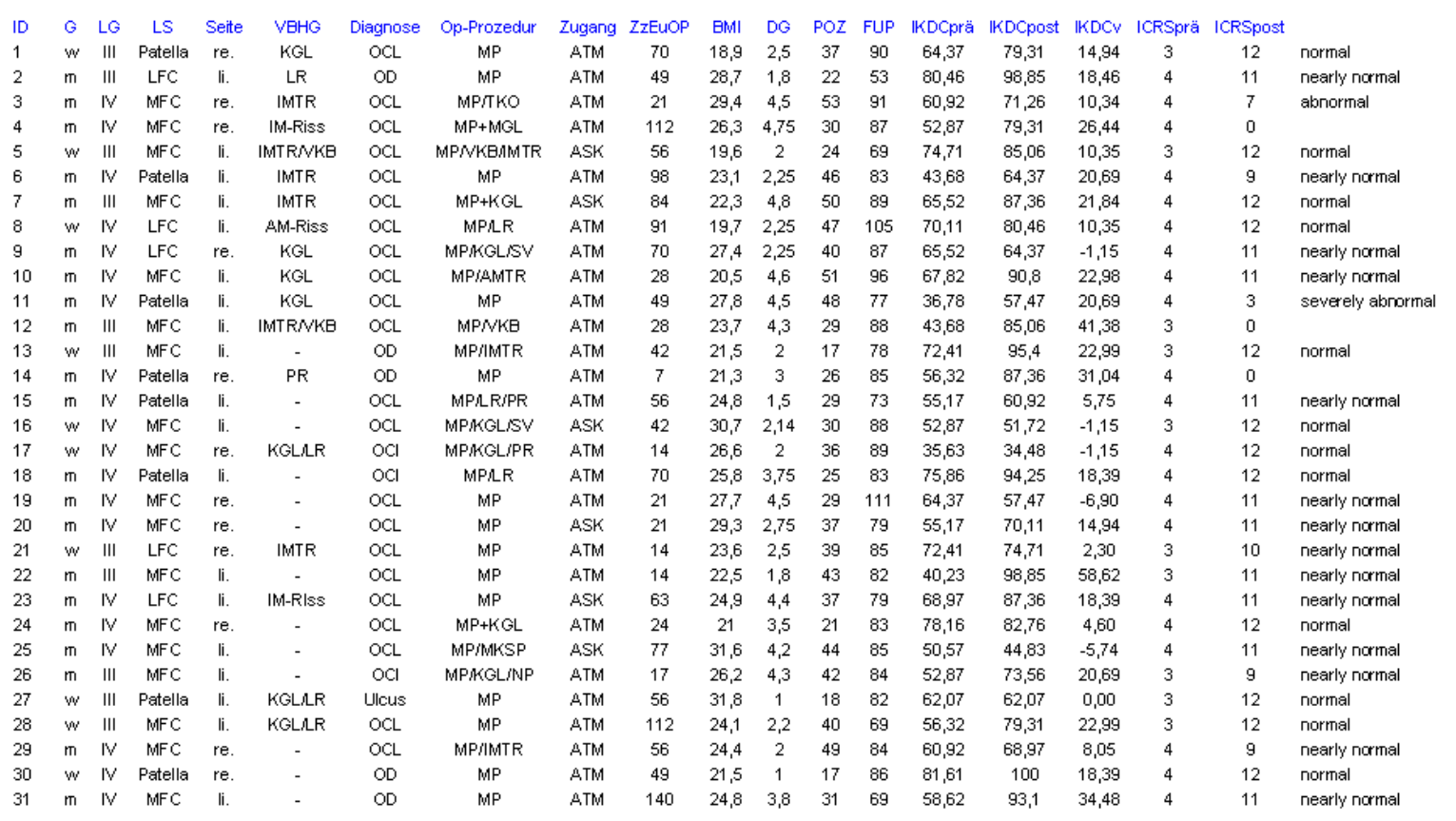

Tab. 11: Gesamtdatenlage des Patientenkollektivs

ID - Identifikationsnummer,

$\mathrm{G}$ - Geschlecht,

$L G$ - Läsionsgrad,

LS - Lokalisation,

VBHG - Vorbehandlung,

ZZeuOP - Zeit zwischen Ereignis und Operation (Monate)

$\mathrm{BMI}$ - Body-Mass-Index $\left(\mathrm{kg} / \mathrm{m}^{2}\right)$,

DG - Defektgröße $\left(\mathrm{cm}^{2}\right)$,

POZ - Patientenalter zum OP-Zeitpunkt (Jahre),

FUP - Follow-Up (Monate),

LFC - lateraler Femurkondylus,

MFC - medialer Femurkondylus,

OCL - osteochondrale Läsion,

OD - Osteochondrosis dissecans,

MP - Mosaikplastik,

TKO - Tibiakopfosteotomie,

MGL - Meniskusglättung,

KGL - Knorpelglättung,

LR - Laterales Release,

IMTR - Innenmeniskusteilresektion,

VKB - Plastik des vorderen Kreuzbandes,

SV - Synovektomie,

AMTR - Außenmeniskusteilresektion,

ATM - Arthrotomie,

ASK - Arthroskopie 


\subsection{STATISTIK}

Die Auswertung des SF-36-Fragebogens erfolgte entsprechend der Handanweisung zum SF-36 und der beiliegenden SPSS-Syntax. Die Werte des IKDC sowie des ICRS wurden mit Hilfe der beiliegenden Berechnungsvorschriften des Fragebogens berechnet bzw. erhoben.

Die Datenauswertung wurde mit Hilfe des Statistikprogramms SPSS (Version 14.0, SPSS Inc. Chicago IL, USA). Zur deskriptiven Darstellung und Analyse der Daten wurde Microsoft Excel 2003 verwandt. Die Resultate wurden mittels ANOVA (Analysis of variance) analysiert. Als statistische Tests dienten der t-Test, der WilcoxonTest für Paardifferenzen, die Korrelation nach Pearson sowie die Rangkorrelation nach Spearman. Das Signifikanzniveau wurde auf $p<0,05$ festgelegt. 


\section{ERGEBNISSE}

\subsection{ERGEBNISSE DES SF-36-FRAGEBOGENS}

Die Tabelle 12 stellt die prä- und postoperativ erreichten Punktwerte der acht Subund der beiden Summenskalen des SF-36 und die Werte im Bereich der Gesundheitsveränderung dar. Postoperativ ist als der Zeitpunkt der letzen Nachuntersuchung definiert (durchschnittlich sieben Jahre, maximal 9,3 Jahre). Ein höherer Skalenwert entspricht einer besseren Funktionsfähigkeit.

\begin{tabular}{|c|c|c|c|c|c|c|c|c|}
\hline & \multirow[b]{2}{*}{$\mathrm{N}$} & \multirow[b]{2}{*}{ Mittelwert } & \multirow{2}{*}{$\begin{array}{l}\text { Standardabw } \\
\text { eichung }\end{array}$} & \multirow[b]{2}{*}{ Minimum } & \multirow[b]{2}{*}{ Maximum } & \multicolumn{3}{|c|}{ Perzentile } \\
\hline & & & & & & 25. & 50. (Median) & 75. \\
\hline KÖFU prä & 31 & 81,45 & 20,744 & 10 & 100 & 70,00 & 90,00 & 95,00 \\
\hline KÖRO prä & 31 & 82,26 & 35,469 & 0 & 100 & 100,00 & 100,00 & 100,00 \\
\hline KÖSC prä & 31 & 64,23 & 14,092 & 22 & 84 & 51,00 & 62,00 & 72,00 \\
\hline AGES prä & 31 & 47,35 & 11,283 & 20 & 72 & 37,00 & 47,00 & 57,00 \\
\hline VITA prä & 31 & 76,29 & 6,187 & 60 & 85 & 70,00 & 75,00 & 80,00 \\
\hline SOFU prä & 31 & 85,484 & 12,1178 & 50,0 & 100,0 & 75,000 & 87,500 & 87,500 \\
\hline EMRO prä & 31 & 100,000 &, 0000 & 100,0 & 100,0 & 100,000 & 100,000 & 100,000 \\
\hline PSYC prä & 31 & 78,58 & 3,354 & 72 & 84 & 76,00 & 80,00 & 80,00 \\
\hline $\begin{array}{l}\text { Gesundheits- } \\
\text { veränderung prä }\end{array}$ & 31 & 4,61 & 495 & 4 & 5 & 4,00 & 5,00 & 5,00 \\
\hline $\begin{array}{l}\text { Körperliche } \\
\text { Summenskala prä }\end{array}$ & 31 & 43,6049 & 9,40247 & 13,45 & 53,26 & 42,7852 & 46,6306 & 48,8102 \\
\hline $\begin{array}{l}\text { Psychische } \\
\text { Summenskala prä }\end{array}$ & 31 & 56,3404 & 2,28854 & 51,75 & 62,56 & 54,8561 & 56,0076 & 57,4625 \\
\hline KÖFU post & 31 & 84,68 & 19,233 & 35 & 100 & 70,00 & 95,00 & 100,00 \\
\hline KÖRO post & 31 & 87,10 & 24,041 & 25 & 100 & 75,00 & 100,00 & 100,00 \\
\hline KÖSC post & 31 & 79,10 & 17,007 & 41 & 100 & 74,00 & 84,00 & 100,00 \\
\hline AGES post & 31 & 74,68 & 14,418 & 47 & 100 & 67,00 & 72,00 & 87,00 \\
\hline VITA post & 31 & 80,65 & 8,826 & 60 & 100 & 75,00 & 80,00 & 85,00 \\
\hline SOFU post & 31 & 88,710 & 14,1990 & 50,0 & 100,0 & 87,500 & 87,500 & 100,000 \\
\hline EMRO post & 31 & 94,624 & 15,1456 & 33,3 & 100,0 & 100,000 & 100,000 & 100,000 \\
\hline PSYC post & 31 & 79,61 & 7,544 & 52 & 92 & 80,00 & 80,00 & 84,00 \\
\hline $\begin{array}{l}\text { Gesundheits- } \\
\text { veränderung post }\end{array}$ & 31 & 2,03 & 948 & 1 & 4 & 1,00 & 2,00 & 3,00 \\
\hline $\begin{array}{l}\text { Körperliche } \\
\text { Summenskala post }\end{array}$ & 31 & 50,3252 & 8,17392 & 32,02 & 59,71 & 45,2189 & 53,8050 & 55,8111 \\
\hline $\begin{array}{l}\text { Psychische } \\
\text { Summenskala post }\end{array}$ & 31 & 55,4631 & 3,15439 & 47,62 & 60,63 & 53,9284 & 55,7513 & 57,2939 \\
\hline
\end{tabular}

Tab. 12: prä- und postoperative Daten der Sub- und Summenskalen des SF-36 (KÖFU - Körperliche Funktionsfähigkeit, KÖRO -Körperliche Rollenfunktion, EMRO - Emotionale Rollenfunktion, VITA - Vitalität, PSYC - Psychisches Wohlbefinden, SOFU - Soziale Rollenfunktion, KÖSC - Körperliche Schmerzen, AGES - Allgemeine Gesundheit) 
Im Vergleich der prä- und postoperativen Subskalenwerte „Körperliche Schmerzen“, „Allgemeine Gesundheitswahrnehmung“, „Vitalität“ und der „Körperlichen Summenskala" stellten sich signifikante Veränderungen dar (Abb. 22 und Abb. 23).

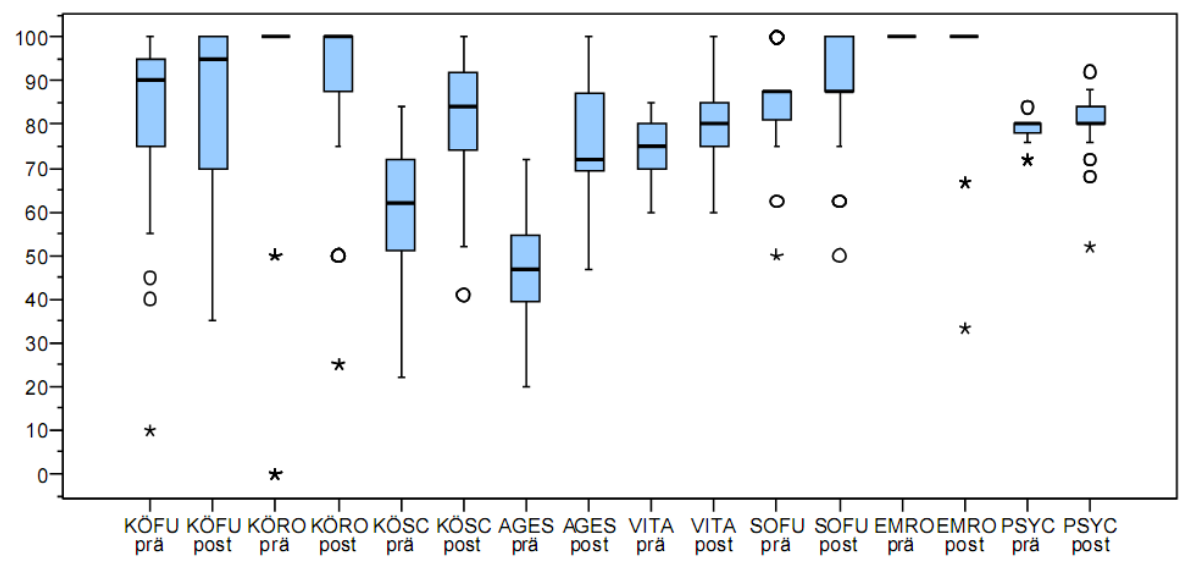

Abb. 22: $\quad$ prä- und postoperative Werte der

Subskalen des SF-36

signifikanter Unterschied in KÖSC $(p<0,001)$,

AGES $(p<0,001)$ und VITA $(p=0,008)$;

$\left({ }^{*}\right.$ - extremer Wert, o - Ausreißer)

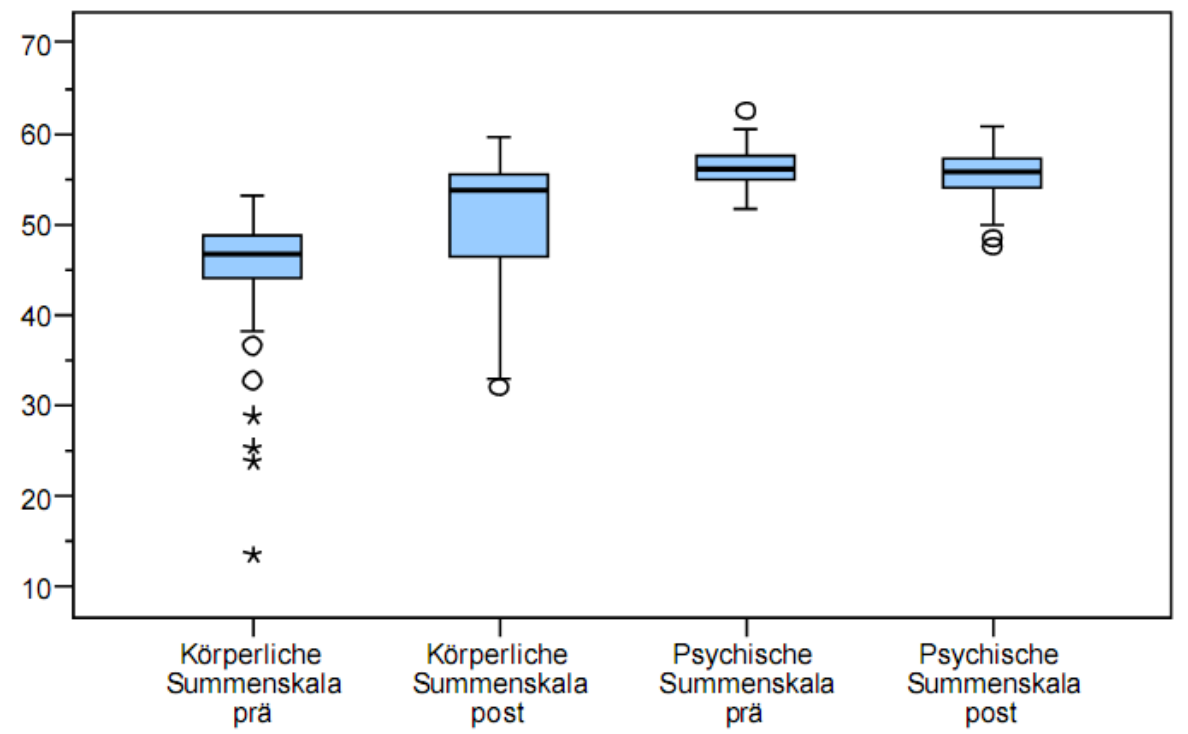

Abb. 23: $\quad$ prä- und postoperative Werte der

Summenskalen des SF-36

signifikanter Unterschied in der

Körperlichen Summenskala $(p<0,001)$;

( ${ }^{*}$ - extremer Wert, o - Ausreißer) 
In den meisten Subskalen sowie in der „Körperlichen Summenskala“ stellten sich signifikante Abweichungen von einer Normalverteilung heraus, so dass die Messungen zu beiden Zeitpunkten nicht parametrisch mittels Wilcoxon-Test für Paardifferenzen verglichen wurden. Dabei zeigten sich signifikant positive Unterschiede zwischen den prä- und postoperativen Subskalenwerten „Körperliche Schmerzen“ ( $p<0,001$, Wilcoxon-Test), „Allgemeine Gesundheit“ ( $p<0,001$, WilcoxonTest) und „Vitalität“ ( $p=0,008$, Wilcoxon-Test).

Des Weiteren konnten signifikante Unterschiede der Skalenwerte in der „Körperlichen Summenskala“ nachgewiesen werden ( $p<0,001$, Wilcoxon-Test, (Abb. 23)). In den übrigen Subskalen („Körperliche Funktionsfähigkeit“, „Körperliche Rollenfunktion“, „Soziale Funktionsfähigkeit“ und „Emotionale Rollenfunktion“) sowie der „Psychischen Summenskala“ zeigten sich keine signifikanten Unterschiede.

Abbildung 24 und 25 (S. 43) stellen den Vergleich zwischen dem postoperativen Punktwert der SF-36-Subskalen und den Werten der deutschen Normbevölkerung dar. Im Vergleich der postoperativen Punktwerte des SF-36 des Studienkollektivs und der deutschen Normbevölkerung (Tab. 13) bestehen signifikante Unterschiede in den Subskalen „Allgemeine Gesundheitswahrnehmung“ ( $p=0,046$, U-Test), „Vitalität“ $(p<0,001, U$-Test) und „Psychisches Wohlbefinden“ ( $p=0,040, U$-Test) sowie in den „Psychischen Summenskalen“ ( $p<0,001$, U-Test, Abb. 26).

\begin{tabular}{|c|c|c|c|c|c|c|c|c|c|}
\hline \multirow[b]{2}{*}{ Gruppe } & & \multirow[b]{2}{*}{$\mathrm{N}$} & \multirow[b]{2}{*}{ Mittelwert } & \multirow{2}{*}{$\begin{array}{l}\text { Standardabw } \\
\text { eichung }\end{array}$} & \multirow[b]{2}{*}{ Minimum } & \multirow[b]{2}{*}{ Maximum } & \multicolumn{3}{|c|}{ Perzentile } \\
\hline & & & & & & & 25. & 50. (Median) & 75. \\
\hline \multirow[t]{11}{*}{ Patienten } & KOFU post & 31 & 84,68 & 19,23 & 35 & 100 & 70,00 & 95,00 & 100,00 \\
\hline & KORO post & 31 & 87,10 & 24,04 & 25 & 100 & 75,00 & 100,00 & 100,00 \\
\hline & KOSC post & 31 & 79,10 & 17,01 & 41 & 100 & 74,00 & 84,00 & 100,00 \\
\hline & AGES post & 31 & 74,68 & 14,42 & 47 & 100 & 67,00 & 72,00 & 87,00 \\
\hline & VTA post & 31 & 80,65 & 8,83 & 60 & 100 & 75,00 & 80,00 & 85,00 \\
\hline & SOFU post & 31 & 88,71 & 14,20 & 50,0 & 100,0 & 87,50 & 87,50 & 100,00 \\
\hline & EMRO post & 31 & 94,62 & 15,15 & 33,3 & 100,0 & 100,00 & 100,00 & 100,00 \\
\hline & PSYC post & 31 & 79,61 & 7,54 & 52 & 92 & 80,00 & 80,00 & 84,00 \\
\hline & GV post & 31 & 2,03 & .95 & 1 & 4 & 1,00 & 2,00 & 3,00 \\
\hline & KSK post & 31 & 50,33 & 8,17 & 32,02 & 59,71 & 45,22 & 53,80 & 55,81 \\
\hline & PSK post & 31 & 55,46 & 3,15 & 47,62 & 60,63 & 53,93 & 55,75 & 57,29 \\
\hline \multirow{11}{*}{$\begin{array}{l}\text { Dt. Norm- } \\
\text { bevolkerung }\end{array}$} & KOFU post & 2908 & 83,58 & 23,87 & 0 & 100 & 75,00 & 95,00 & 100,00 \\
\hline & KORO post & 2900 & 80,56 & 34,53 & 0 & 100 & 75,00 & 100,00 & 100,00 \\
\hline & KOSC post & 2905 & 77,15 & 28,50 & 0 & 100 & 52,00 & 100,00 & 100,00 \\
\hline & AGES post & 2913 & 66,05 & 21,17 & 0 & 100 & 52,00 & 71,25 & 82,00 \\
\hline & VTA post & 2888 & 61,75 & 19,21 & 0 & 100 & 50,00 & 65,00 & 75,00 \\
\hline & SOFU post & 2911 & 87,67 & 19,45 & .0 & 100,0 & 87,50 & 100,00 & 100,00 \\
\hline & EMRO post & 2899 & 87,74 & 28,96 & .0 & 100,0 & 100,00 & 100,00 & 100,00 \\
\hline & PSYC post & 2900 & 72,80 & 17,38 & 0 & 100 & 64,00 & 76,00 & 84,00 \\
\hline & GV post & 2914 & 2,90 & 99 & 1 & 5 & 2,00 & 3,00 & 4,00 \\
\hline & KSK post & 2861 & 49,20 & 10,93 & 5,33 & 68,72 & 44,15 & 53,38 & 57,08 \\
\hline & PSK post & 2861 & 50,97 & 8,77 & 11,85 & 73,25 & 47,80 & 52,99 & 56,71 \\
\hline
\end{tabular}

Tab. 13: postoperative Sub- und Summenskalen im Vergleich mit der deutschen Normbevölkerung 


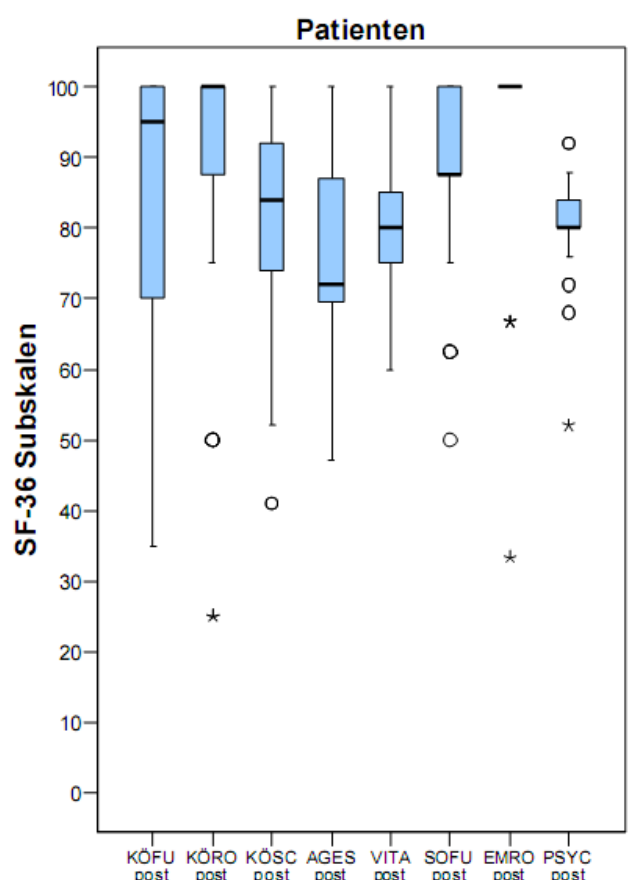

Dt. Normbevölkerung

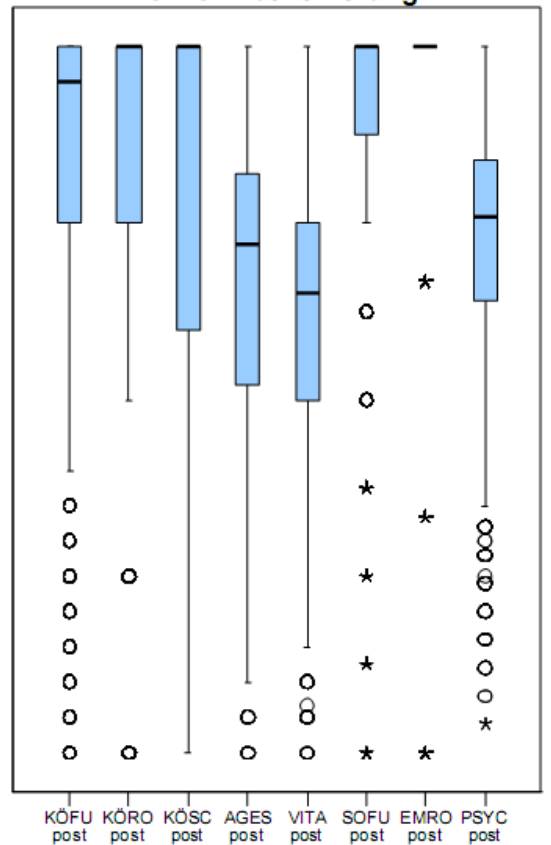

Abb. 24: postoperative Subskalenwerte im Vergleich mit der deutschen Normbevölkerung; signifikante Unterschiede in den Subskalen AGES $(p=0,046)$, Vitalität $(p<0,001)$ und PSYC $(p=0,040)$, $\left({ }^{*}\right.$ - extremer Wert, o - Ausreißer)
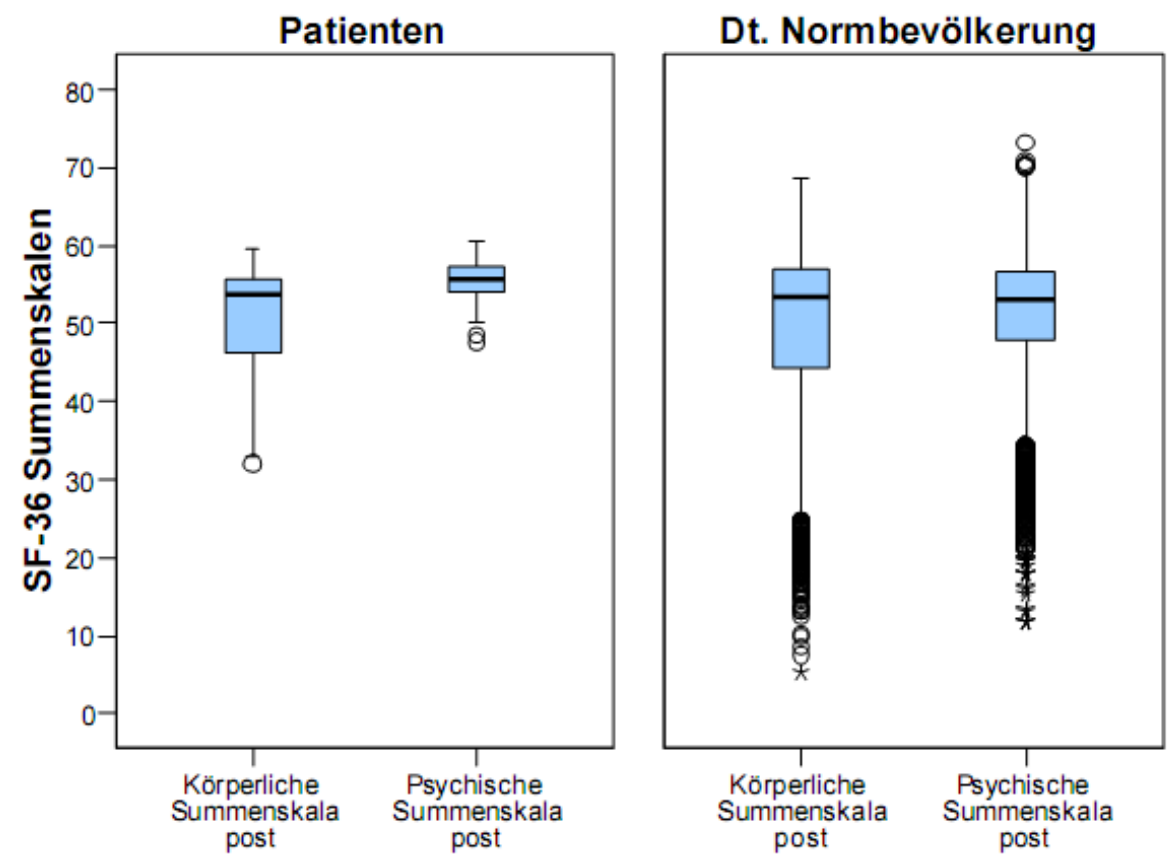

Abb. 25: postoperative Summenskalenwerte Im Vergleich mit der deutschen Normbevölkerung; signifikante Unterschiede in der psychischen Summenskala $(p<0,001)$, $\left({ }^{*}\right.$ - extremer Wert, o - Ausreißer) 
Außerdem wurde die Veränderung der Sub- und Summenskalenwerte des SF-36 ausgewertet. Tabelle 14 zeigt die Veränderung der prä- und postoperativen Punktwerte des SF-36. Die größten Veränderungen der Punktwerte konnten in den Subskalen „Körperliche Schmerzen“ (+ 15 Punkte), „Allgemeine Gesundheit“ (+ 27 Punkte) und der „Körperlichen Summenskala“ (+ 7) nachgewiesen werden.

\begin{tabular}{|c|c|c|c|c|c|c|c|c|}
\hline & \multirow[b]{2}{*}{$\mathrm{N}$} & \multirow[b]{2}{*}{ Mittelwert } & \multirow{2}{*}{$\begin{array}{l}\text { Stand ardabw } \\
\text { eichung }\end{array}$} & \multirow[b]{2}{*}{ Minimum } & \multirow[b]{2}{*}{ Maximum } & \multicolumn{3}{|c|}{ Perzentile } \\
\hline & & & & & & 25. & 50. (Median) & 75. \\
\hline Veränderung KÖFU & 31 & 3,2258 & 15,19585 & $-30,00$ & 35,00 & $-5,0000$ & 5,0000 & 10,0000 \\
\hline Veränderung KÖRO & 31 & 4,8387 & 25,34705 & $-50,00$ & 50,00 &, 0000 &, 0000 & .0000 \\
\hline Veränderung KÖSC & 31 & 14,8710 & 17,00342 & $-10,00$ & 49,00 & 1,0000 & 12,0000 & 28,0000 \\
\hline Veränderung AGES & 31 & 27,3226 & 15,60638 & $-2,00$ & 58,00 & 15,0000 & 27,0000 & 37,0000 \\
\hline Veränderung VITA & 31 & 4,3548 & 8,03554 & $-10,00$ & 20,00 &, 0000 & 5,0000 & 10,0000 \\
\hline Veränderung SOFU & 31 & 3,2258 & 12,48655 & $-25,00$ & 25,00 &, 0000 &, 0000 & 12,5000 \\
\hline Veränderung EMRO & 31 & $-5,3763$ & 15,14565 & $-66,67$ &, 00 &, 0000 &, 0000 &, 0000 \\
\hline Veränderung PSYC & 31 & 1,0323 & 6,52934 & $-20,00$ & 16,00 &, 0000 &, 0000 & 4,0000 \\
\hline Veränderung KSK & 31 & 6,7203 & 6,77423 & $-10,26$ & 21,18 & 3,0356 & 7,3011 & 10,3351 \\
\hline Veränderung PSK & 31 &,- 8773 & 4,15890 & $-14,09$ & 6,62 & $-2,4493$ &,- 6096 & 1,3526 \\
\hline
\end{tabular}

Tab. 14: Veränderung der prä- und postoperative Punktwerte der Subskalen

Des Weiteren wurde geprüft, ob eine Korrelation zwischen Body-Maß-Index, Defektgröße, Patientenalter zu OP und Zeitraum zwischen Ereignis und OP und den Skalenwerten des SF-36 nachzuweisen ist. Die Berechnungen zeigten einen signifikanten Koeffizienten zwischen BMI und der Veränderung in der Skalen „Körperliche Funktionsfähigkeit“ ( $p=0,013$, Korrelation nach Pearson) sowie in der Skala „Emotionale Rollenfunktion“ ( $p=0,01$, Korrelation nach Pearson). Abbildung 26 (S. 45) stellt den Zusammenhang der Veränderung der „Körperlichen Funktionsfähigkeit“ (KÖFU) und dem BMI graphisch dar. Die Regressionsgerade zeigt einen linearen Zusammenhang der Werte in der Punktwolke. Ein höherer BMI korreliert in dem Sinne mit einer geringeren Veränderung bzw. mit einer Verschlechterung in der Skala „Körperliche Rollenfunktion“. Der Korrelationskoeffizient betrug 0,194. (Der Korrelationskoeffizient ist ein dimensionsloses Maß für den Grad eines linearen Zusammenhangs. Sein Wert kann zwischen -1 und 1 liegen. Bei einem Wert von 0 besteht keine Korrelation.) Abbildung 27 (S. 45) veranschaulicht die Korrelation der Punktwerte der Veränderung in der Skala „Emotionale Rollenfunktion“ und BMI. Ein höherer BMI korreliert mit einer Verschlechterung der Skalenwerte EMRO. Bei allen anderen Subskalen wurden keine signifikanten Korrelationen mit dem BMI festgestellt. 


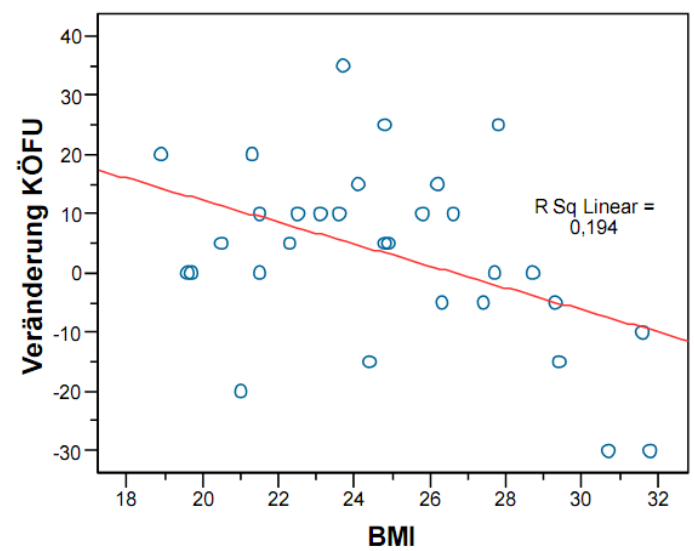

Abb. 26: Korrelation der Werte KÖFU und des BMI signifikanter Koeffizient zwischen BMl und der Veränderung in der Skala KÖFU, ( $p=0,013$, Korrelation nach Pearson)

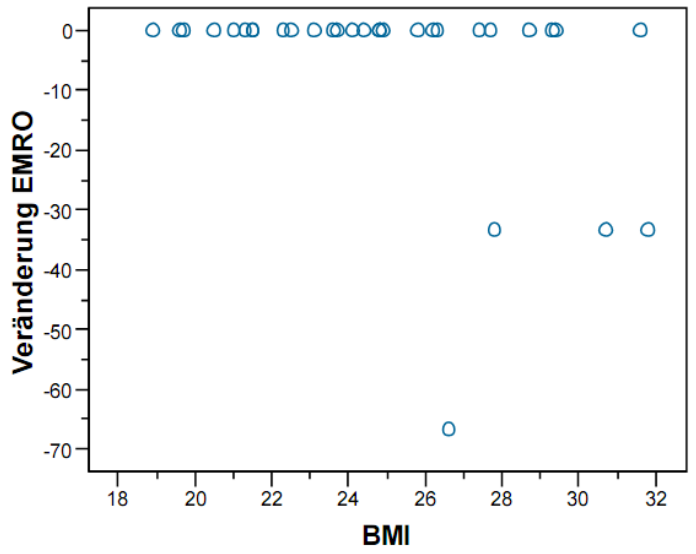

Abb. 27: Korrelation der Werte EMRO mit den Werten des BMI Signifikanter Koeffizient zwischen BMI und der Veränderung der Skala EMRO, $(p=0,01$, Korrelation nach Pearson) 
Abbildung 28 stellt den Zusammenhang zwischen der Veränderung des „Psychischen Wohlbefindens“ und der Defektgröße dar. Es besteht eine signifikante Korrelation der Skalenwerte des SF-36 zwischen der Defektgröße und dem „Psychischen Wohlbefinden“ ( $p=0,036$, Rangkorrelation nach Spearman).

Bei den Variablen Patientenalter zum Operationszeitpunkt und der Zeit zwischen Trauma bzw. Ereignis und OP konnten keine signifikanten Korrelationen festgestellt werden.

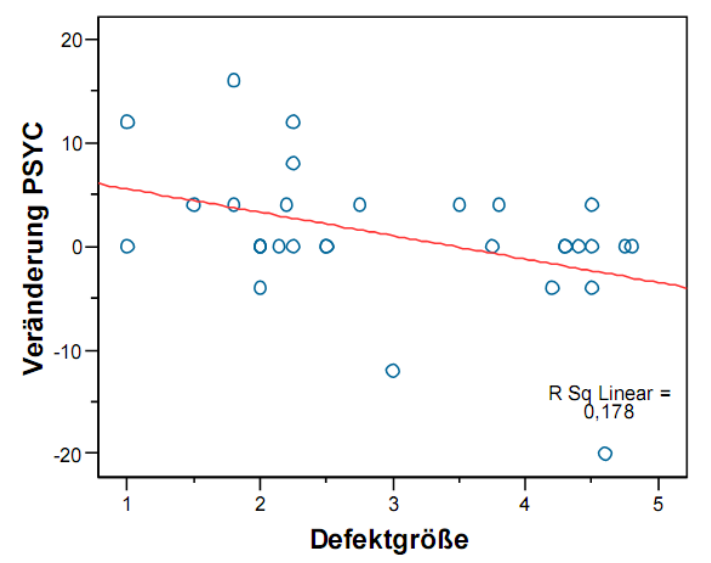

Abb. 28: Korrelation der Werte PSYC mit der Defektgröße Signifikante Korrelation, ( $p=0,036$, Rangkorrelation nach Spearman)

Zusätzlich wurden Daten zur Veränderung des Gesundheitszustandes im Vergleich zu vor einem Jahr erfasst (Abb. 29). Präoperativ lagen die Werte ausschließlich im Bereich von etwas schlechter als vor einem Jahr $(n=11)$ und viel schlechter als vor einem Jahr $(n=19)$. Postoperativ verteilen sich die Patienten über die Bereiche: viel besser als vor einem Jahr $(n=12)$, etwas besser als vor einem Jahr $(n=7)$, annähernd ähnlich wie vor einem Jahr $(n=11)$ und etwas schlechter als vor einem Jahr $(n=1)$.

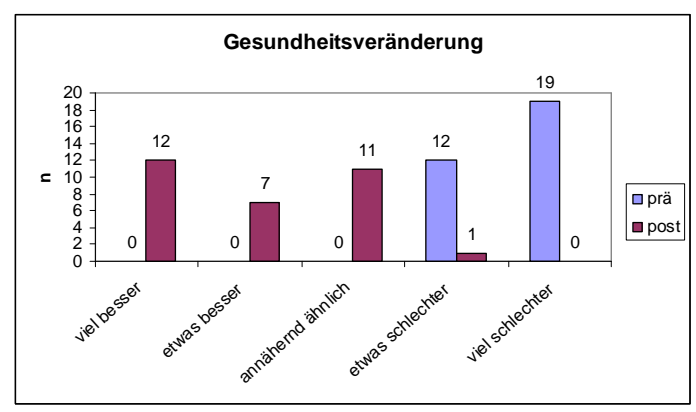

Abb. 29: Gesundheitsveränderung 


\subsection{ERGEBNISSE DES IKDC-FRAGEBOGENS}

Die Tabelle 15 und Abbildung 30 geben einen Überblick über die jeweils prä- und postoperativ erreichten Punktwerte des IKDC-Fragebogens. Ein höherer Wert entspricht einer Verbesserung und ein niedrigerer Wert einer Verschlechterung der Kniegelenksfunktion. Der IKDC-Wert ist postoperativ signifikant höher als präoperativ $(p<0,001, t-T e s t$ für verbundene Stichproben).

\begin{tabular}{|c|c|c|c|c|c|c|c|c|}
\hline & \multirow[b]{2}{*}{$\mathrm{N}$} & \multirow[b]{2}{*}{ Mittelwert } & \multirow{2}{*}{$\begin{array}{l}\text { Standardabw } \\
\text { eichung }\end{array}$} & \multirow[b]{2}{*}{ Minimum } & \multirow[b]{2}{*}{ Maximum } & \multicolumn{3}{|c|}{ Perzentile } \\
\hline & & & & & & 25. & 50. (Median) & 75. \\
\hline IKDC präoperativ & 31 & 60,5481 & 12,52283 & 35,63 & 81,61 & 52,8700 & 60,9200 & 70,1100 \\
\hline IKDC postoperativ & 31 & 76,1584 & 16,60120 & 34,48 & 100,00 & 64,3700 & 79,3100 & 87,3600 \\
\hline
\end{tabular}

Tab. 15: prä- und postoperative Punktwerte des IKDC

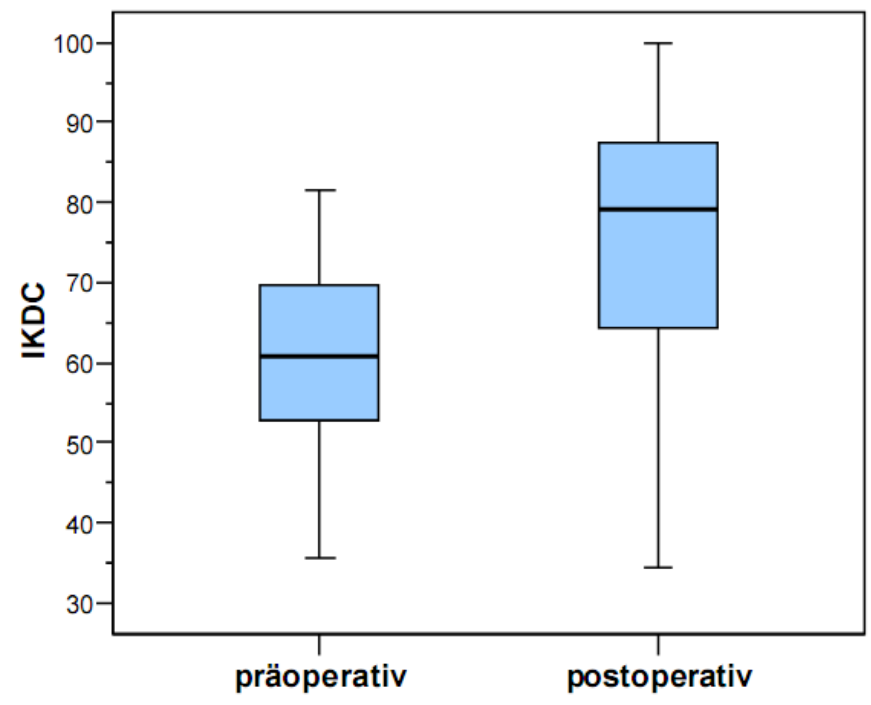

Abb. 30: Vergleich der prä- und postoperativen Punktwerte des IKDC ( $p<0,001, t-T e s t)$ 
Abbildung 31 stellt die Veränderung zwischen den prä- und postoperativen Punktwerten des IKDC dar. In 26 Fällen gab es eine Verbesserung. In fünf Fällen lag eine Verschlechterung vor. Die höchste Punktwertveränderung betrug 58,62. Die geringste Veränderung betrug 0 . Durchschnittlich kam es zu einer Punktwertänderung um plus 15,6 Punkte, wobei die niedrigste Veränderung bei einem Wert von -6,9 (negatives Ausmaß) lag.

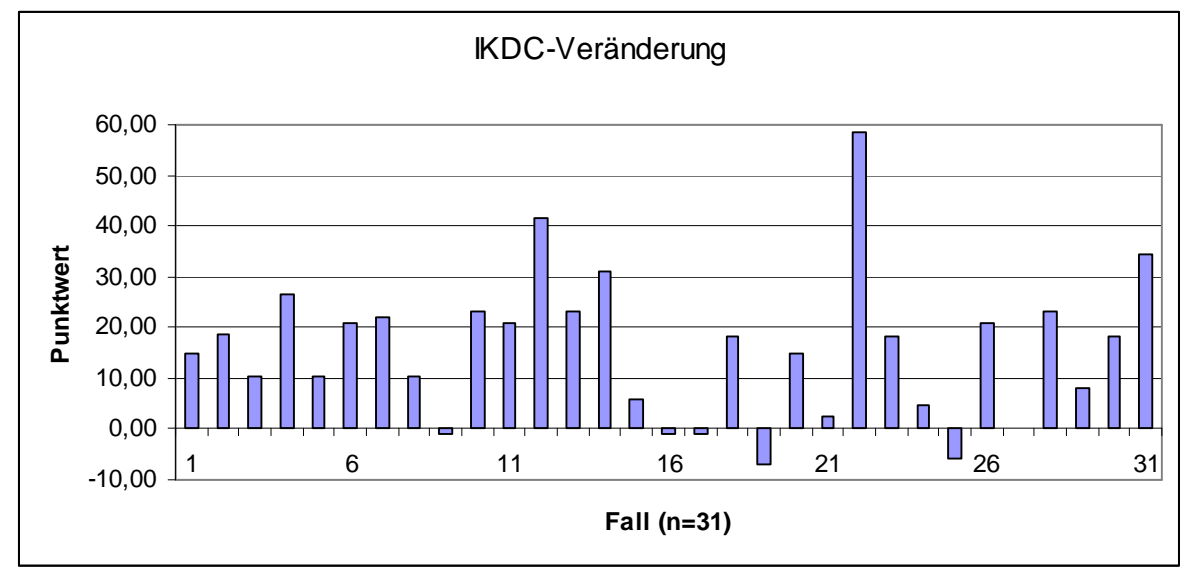

Abb. 31: Veränderung des IKDC-Wertes je nach Fall

Abbildung 32 stellt den prä- und postoperativen IKDC-Gruppengrad dar. Präoperativ wurden elf Patienten in den Gruppengrad C, 20 Patienten in den Gruppengrad D und kein Patient den Gruppengrad A oder B eingestuft. Postoperativ wurden 19 Patienten in Gruppengrad A, elf Patienten in den Gruppengrad B, ein Patient in den Gruppengrad $C$ und kein Patient in Gruppegrad D eingestuft.

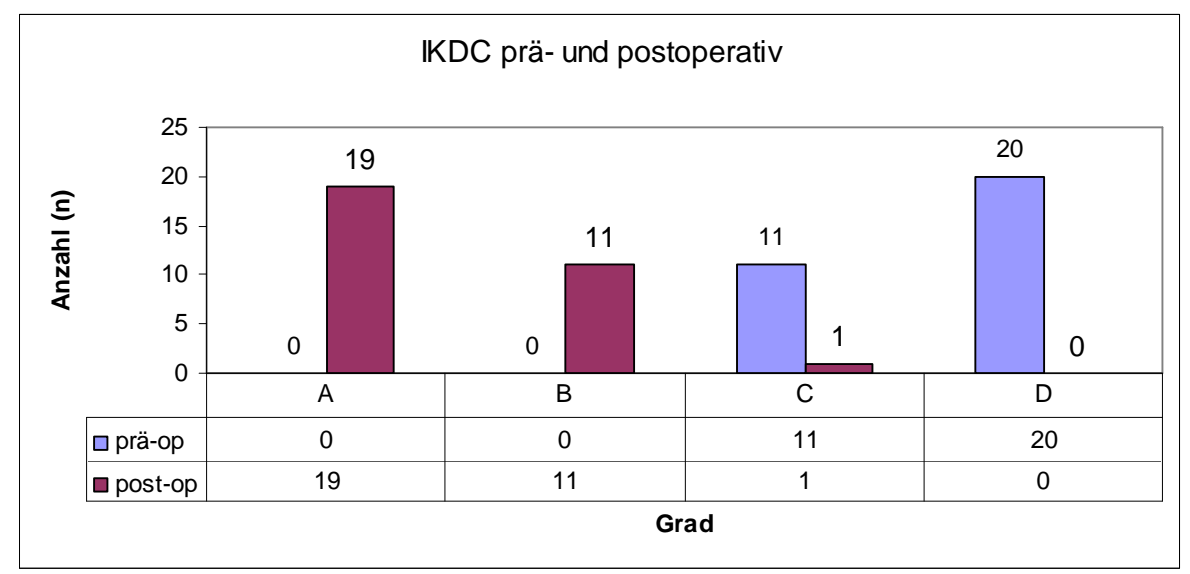

Abb. 32: $\quad$ prä- und postoperative Gruppengrade des IKDC

A - bester Gruppengrad,

D - schlechtester Gruppengrad 
Des Weiteren konnte eine signifikante Korrelation zwischen dem BMI und der Veränderung des IKDC-Wertes festgestellt werden $(p=0,025$, Korrelation nach Pearson, Abb. 33). Ein höherer BMI korreliert mit einer geringeren Veränderung des IKDC-Wertes. Im Vergleich der Veränderung des Mittelwertes der IKDC-Werte zweier BMI-Gruppen (BMI größer bzw. kleiner $25 \mathrm{~kg} / \mathrm{m}^{2}$ ) zeigt sich, dass bei Patienten mit einem geringeren BMI eine größere Veränderung des Mittelwertes vorliegt (Tab. 16). Die Variablen Defektgröße, die Zeit zwischen Ereignis/Trauma und OP, sowie das Lebensalter der Patienten zum Operationszeitpunkt zeigen keine signifikante Korrelation zur Veränderung des IKDC-Punktwertes.

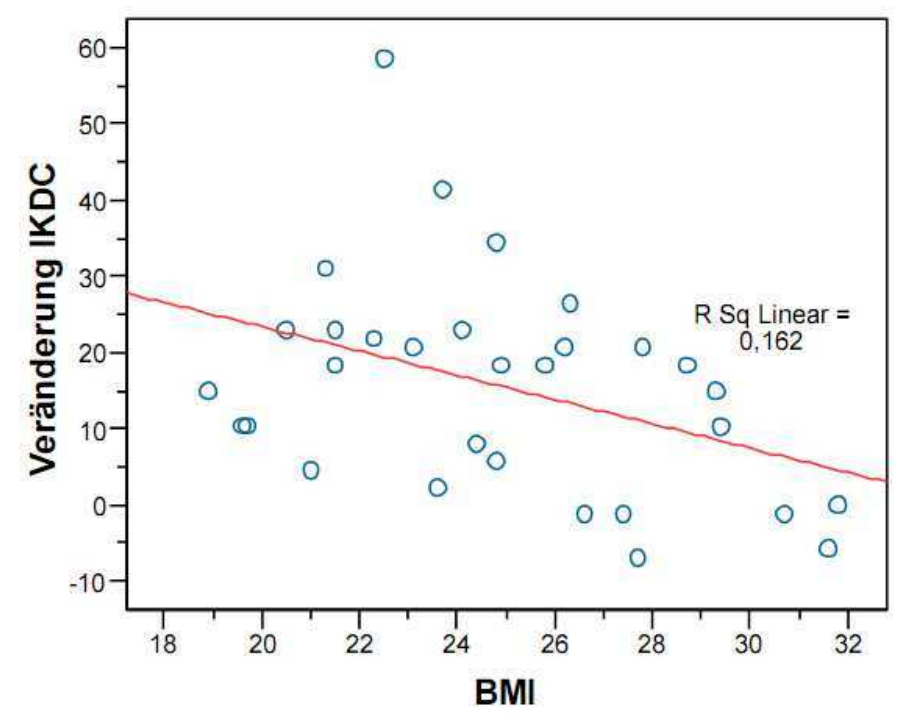

Abb. 33: $\quad$ Korrelation zwischen IKDC-Veränderung und BMI $(p=0,025$, Korrelation nach Pearson)

\begin{tabular}{|c|c|c|c|c|c|c|c|c|c|}
\hline \multirow[b]{2}{*}{ BMI } & & \multirow[b]{2}{*}{$\mathrm{N}$} & \multirow[b]{2}{*}{ Mittelwert } & \multirow{2}{*}{$\begin{array}{l}\text { Standardabw } \\
\text { eichung }\end{array}$} & \multirow[b]{2}{*}{ Minimum } & \multirow[b]{2}{*}{ Maximum } & \multicolumn{3}{|c|}{ Perzentile } \\
\hline & & & & & & & 25. & 50. (Median) & 75. \\
\hline unter 25 & Veränderung IKDC & 18 & 20,56 & 14,18 & 2,30 & 58,62 & 9,77 & 19,54 & 25,00 \\
\hline ab 25 & Veränderung IKDC & 13 & 8,75 & 11,73 & $-6,90$ & 26,44 & $-1,15$ & 10,34 & 19,54 \\
\hline
\end{tabular}

Tab. 16: Veränderung der Mittelwerte des IKDC zwischen zwei BMI-Gruppen 


\subsection{ERGEBNISSE DES ICRS-BEURTEILUNGSBOGENS}

Zur Erhebung des ICRS-Wertes lagen für die präoperativen Werte alle Daten ( $n=31)$ vor. Postoperativ konnten 28 Fälle ausgewertet werden. In drei Fällen waren die Daten entweder unvollständig erhoben oder es lag keine standardisierte Auswertung vor.

Abbildung 34 stellt die Verteilung der Bewertungen zur Knorpelbeschaffenheit jeweils prä- und postoperativ dar. Präoperativ lagen in 21 von 31 Fällen abnormale Knorpelverhältnisse (Grad III, 7 bis 4 Punkte) und in 10 Fällen sehr abnormale Knorpelverhältnisse (Grad IV, 3 bis 1 Punkt) vor. Normale bzw. annähernd normale Knorpelverhältnisse zeigten sich präoperativ in diesem Kollektiv nicht.

Postoperativ stellten sich 12 der 28 Fälle als normal (Grad I, 12 Punkte), 14 Fälle als fast normal (Grad II, 11 bis 8 Punkte), zwei Fälle als abnormal (Grad III, 7 bis 4 Punkte) und kein Fall als sehr abnormal (Grad IV, 3 bis 1 Punkt) dar.

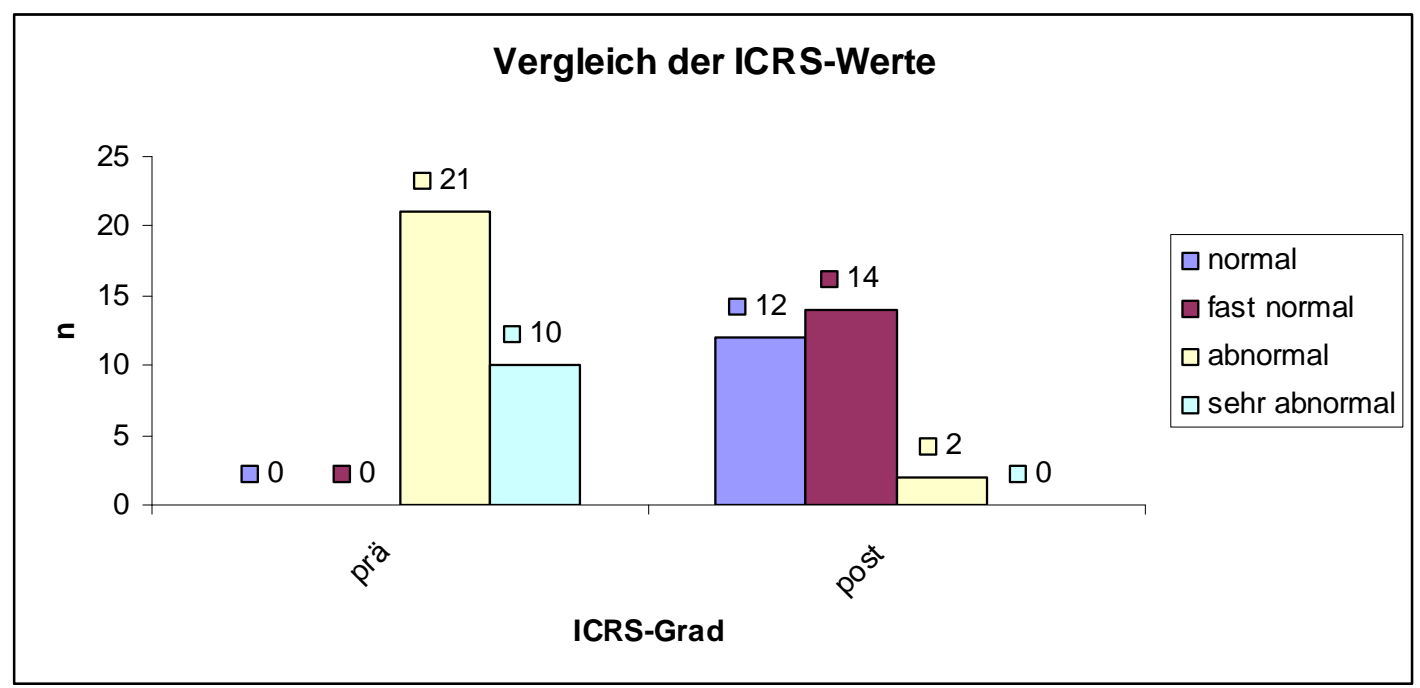

Abb. 34: Vergleich prä- und postoperativer ICRS-Wert 


\section{DISKUSSION}

Um die Lebensqualität nach Knorpel-Knochen-Transplantation zu objektivieren, existieren standardisierte Messinstrumente. Dazu zählt der zuvor beschriebene ICRSEvaluierungs-Fragebogen (Smith et al. 2005) mit dem zugehörigen Teil des SF-36Fragebogens (Bullinger und Kirchberger 1998).

Gesundheitsbezogene Lebensqualität bezeichnet ein multidimensionales psychologisches Konstrukt, bestehend aus mindestens vier Komponenten: psychisches Befinden, körperliche Verfassung, soziale Beziehungen und funktionale Kompetenz (Bullinger und Kirchberger 1998). Der Begriff Lebensqualität beinhaltet zahlreiche Lebensbereiche mit gesundheitlichen, sozialen, beruflichen und ökonomischen Aspekten. Gute Lebensqualität bedeutet, die Möglichkeit zu besitzen, ein Leben zu führen wie ein Gesunder mit normalen Organen ohne jegliche Einschränkungen oder Belastungen. Wissenschaftlich betrachtet ist Lebensqualität eine relative Größe und abhängig von verschiedenen Faktoren wie beispielsweise den Vorstellungen im jeweiligen Kulturkreis oder der entsprechenden Altersgruppe (Schlitt 2006). Hinsichtlich des SF-36 existieren Normwerte für die entsprechende Population. Daher ist es möglich, Vergleiche der Studienergebnisse mit den 1994 erstellten Normdaten der deutschen Bevölkerung (Deutsche Normstichprobe, Durchschnittsalter 47,7 Jahre, davon $56,6 \%$ Frauen) anzustellen (Bullinger und Kirchberger 1998). Studienergebnisse zum Lebensqualitätsgewinn nach Knorpel-Knochen-Transplantation mit entsprechendem Nachuntersuchungsintervall wurden bisher nicht publiziert.

Die Auswertung des SF-36-Fragebogens zeigt signifikante Unterschiede zwischen den prä- und postoperativen Werten der Subskalen „Körperliche Schmerzen“ ( $p<0,001)$, „Allgemeine Gesundheitswahrnehmung" $(p<0,001)$ und der "Vitalität" $(p=0,008)$ (Abb. 22, S. 41). In der Betrachtung der Summenskalen zeigt sich des Weiteren eine signifikante Veränderung der Werte der „Körperlichen Summenskala“ $(p<0,001)$ (Abb. 23, S. 41). Durchschnittlich sieben Jahre nach operativer Therapie war demnach eine deutliche Schmerzreduktion, eine Verbesserung des subjektiven Gesundheitsgefühles und des Vitalitätsempfindens der Patienten zu verzeichnen. Ingesamt kam es postoperativ zur Reduktion der somatischen Beschwerden. In diesem Patientenkollektiv lässt sich jedoch keine Verbesserung der Werte der „Körperlichen Funktionsfähigkeit“, „Körperlichen Rollenfunktion“, „Sozialen Funktionsfähig- 
keit“, „Emotionalen Rollenfunktion“ oder der „Psychischen Summenskala“ nach operativer Therapie nachweisen $(p>0,05)$. Ursächlich liegt dem zugrunde, dass präoperativ bereits nur geringe Einschränkungen in den Subskalen „Körperliche Funktionsfähigkeit“, „Soziale Funktionsfähigkeit“ und in der „Psychischen Summenskala“ vorlagen. In den Subskalen „Körperliche Rollenfunktion“ und "Soziale Rollenfunktion“ lagen präoperativ keinerlei Einschränkungen vor. Dies impliziert, dass das klinische Beschwerdebild mit den genannten Skalenbereichen in diesem Patientenkollektiv initial nicht im direkten Zusammenhang stand. Eine signifikante negative Veränderung der Werte war in keiner der Skalen nachzuweisen.

Daraus lässt sich schlussfolgern, dass durch die postoperative Verbesserung der Werte in den Subskalen „Körperliche Schmerzen“, „Allgemeine Gesundheitswahrnehmung“, „Vitalität“ und der „Körperliche Summenskala“ eine Verbesserung der Lebensqualität erreicht werden konnte. Dies liegt insbesondere in der Reduktion der klinischen Beschwerdesymptomatik (Schmerzreduktion) und in der Verbesserung der subjektiven somatischen Patientensituation begründet.

Aufgrund des Studiendesigns lagen prä- und postoperativ nur absolute Werte vor. Eine Verlaufsbeurteilung zu mehr als zwei Zeitpunkten (Zeitpunkt $t_{0}$ und $t_{1}$ ) konnte daher nicht vorgenommen werden. Zur Einschätzung der Skalenwertveränderung wurde zusätzlich das Ausmaß der Punktwertveränderung untersucht (Tab. 14, S. 44). Die Ergebnisse unterstreichen den positiven Effekt auf die Veränderung der somatischen Beschwerden (Subskalen „Körperliche Schmerzen“, „Allgemeine Gesundheitswahrnehmung“ und „Körperliche Summenskala“). Hier zeigt sich in den 8 Subskalen und der "Körperlichen Summenskala“ eine positive Veränderung, wobei die größte positive Veränderung in der Subskala „Körperliche Schmerzen“ zu finden war. Im Vergleich der postoperativen Werte mit der deutschen Normstichprobe ließen sich signifikant bessere Skalenwerte in den Subskalen „Allgemeine Gesundheitswahrnehmung“ ( $p=0,046)$, „Vitalität“ ( $p<0,001)$ und „Psychisches Wohlbefinden“ $(p=0,040)$ nachweisen (Abb. 24, S. 43). Im Vergleich zur deutschen Normstichprobe ließen sich des Weiteren signifikant bessere Skalenwerte in der "Psychischen Summenskala“ $(p<0,001)$ nachweisen (Abb. 25, S. 43). Hier zeigt sich im Patientenkollektiv eine geringere Spannweite der Skalenwerte mit einer Konzentrierung der Mittelwerte auf das Niveau der Mittelwerte der deutschen Normstichprobe. Die Skalenwerte der „Körperlichen Summenskala" entsprechen nahezu denen der deutschen Normstichprobe. 
Die postoperativen Werte der Subskalen „Körperliche Funktionsfähigkeit“, „Körperliche Rollenfunktion“, „Soziale Rollenfunktion“ und „Emotionale Rollenfunktion“ sowie der „Körperlichen Summenskala“ entsprechen ebenfalls den Werten der gesunden deutschen Normbevölkerung und können somit als normal eingestuft werden. Es lässt sich schlussfolgern, dass im Vergleich zur Deutschen Normstichprobe im Patientenkollektiv postoperativ eine hohe patienteneigene Gesundheitswahrnehmung, ein höheres Vitalitätsempfinden und ein besserer psychischen Status bestehen. Die postoperativen Werte für „Körperliche Funktionsfähigkeit“, „Körperliche Rollenfunktion“, „Soziale Rollenfunktion“, „Emotionale Rollenfunktion“ und der „Körperlichen Summenskala" korrelieren mit den Normwerten und sind demnach als positives Ergebnis zu werten.

Zu berücksichtigen ist, dass das Durchschnittsalter im Patientenkollektiv bei 35 Jahren lag und bekanntermaßen signifikante Unterschiede der Lebensqualität in Abhängigkeit des Alters in der deutschen Normpopulation bestehen. Besonders ausgeprägt sind die Unterschiede in den Skalen „Körperliche Funktionsfähigkeit“, „Körperliche Rollenfunktion“ und „Körperliche Schmerzen“. Bei jüngeren Befragten ist in der Normpopulation eine höhere Lebensqualität nachzuweisen (Bullinger und Kirchberger 1998). Daher wurde zusätzlich der Vergleich der Studiendaten mit den altersgruppenspezifischen Normwerten der deutschen Bevölkerung (Altersgruppe der 31 40jährigen) durchgeführt (Tab. 17). Im altersgruppenspezifischen Vergleich konnte eine Annäherung der Werte an die Normbevölkerung gezeigt werden. Dies belegt die Korrelation der postoperativen Werte mit denen der entsprechenden Altersgruppe der Normbevölkerung und bestätigt ein positives Ergebnis.

\begin{tabular}{|lcc|}
\hline Subskala & Dt. Normstichprobe & Studie \\
& \multicolumn{2}{c|}{ Mittelwert } \\
Körperliche Funktionsfähigkeit & 93,6 & 84,58 \\
Körperliche Rollenfunktion & 90,16 & 87,1 \\
Körperliche Schmerzen & 86,84 & 79,1 \\
Allgemeine Gesundheitswahrnehmung & 74,23 & 74,68 \\
Vitalität & 65,66 & 80,65 \\
Soziale Funktionsfähigkeit & 89,54 & 88,71 \\
Emotionale Rollenfunktion & 91,33 & 94,62 \\
Psychisches Wohlbefinden & 74,31 & 79,61 \\
\hline
\end{tabular}

Tab. 17: $\quad$ Vergleich der Mittelwerte der Altersgruppe der 31- 40jährigen mit dem Patientenkollektiv 
Zusätzlich gehen It. Handanweisung des SF-36 die unterschiedlichen Bundeslandeffekte, die Unterschiede zwischen Ost- und Westdeutschland, sowie die geschlechtlichen Unterschiede in die Auswertung ein. Aufgrund des kleinen Patientenkollektivs wurden diese Faktoren in den Berechnungen vernachlässigt.

Die Prüfung auf Korrelation zwischen Body-Maß-Index und der Veränderung der Skalenwerte des SF-36 zeigte einen signifikanten Zusammenhang zwischen dem BMI und den Skalen „Körperliche Funktionsfähigkeit“ $(p=0,013)$ und „Emotionale Rollenfunktion“ $(p=0,01)$. Mit zunehmendem BMI nimmt die Veränderung der „Körperlichen Funktionsfähigkeit“ linear ab (Abb. 26, S. 45). Des Weiteren zeigt sich in einigen Fällen eine Verschlechterung der „Emotionalen Rollenfunktion“ mit zunehmendem BMI (Abb. 27, S. 45). Daraus lässt sich schlussfolgern, dass ein erhöhter BMI hinsichtlich des postoperativen Ergebnisses einen negativen Einfluss in Hinblick auf die Lebensqualität ausübt.

Die Prüfung auf Korrelation zwischen Defektgröße und der Veränderung der Skalenwerte des SF-36 zeigte eine signifikanten Zusammenhang zwischen der Defektgröße und der Veränderung des „Psychischen Wohlbefindens“ ( $p=0,036)$. Mit zunehmender Defektgröße nimmt die Veränderung des „Psychischen Wohlbefindens“ linear ab (Abb. 28, S. 46). Daraus lässt sich schlussfolgern, dass die Knorpel-KnochenTransplantation bei größeren Defekten mit einer Verschlechterung des psychischen Wohlbefindens einher geht. Denkbare Ursachen sind hier Einschränkungen der Kniegelenksfunktion eines so enormen Ausmaßes, dass nach körperlicher auch die psychische Einschränkung folgt bzw. dass neben dem Knorpelschaden diverse Begleitverletzungen vorlagen, welche den Therapieeffekt im negativen Sinne beeinflussen. Zusätzlich war im Vergleich der Werte zur Beurteilung des Gesundheitszustandes (Veränderung im Vergleich mit einem Jahr zuvor) eine deutliche Verbesserung zu verzeichnen. Postoperativ bezeichnete keiner der Patienten den Gesundheitszustand als etwas bzw. viel schlechter als ein Jahr zuvor.

Die Ergebnisse zeigen einen signifikant positiven Zusammenhang zwischen der Verbesserung der Lebensqualität und der Skalen „Körperliche Schmerzen“, „Allgemeine Gesundheitswahrnehmung“ und „Vitalität". Des Weiteren besteht eine signifikante Verbesserung der „Allgemeinen Gesundheitswahrnehmung“, „Vitalität“ und „Psychischen Rollenfunktion“ im Vergleich zur deutschen Normbevölkerung. Body-MaßIndex und Defektgröße stehen unmittelbar in Zusammenhang mit der Veränderung 
der Lebensqualität in diesem Patientenkollektiv. Patientenalter zum Operationszeitpunkt sowie Zeit zwischen Trauma bzw. Ereignis und OP konnten keine signifikanten Korrelationen festgestellt werden. Der Verlust des Einflusses des Patientenalters auf das postoperative Ergebnis erklärt sich durch die strenge Indikationsstellung. Ab einem grenzwertigen Alter sind degenerative Veränderungen und Begleitpathologien präexistent. Diese Patienten wurden bei der Indikationsstellung ausgeschlossen.

Hinsichtlich der Beurteilung und Objektivierung der Kniegelenkfunktion zeigte die Auswertung des IKDC-Fragebogens eine signifikante Verbesserung der postoperativen Werte $(p<0,001)$ (Abb. 30, S. 47). Diese geht mit einer verbesserten subjektiven Kniegelenkfunktion einher und beruht auf einem verbesserten objektiven klinischen Untersuchungsbefund. Nach operativer Therapie besteht demnach auch langfristig eine deutliche Verbesserung der Kniegelenkfunktion in diesem Patientenkollektiv. Aufgrund der geringen Fallzahl wurde zusätzlich die Veränderung der einzelnen Fälle untersucht. Hier zeigt sich in 26 Fällen eine deutliche Verbesserung der Werte (Abb. 31, S.48). Des Weiteren konnte eine Verbesserung des IKDC-Gruppengrades nachgewiesen werden (Abb. 32, S. 48). Es fand ein Änderung des Gruppengrades von C und $D$ nach $A$ und $B$ statt. Die operative Therapie führte somit zu einer deutlichen Verbesserung des Gruppengrades und somit zu einer Annäherung der Kniegelenkfunktion an den besten Gruppengrad im Sinne eines normwertigen Kniegelenkbefundes.

Vergleichbare Studien mit einer entsprechenden mittel- bis langfristigen Beurteilung des Therapieeffektes unter Nutzung des IKDC-Score wurden beispielsweise von Klinger et al. (2003) und Chow et al. (2004) durchgeführt.

Klinger et al. (2003) untersuchten die Ergebnisse der Knochen-KnorpelTransplantation in Kombination mit der Rekonstruktion des vorderen Kreuzbandes anhand eines Patientenkollektivs von 21 Patienten (Durchschnittsalter 29 Jahre) und einem Nachuntersuchungsintervall von 32 Monaten und länger. Hier zeigte sich postoperativ in $81 \%$ der Fälle ein normaler (24\%) bzw. fast normaler (57\%) IKDCWert.

Chow et al. (2004) führten an 33 Patienten (Durchschnittsalter 44,6 Jahre) in einem Intervall von 45,1 Monaten entsprechende Nachuntersuchungen durch. In 26 Fällen (86\%) wurde die Kniegelenkfunktion anhand des IKDC-Scores als normal $(26,7 \%)$ bzw. fast normal $(60 \%)$ beurteilt. 
Vergleicht man die Ergebnisse der Studie (prä- und postoperativen klinischen Punktwerte des IKDC) mit denen bisheriger Untersuchungen (Chow et al. 2004 und Klinger et al. 2005), korrelieren diese deutlich miteinander (Abb. 35 und Abb. 36).

Hier findet sich ebenfalls eine Änderung des Grades zum nächst besseren Gruppengrad. Dadurch lässt sich herausstellen, dass durch die operative Therapie auch bis 10 Jahre postoperativ eine Verbesserung der Kniegelenkfunktion nachzuweisen ist.

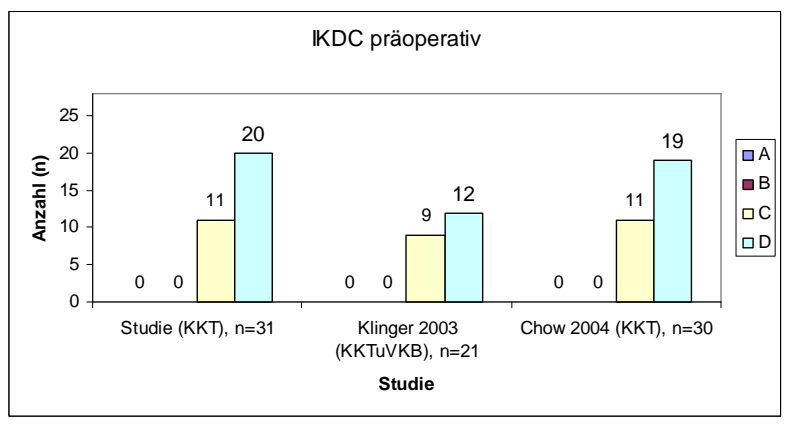

Abb. 35

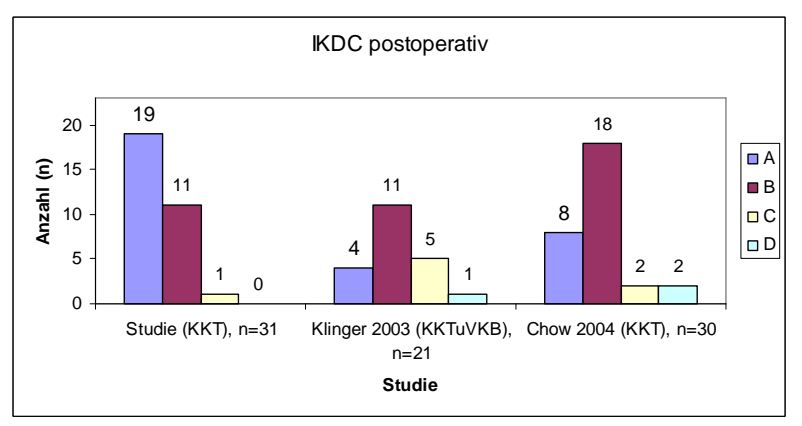

Abb. 35: Vergleich der präoperativen klinischen

Punktwerte des IKDC

Abb. 36: Vergleich der postoperativen klinischen Punktwerte des IKDC

Die Prüfung auf Korrelation zwischen Body-Mass-Index und der Veränderung des IKDC-Wertes zeigte ein linearer Zusammenhang $(p=0,025)$ (Abb. 33, S. 49). Mit zunehmendem BMI nimmt das Ausmaß der Punktwertveränderung ab. D.h. umso höher der BMI, desto geringer ist die Verbesserung der Kniegelenkfunktion postoperativ. Untersucht man 2 verschiedene BMI-Gruppen (größer oder kleiner 25; Grenze Normalgewicht $25 \mathrm{~kg} / \mathrm{m}^{2}$ ), dann zeigt sich in der Gruppe eines BMI von unter 25 eine größere IKDC-Wert-Veränderung im Sinne einer Verbesserung. Daraus lässt sich schlussfolgern, dass ein BMI jenseits der Norm $\left(>25 \mathrm{~kg} / \mathrm{m}^{2}\right)$ einen schlechten Prädiktor hinsichtlich der subjektiven und klinischen Kniegelenksfunktion darstellt. Die Defektgröße, die Zeit zwischen Ereignis bzw. Trauma und OP und das Lebensalter zum 
Operationszeitpunkt zeigen in dieser Studie keine signifikante Korrelation zur Veränderung des IKDC-Punktwertes.

Insbesondere beim Verlust des Einflusses des Patientenalters auf das postoperative Ergebnis ist hier noch mal auf die Indikationsstellung hinzuweisen. Dieser erklärt sich durch den präoperativen Ausschluss bestimmter Altersgruppen für die operative Versorgung.

Anhand der Beurteilung der Knorpelstruktur (ICRS-Beurteilungsbogen) konnte eine deutliche Verbesserung der Knorpelstruktur im Untersuchungsintervall nachgewiesen werden. Postoperativ fand sich in 26 von 28 Fälle ein normaler $(n=12)$ bzw. fast normaler Befund $(n=14)$ nach ICRS.

Vergleichbare Studien mit einer entsprechenden mittel- bis langfristigen Beurteilung des Therapieeffektes unter Nutzung des ICRS-Score wurden von Laprell und Petersen (2001), Koulalis et al. (2004) und Sharpe et al. (2005) durchgeführt. Laprell und Petersen (2001) berichteten über die Ergebnisse nach Knorpel-KnochenTransplantation von 35 Patienten (Durchschnittsalter 26 Jahre) in einem Nachuntersuchungsintervall von durchschnittlich 97 Monaten. Hier zeigte sich postoperativ in der Mehrzahl der Fälle eine normale ( $n=12)$ bzw. fast normale $(n=14)$ Knorpelstruktur.

Koulalis et al. (2004) berichteten über Nachuntersuchungen an 18 Patienten (Durchschnittsalter 36 Jahre) mit einem Nachuntersuchungszeitraum von 27,2 Monaten. Postoperativ fanden sich 12 Fällen mit normaler und sechs Fällen mit fast normaler Knorpelstruktur.

Sharpe et al. (2005) veröffentlichten Studiendaten hinsichtlich der Behandlung der osteochondralen Läsion mit einer Kombination von autologer Chondrozytentransplantation und der Verwendung von osteochondralen Autografts (KnochenKnorpel-Transplantation). 13 Patienten (Durchschnittsalter 42 Jahre) wurden in einem Nachuntersuchungsintervall von 36 Monaten untersucht. Neun Fälle konnten via Arthroskopie gezielt beurteilt werden. Hier zeigte sich in sieben von neun Fällen eine fast normale Knorpelstruktur im Sinne einer guten Integration. Im Vergleich der in der Studie erhobenen Daten mit den Studienergebnissen von Koulalis et al. (2004), Laprell und Petersen (2001) und Sharpe et al. (2005) zeigen sich annähernd ähnliche Ergebnisse (Abb. 37, S. 58). 
Den limitierenden Faktor stellt in diesem Teil der Studie die Nachuntersuchung dar, da sich postoperativ nicht alle Patienten einer Re-Arthroskopie unterzogen. Sofern keine Komplikationen vorlagen, war hier aus ethischen Gesichtspunkten einen erneute Operation nicht indiziert. Daher wurde zur postoperativen Befunderhebung die MRT (nach Studienprotokoll der ICRS) genutzt. Es lässt sich schlussfolgern, dass durch die Knorpel-Knochen-Transplantation auch langfristig eine Verbesserung der Knorpelstruktur zur erwarten ist.

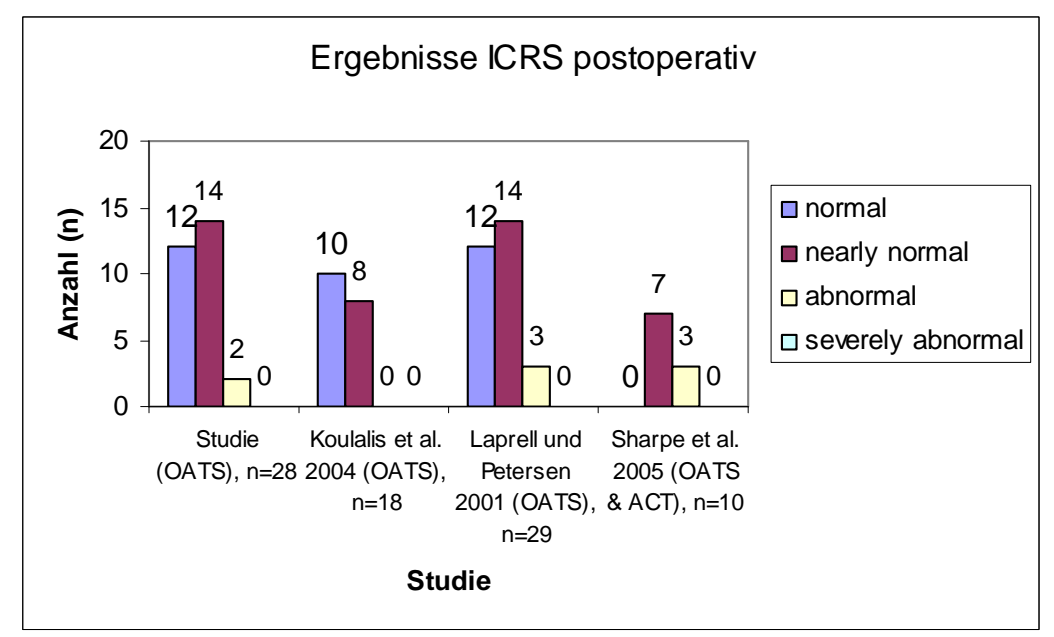

Abb. 37: Vergleich der postoperativen Punktwerte des ICRS

Für das postoperative Ergebnis nach Knorpel-Knochentransplantation sind die optimale Patientenselektion und die korrekte Indikationsstellung entscheidend. Hinsichtlich der Patientenselektion sind Compliance (insbesondere in Hinblick auf die Rehabilitationsphase) und die entsprechenden körperlichen Ressourcen (BMI) entscheidende Faktoren. Die Indikationsstellung richtet sich hauptsächlich nach dem Grad der Schädigung (Grad III und IV nach Outerbridge), nach dem Defektausmaß (optimal nicht mehr als $1-4 \mathrm{~cm}^{2}$ ) und dem Patientenalter (optimal nicht älter als 50 Jahre). Hinsichtlich der Zeit zwischen Ereignis und Operation existiert keine relevante Aussage. In diesem Patientenkollektiv lagen ausschließlich Knorpelschäden im Stadium III $(n=11)$ oder IV $(n=20)$ nach Outerbridge vor.

Die Untersuchungen in diesem Patientenkollektiv belegen, dass ein erhöhter BMI einen die Lebensqualität und Kniegelenkfunktion signifikant beeinflussenden Faktor darstellen kann. Insbesondere verschlechterte sich in der Studie mit zunehmendem 
BMI die Lebensqualität durch Reduktion der Skalen „Körperliche Funktionsfähigkeit“ und „Emotionale Rollenfunktion“.

Des Weiteren zeigen die Studiendaten, dass die Verbesserung der Kniegelenkfunktion umso geringer ausfällt, je größer der vorliegende BMI ist.

Den Schwachpunkt der vorliegenden Studie stellt insbesondere der Einfluss durch die Fremderhebung der Daten dar. Entsprechend dem Handbuch zum SF-36 steht ein Fremderhebungsbogen hinsichtlich der patientenbezogenen Daten zur Verfügung. Voraussetzung dafür ist jedoch eine exakte Dokumentation bzw. Verfügbarkeit der Aktenlage. Aufgrund des damals neuen Operationsverfahrens existiert eine nahezu komplette Dokumentation der einzelnen Fälle. Die Patientenbeurteilung erfolgte anhand der dokumentieren Nachuntersuchungsbefunderhebung und der schriftlich fixierten Befunde stattgehabter apparativer Diagnostik bzw. anhand der Informationen aus Operationsprotokollen. Daher wurden die Einschlusskriterien streng gestellt. Sämtliche Fälle mit fehlender Dokumentation der Nachuntersuchung wurden von der Studie ausgeschlossen.

Des Weiteren ist hervorzuheben, dass aufgrund des kleinen Patientenkollektivs keine allgemein gültige Aussage über die Gesamtbevölkerung gemacht werden darf. Es liegen signifikante Ergebnisse nur hinsichtlich des untersuchten Patientenkollektivs vor. Des Weiteren konnte der postoperative ICRS-Wert nur modifiziert via Auswertung der postoperativ zur Verlaufsbeurteilung durchgeführten MRT-Untersuchungen bestimmt werden, da es aus ethischen Gesichtspunkten keine Indikation zur erneuten Operation gab.

Außerdem waren der Analyse nur absolute Endwerte (prä- und postoperativ) zugänglich. Es konnte somit keine differenzierte Verlaufsbeurteilung zu mehreren Zeitpunkten gemacht werden.

Eindeutige Stärken der vorliegenden Studie sind die benutzten standardisierten Testverfahren (ICRS-Beurteilungspaket, MRT-Protokoll, standardisiertes Operationsverfahren) sowie die Durchführung der Operation durch einen Operateur. 


\section{ZUSAMMENFASSUNG}

In 10 bis $12 \%$ der Gesamtbevölkerung sind Knorpelschäden existent (Sellards et al. 2002). Es ist bekannt, dass sich zerstörter Knorpel nicht mehr aufbauen kann (Hunter 1995). Daher zählt die Behandlung von Knorpelschäden heute grundlegend zum Spektrum der Orthopädie und Unfallchirurgie. Beim Vorliegen von Knorpelschäden Grad III oder Grad IV nach Outerbridge kommt u. a. als operatives Verfahren die autologe Knorpel-Knochen-Transplantation zum Einsatz (Kish et al. 1999). Dabei werden osteochondrale Stanzzylinder aus unbelasteten Gelenkbezirken entnommen und in den defekten Teil einbracht (Hangody et al. 2004).

Ziel der vorliegenden Studie war die Ermittlung des klinischen LangzeitTherapieeffektes der Knorpel-Knochen-Transplantation. Mit der Hypothese, dass im Mittel 7 Jahre postoperativ eine signifikante Verbesserung von Lebensqualität, Gelenkfunktion und Knorpelstruktur vorliegt, wurde der Einfluss von Alter, BMI, Defektgröße sowie Zeit zwischen Ereignis und OP untersucht.

Aus einem Gesamtkollektiv von insgesamt 38 operierten Patienten (Durchschnittsalter 35 Lebensjahre, 21 Männer, 10 Frauen) wurden 31 Patienten hinsichtlich der allgemeinen Lebensqualität (SF-36), Gelenkfunktion (IKDC) und des Status der Knorpelstruktur (ICRS) nachuntersucht (durchschnittlicher Nachuntersuchungszeitraum 7 Jahre, 4,4 - 9,3 Jahre). Der mittlere BMI belief sich zum OP-Zeitpunkt auf $24,89 \mathrm{~kg} / \mathrm{m}^{2}\left(18,9-31,8 \mathrm{~kg} / \mathrm{m}^{2}\right)$. Im Mittel lagen 53,26 Tage zwischen Erstereignis und Operation (7 - 140 Tage). Behandelt wurden monofokale Knorpelschäden im Stadium III $(n=11)$ oder IV $(n=20)$ nach Outerbridge. Die mittlere Defektgröße betrug nach durchgeführtem Debridement $3 \mathrm{~cm}^{2}\left(1-4,8 \mathrm{~cm}^{2}\right)$ Zur Therapie wurde die Knorpel-Knochen-Transplantation angewandt. Sämtliche Eingriffe wurden durch einen Operateur durchgeführt. Die Datenerhebung erfolgte anhand der Aktenlage.

Als Messinstrument diente der ICRS-Evaluierungs-Fragebogen. Dieser wird heute als Standardinstrument angesehen und gilt als ein statistisch sicherer Test mit entsprechender Reliabilität und reproduzierbaren Ergebnissen (Smith et al. 2005). Es ist bekannt, das das makroskopische Erscheinungsbild des Knorpels (sog. "Cartilage Repair Assessment") nicht generell mit dem klinischen Beschwerdebild korreliert, daher ist eine Kombination der einzelnen Fragebögen (SF-36, IKDC, ICRS) nicht valide (Smith et al. 2005). Dementsprechend war es notwendig, die einzelnen Teile 
des Fragebogens separat zu betrachten und auszuwerten. Die Datenauswertung erfolgte mittels SPSS.

Insbesondere Studienergebnisse zum Lebensqualitätsgewinn nach KnorpelKnochen-Transplantation mit entsprechendem Nachuntersuchungsintervall unter Nutzung des SF-36-Fragebogens sind aktuell nicht publiziert worden.

Bisher wurde der SF-36 in Studien mit orthopädischen bzw. unfallchirurgischen Fragestellungen beispielsweise zur Beurteilung des Lebensqualitätsgewinns im Bereich der Endoprothetik angewandt (Jerosch und Floren 2000).

Die Studiendaten belegen eine Verbesserung der Lebensqualität im Patientenkollektiv. Es konnte eine Schmerzreduktion, Verbesserung des subjektiven Gesundheitsgefühles und des Vitalitätsempfindens nachgewiesen werden. Die postoperativen Werten des SF-36 unterliegen in den Subskalen „Körperliche Schmerzen“ ( $p<0,001)$, „Allgemeine Gesundheitswahrnehmung“ ( $p<0,001)$, „Vitalität“ $(p=0,008)$ und der „Körperlichen Summenskala“ $(p<0,001)$ signifikanten Veränderungen.

Hinsichtlich der Beurteilung der Lebensqualität stellt die Gesundheitsveränderung einen entscheidenden Faktor dar. Der allgemeine Gesundheitszustand zeigt sich postoperativ als verbessert. Postoperativ bezeichnete keiner der Patienten den Gesundheitszustand als etwas bzw. viel schlechter als ein Jahr zuvor. Die Veränderung des Gesundheitszustandes liegt anhand der Interpretation der Studiendaten insbesondere in der Verbesserung der somatischen Beschwerden (signifikante Veränderung der Subskalen „Körperliche Schmerzen“, „Allgemeine Gesundheit“ und „Vitalität“ sowie Verbesserung der Werte der „Körperlichen Summenskala") begründet.

Durch die zusätzlich untersuchte Veränderung der einzelnen Punktwerte konnte in drei Subskalen eine Verbesserung nachgewiesen werden („Körperliche Schmerzen“ (+ 15 Punkte), „Allgemeine Gesundheit“ (+ 27 Punkte) und der „Körperlichen Summenskala“ $(+7))$. Insgesamt resultierte eine Reduktion der somatischen Beschwerden.

Vergleicht man die Skalenwerte mit den Normwerten der deutschen Bevölkerung, lässt sich schlussfolgern, dass im Patientenkollektiv postoperativ eine hohe patienteneigene Gesundheitswahrnehmung, ein hohes Vitalitätsempfinden und ein besserer psychischer Status bestehen. Die postoperativen Werte für die Skalen 
„Körperliche Funktionsfähigkeit“, „Körperliche Rollenfunktion“, „Soziale Rollenfunktion“ und „Emotionale Rollenfunktion“ sowie der „Körperlichen Summenskala" korrelieren mit den deutschen Normwerten und sind demnach als positives Ergebnis zu werten.

Unter Berücksichtigung der Tatsache, dass das Durchschnittsalter im Patientenkollektiv bei 35 Jahren lag und bekanntermaßen signifikante Unterschiede der Lebensqualität in Abhängigkeit des Alters in der deutschen Normpopulation bestehen, zeigt der zusätzlich durchgeführte Vergleich der Studiendaten mit den altersgruppenspezifischen Normwerten der deutschen Bevölkerung insbesondere in den Skalen „Körperliche Funktionsfähigkeit“, „Körperliche Rollenfunktion“ und „Körperliche Schmerzen“ eine Annäherung der Werte an die altersentsprechenden Normwerte. Dies belegt die Korrelation der postoperativen Werte mit den altersentsprechenden Normwerten der Bevölkerung und bestätigt ein positives Ergebnis.

In den weiteren Untersuchungen lässt sich ein signifikanter Zusammenhang zwischen Body-Mass-Index und der Veränderung zweier Subskalenwerte des SF-36 nachweisen („Körperliche Funktionsfähigkeit“ $(p=0,013)$ und „Emotionale Rollenfunktion“ $(p=0,01))$. Mit zunehmendem BMI nimmt die Veränderung der „Körperlichen Funktionsfähigkeit“ linear ab. Des Weiteren zeigt sich in einigen Fällen eine Verschlechterung der „Emotionalen Rollenfunktion“ mit zunehmendem BMI. Daraus lässt sich schlussfolgern, dass ein erhöhter BMI hinsichtlich des postoperativen Ergebnisses einen negativen Einfluss in Hinblick auf die Lebensqualität ausübt.

Ebenso zeigt sich ein signifikanter Zusammenhang zwischen Defektgröße und der Veränderung der Skalenwerte des SF-36 ( $p=0,036)$. Mit zunehmender Defektgröße nimmt die Veränderung des „Psychischen Wohlbefindens“ linear ab. Die KnorpelKnochen-Transplantation geht demnach beim Vorliegen größerer Defekte mit einer Verschlechterung des psychischen Wohlbefindens einher.

Zusammenfassend ist festzustellen, dass durch die Knorpel-KnochenTransplantation auch langfristig eine Verbesserung der patienteneigenen Lebensqualität (deutliche Schmerzreduktion, Verbesserung des subjektiven Gesundheitsgefühles, Verbesserung des Vitalitätsempfindens) zu erzielen ist. Insbesondere der BMI und die Defektgröße üben Einfluss auf die Veränderung der Lebensqualität aus. 
Die weiteren Untersuchungen belegen eine signifikante Verbesserung der Gelenkfunktion. In 26 Fällen zeigt sich eine deutliche Verbesserung der postoperativen Skalenwerte des IKDC $(p<0,001)$. BMI und IKDC-Veränderung korrelieren signifikant miteinander $(p=0,025)$. Ein höherer $B M l$ geht mit einer geringeren Veränderung des IKDC-Wertes einher. Bei Patienten mit einem geringeren BMI zeigt sich eine größere Veränderung des Mittelwertes. Insbesondere der Vergleich der Veränderung des Mittelwertes der IKDC-Werte der BMI-Gruppen größer bzw. kleiner $25 \mathrm{~kg} / \mathrm{m}^{2}$ belegt, dass bei Patienten mit einem geringeren BMI eine größere Veränderung des Mittelwertes vorliegt. Der BMI stellt demnach einen entscheidenden Faktor für die resultierende postoperative Gelenkfunktion dar.

Des Weiteren fand eine Änderung des Gruppengrades von $C$ und $D$ nach $A$ und $B$ statt (wobei $A$ dem besten Gruppengrad entspricht). Die operative Therapie führte somit zu einer deutlichen Verbesserung des Gruppengrades und zu einer Annäherung der Kniegelenkfunktion an den besten Gruppengrad im Sinne eines normwertigen Kniegelenkbefundes. Vergleicht man die Ergebnisse mit denen bisheriger Untersuchungen (Chow et al. 2004 und Klinger et al. 2003), korrelieren diese deutlich miteinander und belegen den effektiven Nutzen der operativen Intervention.

Die Auswertung des ICRS-Beurteilungsbogens zeigt in $68 \%$ der Fälle eine postoperative Verbesserung der Knorpelstruktur im ehemaligen Defektareal. Präoperativ lagen in 31 Fällen abnormale $(n=21)$ bzw. sehr abnormale $(n=10)$ Strukturverhältnisse vor. Postoperativ stellte sich die Knorpelstruktur in zwölf Fällen als normal und in 14 Fällen als nahezu normal dar. In der Zusammenschau mit Ergebnissen vergleichbarer Studien (Koulalis et al. 2004, Laprell und Petersen 2001 und Sharpe et al. 2005) zeigen sich annähernd ähnliche Ergebnisse. Die Studienergebnisse belegen die bestehende Verbesserung der Knorpelstruktur nach stattgehabter operativer Intervention auch im Langzeitverlauf.

Die Ergebnisse bestätigen die in der Literatur bisher publizierten Daten und unterstreichen den therapeutischen Nutzen der Knorpel-Knochen-Transplantation. Die Untersuchungen belegen einen positiven klinischen Therapieeffekt hinsichtlich Lebensqualität (Reduktion somatischer Beschwerden), Gelenkfunktion und Knorpelstruktur (Gelenkflächenkongruenz, Zylinderintegration). 
Hervorzuheben ist insbesondere die Verbesserung der Lebensqualität durch eine gezielte operative Therapie.

Body-Mass-Index und Defektgröße stehen unmittelbar in Zusammenhang mit der Veränderung der Lebensqualität. Mit zunehmendem BMI nimmt die Veränderung der „Körperlichen Funktionsfähigkeit“ linear ab. Des Weiteren nimmt die Veränderung des „Psychischen Wohlbefindens“ mit zunehmender Defektgröße linear ab. Ein BMI jenseits der Norm $\left(>25 \mathrm{~kg} / \mathrm{m}^{2}\right)$ stellt einen schlechten Prädiktor hinsichtlich der subjektiven und klinischen Kniegelenksfunktion dar. Hohe Werte von BMI und Defektgröße sind demnach als negative Prädiktoren für das postoperative Ergebnis aufzufassen.

Patientenselektion und Indikationsstellung sind demnach entscheidend für das klinische Ergebnis. Hinsichtlich der Patientenselektion sind neben dem Alter und der Compliance insbesondere die körperlichen Ressourcen (BMI) und die Defektgrösse entscheidende Faktoren. Je größer der BMI oder der Defekt, desto größer scheint die Wahrscheinlichkeit eines schlechteren Ergebnisses zu sein.

Um jedoch eine allgemeingültige Aussage machen zu können, sind in der Zukunft weitere Studien mit größeren Patientenkollektiven notwendig. Den limitierenden Faktor in der Nachuntersuchung stellt die arthroskopische Beurteilung der Knorpelstruktur dar, da aufgrund ethischer Gesichtspunkte bei beschwerdefreiem Patienten keine Indikation zur Operation besteht. 
7. ANHANG : ICRS-BEURTEILUNGSPAKET

ICRS - CARTILAGE INJURY STANDARD EVALUATION FORM-2000 PATIENTS PART

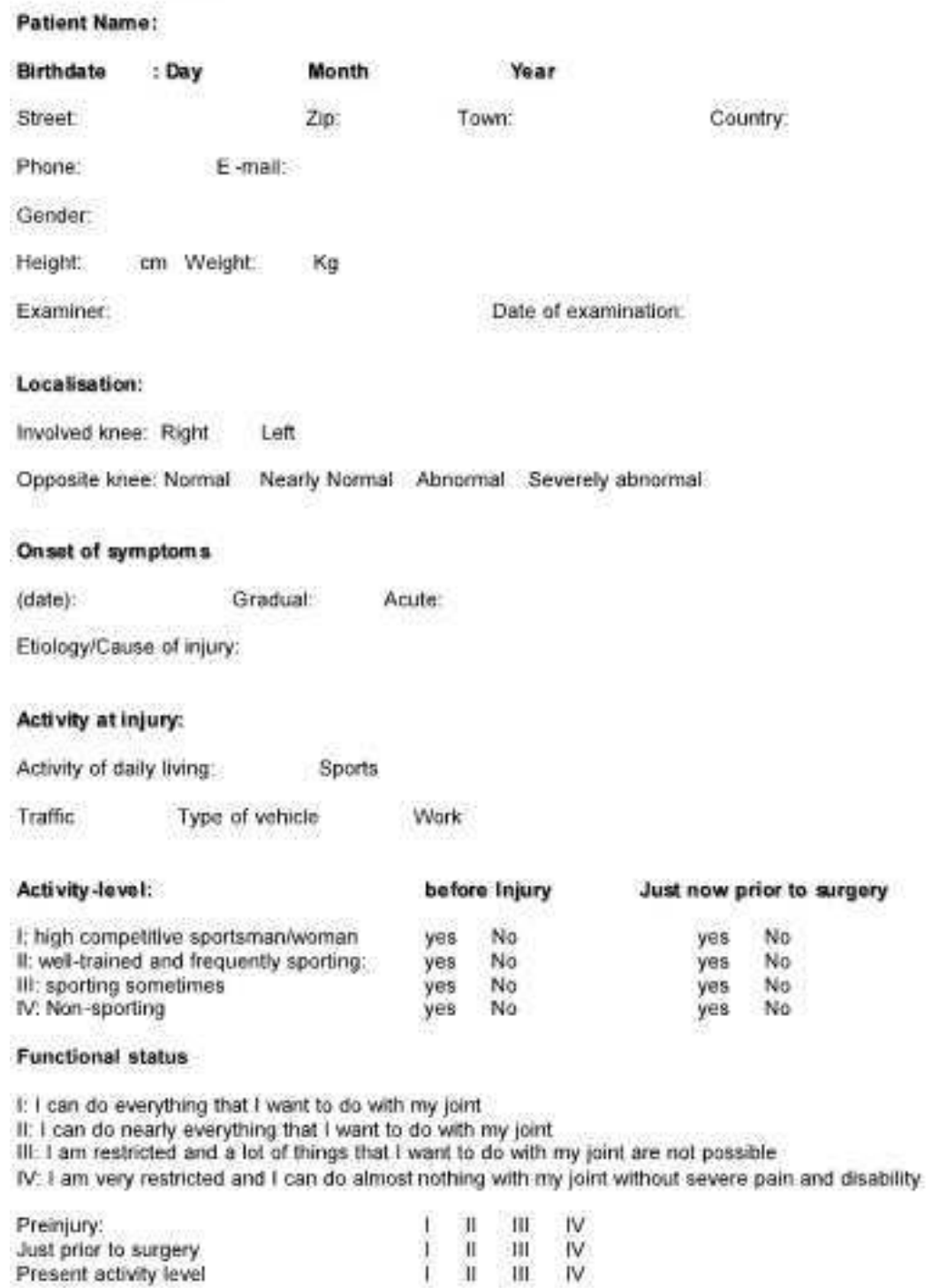

Abb. 38: $\quad$ ICRS-Beurteilungspaket, S. 1 (Mit freundlicher Genehmigung der ICRS) 


\section{IKDC CuRRENT HEALth Assessment Form *}

\section{Patients Part:}

Your Full Name

Your Date of Birth

Today's Date

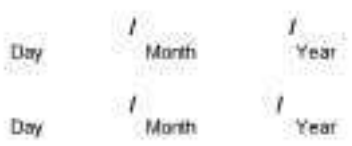

1. In general, would you say your health is:

Dexcelient

Dery Goot

DGood

a fair

DPaor

2 Compared to one year ago, how would you rate your health in general now?

DMuch betier now than 1 year ago

Dsomewhat better now than 1 year ago

Dhbout the same as 1 year ago

ISomewhat worse now than 1 yeat ago

Duch worse now than 1 year ago

3. The following items are about activities you might do during a typical day. Does your health now limit you in these activities? If 50 , how much?

$\begin{array}{ccc}\text { Yes: } \\ \text { Limited } \\ \text { ALOt } & \begin{array}{c}\text { Yos: } \\ \text { Limited } \\ \text { A Little }\end{array} & \begin{array}{c}\text { No, Not } \\ \text { Limited } \\ \text { Al All }\end{array} \\ \square & \square & \square \\ \square & \square & \square \\ \square & \square & \square \\ \square & \square & \square \\ \square & \square & \square \\ \square & \square & \square \\ \square & \square & \square \\ \square & \square & \square \\ \square & \square & \square \\ \square & \square & \square\end{array}$

Abb. 39: ICRS-Beurteilungspaket, S. 2

(Mit freundlicher Genehmigung der ICRS) 


\section{Anhang}

4. During the past 4 weaks, have you had any of the following problems with your work or other regular daily activities as a result of your physical health?

$\begin{array}{lcr}\text { a. Cut down on the amount of time you spent on work or other activities } & \text { YES } & \text { NO } \\ \text { b. Accomplished less than you would like } & \square & \square \\ \text { c. Were limited in the kind of work or other activities } & \square \\ \text { d. Had difficulty performing the work or other activities (for example, it took } & \square & \square\end{array}$

5. During the past 4 weeks have you had any of the following problems with your work or other regular daily activities as a result of any emotional problems (such as feeling depressed or anxious)?

\begin{tabular}{lcc} 
a. Cut down on the amount of time you spent on work or other activities & YES & NO \\
b. Accomplished less than you would like & $\square$ & $\square$ \\
c. Didn't do work or other activities as carefully as usual & $\square$ & $\square$ \\
\hline
\end{tabular}

6. During the past 4 weeks, to what extent has your physical health or emotional problems interfered with your normal social activities with family, friends, neighbors, or groups?

$\square$ Not At All

DSlightly

Doderately

DQuite a Bit

Extremely

7. How much bodily pain have you had during the past 4 weeks?

$\square$ None

Wery Mild

口Mild

Moderate

DSevere

Dery Severe

8. During the past 4 weeks, how much did pain interfere with your normal work fincluding both work outside the home and housework)?

DNot at All

aA Littie Bit

$\square$ Moderately

DQuite a Bit

DExtremely

Abb. 40: ICRS-Beurteilungspaket, S. 3 (Mit freundlicher Genehmigung der ICRS) 
9. These questions are about how you feel and how things have been with you during the past 4 weeks. For each question, please give the one answer that comes closest to the way you have been feeling. How much of the time during the past 4 weeks...

$\begin{array}{lllllll}\text { a. Did you feel full of pep? } & \square & \square & \square & \square & \square & \square \\ \text { b. Have you been wery nervous? } & \square & \square & \square & \square & \square & \square \\ \text { c. Have you fell calm and peaceful? } & \square & \square & \square & \square & \square & \square \\ \text { d. Did you have a lot of energy? } & \square & \square & \square & \square & \square & \square \\ \text { e. Have you felt down-hearted and blue? } & \square & \square & \square & \square & \square & \square \\ \text { f. Did you feel wom out? } & \square & \square & \square & \square & \square & \square \\ \text { g. Have you been a happy person } & \square & \square & \square & \square & \square & \square \\ \text { h. Did you feel tired? } & \square & \square & \square & \square & \square & \square\end{array}$

10. During the past 4 weeks how much of the time has your physical health or emotional problems interfered with your social activities (like visiting with friends, relatives, etc.)?

DAll of the time

$\square$ Most of the time

$\square$ Some of the time

A little of the time

$\square$ None of the time

11. How TRUE or FALSE is each of the following statements for you?

$\begin{array}{lccccc} & \text { Definitely } & \text { Mostly } & \text { Don' Mostly Definilely } \\ \text { a I seem to get sick a little easier than other people } & \text { True } & \text { True } & \text { Know False False } \\ \text { h. I am as healthy as anybody I know } & \square & \square & \square & \square \\ \text { c. I expect my health to get worse } & \square & \square & \square & \square \\ \text { d My health is excellent } & \square & \square & \square & \square & \square\end{array}$

"This form includes questions from the SF-36" Health Survey. Reproduced with the permission of the Medical Outcomes Trust, Copyright \& 1992.

Abb. 41: ICRS-Beurteilungspaket, S. 4 (Mit freundlicher Genehmigung der ICRS) 
2000 IKDC Subjective Knee Evaluation Form Patients Part:

Your Full Name

Today's Date: Day 'Month 'Year Date of injury Day 'Month 'Year

\section{SYMPTOMS:}

"Grade symptoms at the highest activity lewel at which you thirk you could function without significant symptoms. even if you are not actually performing activities at this level.

1. What is the highest level of activity that you can perform without significant knee pain?

QVery strenuous activities ike jumping or piwoting as in basketball or soccer

Dstrenuous activities like heawy physical work, sking or tennis

Woderate activities like moderate physical wock, running ar jogging

- Light activities like walking, housework or yard work

Uunable to pertorm any of the above activities due to knee pain

2 During the past 4 wenks, or since your injury, how often have you had pain?

$\begin{array}{lllllllllllll}\text { Never } & 0 & 1 & 2 & 3 & 4 & 5 & 6 & 7 & 8 & 9 & 10 & \\ \square & \square & \square & \square & \square & \square & G & 0 & a & \square & 0 & \text { Constant }\end{array}$

3. If you have pain, how severe is it?

\begin{tabular}{lllllllllllll} 
No pain & 0 & 1 & 2 & 3 & 4 & 5 & 6 & 7 & 8 & 9 & 10 & \\
\hline & $\square$ & $\square$ & $\square$ & $\square$ & $\square$ & $\square$ & $\square$ & $\square$ & $\square$ & $\square$ & Worst pain imaginable
\end{tabular}

4. During the past 4 weeks, or since your injury, how stiff or swollen was your knee?

UNot at all

QMildiy

Woderately

Quvery

Extremely

5. What is the highest level of activity you can perform without significant swelling in your knee?

$\square$ Very strenuous activities like jumping or pivoting as in baskethall or soccer

QStrenuous activities like heavy physical work, skäng or ternis

Woderate activities like moderate physical work, nunning or jogging

ULight activities like walking, housework, or yard work

Gunable to perform any of the above activities due to knee swelling

6. During the past 4 weeks, or since your injury, did your knee lock or catch?

Dres ఏNo

2. What is the highest level of activity you can perform without significant giving way in your knee?

Query strenuous activities like jumping or pivoting as in basketball or soccer

QStrenuous activities like heavy physical work, sking or tennis

QModerate activities like moderate physical work; running or jogging

D light activities like walking, housework or yard work

UUnable to perform any of the above activities due to giving way of the knee

Abb. 42: ICRS-Beurteilungspaket, S. 5

(Mit freundlicher Genehmigung der ICRS) 
SPORTS ACTIVITES:

a. What is the highest level of activity you can participate in on a regular basis?

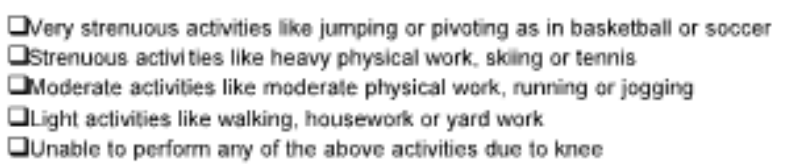

2. How does your knee affect your ability to:

\begin{tabular}{|l|l|c|c|c|c|c|}
\hline & & $\begin{array}{c}\text { Not difficult } \\
\text { at all }\end{array}$ & $\begin{array}{c}\text { Minimally } \\
\text { difficult }\end{array}$ & $\begin{array}{c}\text { Moderately } \\
\text { Difficult }\end{array}$ & $\begin{array}{c}\text { Extremely } \\
\text { difficult }\end{array}$ & $\begin{array}{c}\text { Unable } \\
\text { to do }\end{array}$ \\
\hline a & Go up stairs & $\square$ & $\square$ & $\square$ & $\square$ & $\square$ \\
\hline b. & Go down stairs & $\square$ & $\square$ & $\square$ & $\square$ & $\square$ \\
\hline c. & Kneel on the front of your knee & $\square$ & $\square$ & $\square$ & $\square$ & $\square$ \\
\hline d & Squat & $\square$ & $\square$ & $\square$ & $\square$ & $\square$ \\
\hline e & Sit with your knee bent & $\square$ & $\square$ & $\square$ & $\square$ & $\square$ \\
\hline $\mathrm{f}$ & Rise from a chair & $\square$ & $\square$ & $\square$ & $\square$ & $\square$ \\
\hline g & Run straight ahead & $\square$ & $\square$ & $\square$ & $\square$ & $\square$ \\
\hline h & Jump and land on your imvolved leg & $\square$ & $\square$ & $\square$ & $\square$ & $\square$ \\
\hline $\mathrm{i}$. & Stop and start quickly & $\square$ & $\square$ & $\square$ & $\square$ & $\square$ \\
\hline
\end{tabular}

\section{FUNCTION:}

10. How would you rate the function of your knee on a scale of 0 to 10 with 10 being normal, excellent function and 0 being the inability to perform any of your usual daily activities which may include sports?

FUNCTION PRIOR TO YOUR KNEE INJURY:

$$
\begin{aligned}
& \text { Cannot perform } \\
& \text { daily activities } \\
& \begin{array}{lllllllllll}
0 & 1 & 2 & 3 & 4 & 5 & 6 & 7 & 8 & 9 & 10 \\
\square & \square & \square & \square & \square & \square & \square & \square & \square & \square & \square
\end{array}
\end{aligned}
$$

CURRENT FUNCTION OF YOUR KNEE:

$$
\begin{aligned}
& \begin{array}{l}
\text { Cannot perform } \\
\text { daily activities }
\end{array} \\
& \\
& 0
\end{aligned}
$$

Abb. 43: ICRS-Beurteilungspaket, S. 6 (Mit freundlicher Genehmigung der ICRS) 


\section{Anhang}

\section{SCORING INSTRUCTIONS FOR THE 2000 IKDC SUBJECTIVE KNEE EVALUATION FoRM}

Several methods of scoring the IKDC Subjective Knee Evaluation Form were investigated. The results indicated that summing the scores for each item performed as well as more sophisticated scoring methods.

The responses to each item are scored using an ordinal method such that a score of 1 is given to responses that represent the lowest level of function or highest level of symptoms. For example, item 1, which is related to the highest level of activity without significant pain is scored by assigning a score of 1 to the response "Unable to Perform Any of the Above Activities Due to Knee" and a score of 5 to the response "Very strenuous activities like jumping or pivoting as in basketball or socoer". For item 2, which is related to the frequency of pain over the past 4 weeks, the response "Constant" is assigned a score of 1 and "Never' is assigned a score of 11.

The IKDC Subjective Knee Evaluation Form is scored by summing the scores for the individual items and then transforming the score to a scale that ranges from 0 to 100 . Note: The response to item 10 'Function Prior to Knee Injury" is not included in the overall soore. The steps to score the IKDC Subjective Knee Evaluation Form are as follows:

1. Assign a score to the individual's response for each item, such that lowest score represents the lowest level of function or highest level of symptoms.

2. Calculate the raw score by summing the responses to all items with the exception of the response to item 10 "Function Prior to Your Knece Iniury"

3. Transform the raw score to a 0 to 100 scale as follows:

$$
\text { IKDC Score }=\left[\frac{\text { Raw Score }- \text { Lowest Possible Score }}{\text { Range of Scores }}\right] \times 100
$$

Where the lowest possible score is 18 and the range of possible scores is 87 . Thus, if the sum of scores for the 18 intems is 60 , the IKDC Score would be calculated as follows:

$$
\text { IKDC Score }=\left[\frac{60-18}{87}\right] \times 100
$$

IKDC Score $=48.3$

The transformed score is interpreted as a measure of function such that higher scores represent higher levels of function and lower levels of symptoms. A score of 100 is interpreted to mean no limitation with activities of dally living or sports activities and the absence of symptoms.

The IKDC Subjective Knee Score can still be calculated if there are missing data, as long as there are responses to at least $90 \%$ of the inems (i.e. responses have been provided for at least 16 items). To calculate the raw IKDC score when there are missing data, substitute he average score of the items that have been answered for the missing item score(s). Once the raw IKDC score has been calculated, it is transformed to the IKDC Subjective missing item score(s). Once the
Knee Score as described above.

Abb. 44: ICRS-Beurteilungspaket, S. 7 (Mit freundlicher Genehmigung der ICRS) 
ICRS KNeE HISTORY REgistration-PREVIOUS SURgery Surgeons part

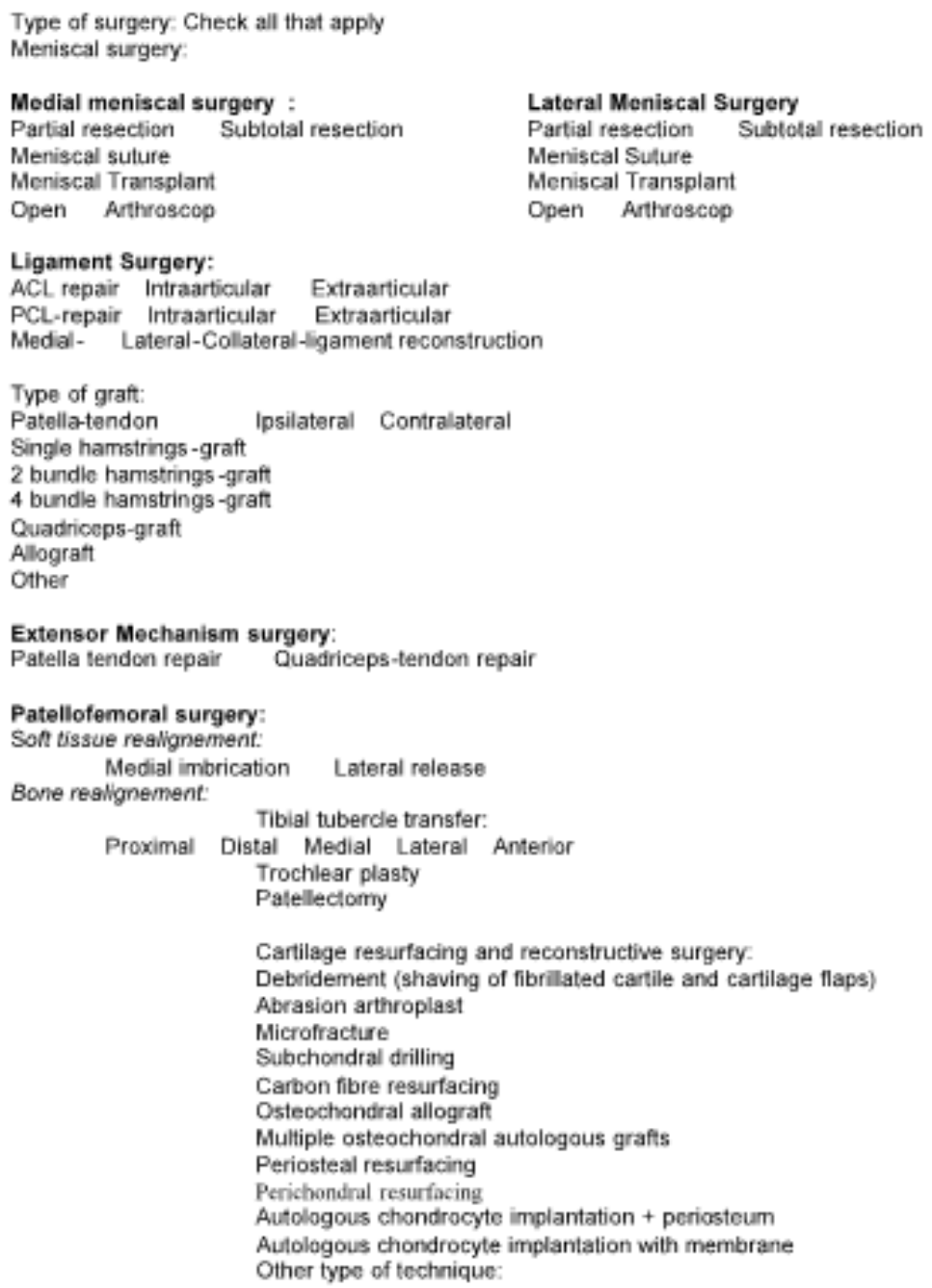

Abb. 45: ICRS-Beurteilungspaket, S. 8

(Mit freundlicher Genehmigung der ICRS) 
Surgeons part

\begin{tabular}{|c|c|c|c|c|c|}
\hline & Osteotomy: & Tibia & Femur & Varus & Valgus \\
\hline & Plain x-rays: & & Varus : & & Valqus-angle \\
\hline
\end{tabular}

Findings:

Articular cartilage appearance:

Bone:

Ligaments:

Menisci:

Abb. 46: ICRS-Beurteilungspaket, S. 9

(Mit freundlicher Genehmigung der ICRS) 
2000 IKDC KNEE Examination Form

Surgeons part

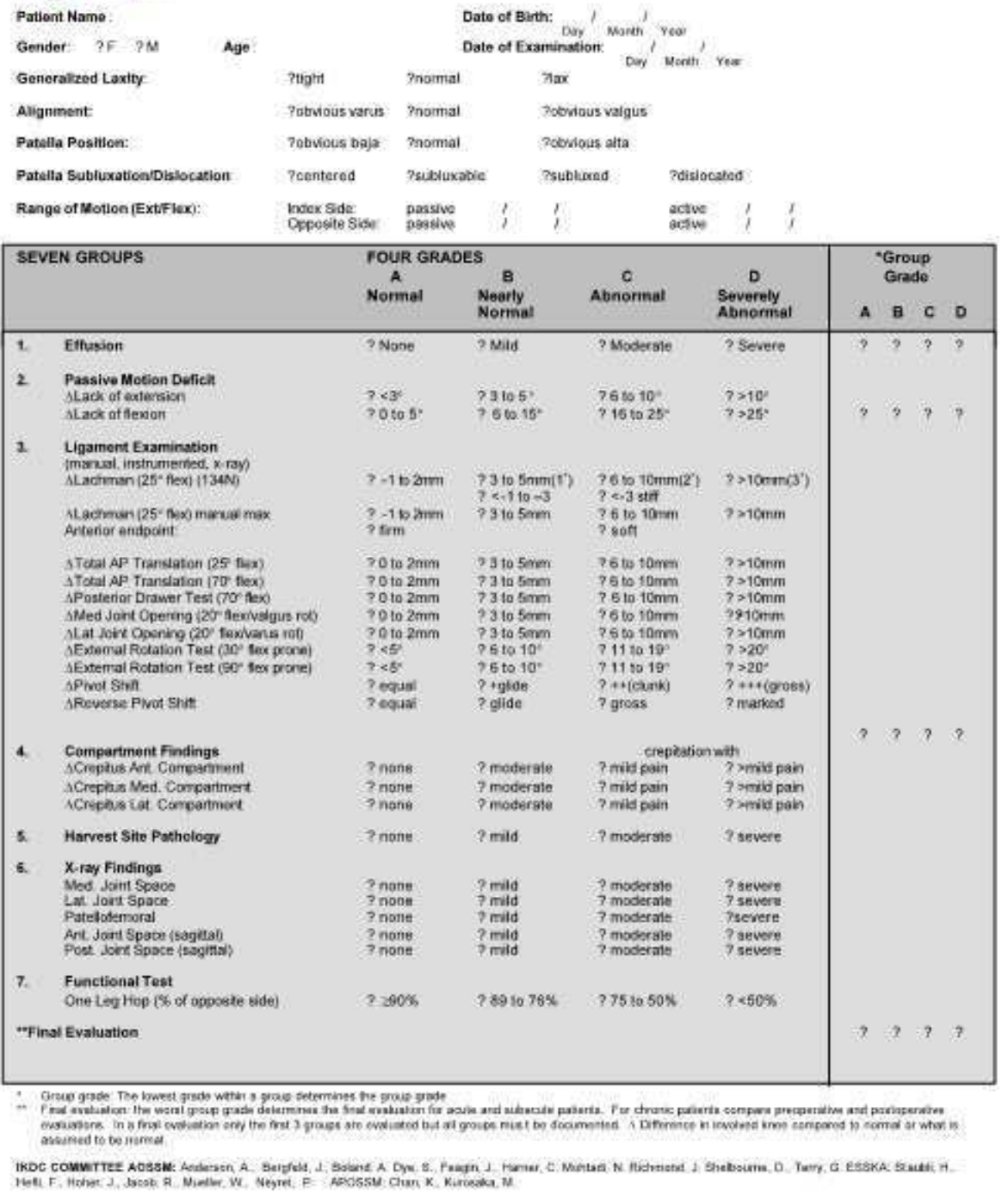

Abb. 47: ICRS-Beurteilungspaket, S. 10 (Mit freundlicher Genehmigung der ICRS) 


\section{Anhang}

\section{INSTRUCTIONS FOR THE 2000 IKDC KNEE EXAMINATION FORM}

The Knee Examination Form contains items that fall into one of seven measurement domains. However, only the first three of these domains are graded. The seven domains assessed by the Knee Examination Form are:

1. Ethrsion

An effusion is assessed by ballotting the knee. A fluid wave (less than 25 cc) is graded mild, easily ballotteable fuid - moderale $(25-60 \mathrm{cc})$, and a tense knee secondary to effusion (greater than $60 \mathrm{cc}$ ) is fated severe.

2. Passive Motion Deficit

Passive range of motion is measured with a gonimeter and recorded on the form for the index side and opposite of normal side. Record values for zero poinWhyperextensionmexion (e.g. 10 degrees of hyperextension, 150 degrees of flexion $=100 / 150$; 10 degrees of fexion to 150 degrees of flexion $=$ 0i10 150). Extension is compared to that of the normal knee,

3. Ligament Examination

The Lachman test, total AP translation at 70 degrees, and medial and lateral joint opening may be assessed with manual, instrumented or stress, wray examination. Only one should be graded, preferably a "measured displacement". A force of $134 \mathrm{~N}$ (30 lbs) and the maximum manual are recorded in instrumented examination of both knees. Only the measured displacement at the standard force of $134 \mathrm{~N}$ is used for grading. The numerical values for the side to side difference are rounded off, and the appropriate box is marked.

The end point is assessed in the Lachman test. The end point affects the grading when the index knee has 3-5 mm more anterior laxity than the nomal knee. In this case, a sof end poirt results in an abnormal grade rather than a nearly normal grade.

The 70-degree posterior sag is estimated by comparing the profle of the injured knee to the normal knee and palpating the medial femoral tibla step off. it may be confirmed by noting that contraction of the quadriceps pulls the tibia interiorly.

The external rotation tests are performed with the patient prone and the knee flexed $30^{*}$ and 70 .

Equal extemal rotational torque is applied to both feet and the degree of external rotation is recorded.

The pivot shift and rewerse pivot shit are performed with the patient supine, with the hip in $10-20$ degrees of abduction and the obia in neutral rotation using either the Losee, Noyes, or Jakob techniques. The greatest subluxation, compared to the normal knee, should be recorded.

4. Compartment Findings

Patellofemoral crepitation is elicited by extension against slight resistance. Medial and lateral compartment crepitation is elicited by extending the knee from a fexed position with a varus stress and then a valgus stress (i.e., McMurray test). Grading is based on intensity and pain.

5. Hanvest Sile Pachalogy Note tenderness, irritation or numbness at the autograft harvest site.

6. X-ray Findings

A bilateral, double leg PA weightbearing roentgenogram at 35-45 degrees of flexion (tunnel view) is used to evaluate narrowing of the medial and lateral joint spaces. The Merchant view at 45 degrees is used to document patellafemoral narrowing. A mild grade indicates minimal changes (i.e., small osteophytes, slight sclerosis or flattening of the femoral condyle) and narrowing of the joint space which is just detectable. A moderate grade may have thase chenges and joint space narrowing (e g. a joint space of 2-4 mm side or up to $50 \%$ joint space narrowing). Severe changes include a joint space of less than $2 \mathrm{~mm}$ or greater than $50 \%$ joint space narrowing.

7. Functional Test

The patient is asked to perform a one leg hop for distance on the index and normal side. Three trials for each leg are recorded and averaged. A ratio of the index to normal knee is calculated.

Abb. 48: ICRS-Beurteilungspaket, S. 11 (Mit freundlicher Genehmigung der ICRS) 
ICRS Grade 0 - Normal

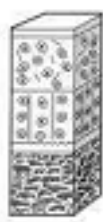

ICRS Grade 1 - Nearly Normal

Superficial lesions. Soft indertation (A) andjor superficial fissures and cracks (B)
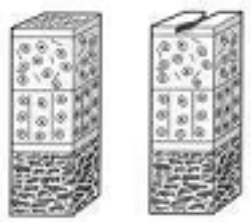

ICRS Grade 2 - Abnormal

Lesions extending down to $<50 \%$ of cartiage depth

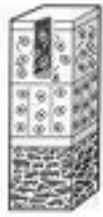

ICRS Grade 3 - Severely Abnormal

Cartilage defects extending down $>50 \%$ of cartilage depth (A) as wel as down to calcified layer (B) and down to but not through the subchondral bone (C). Blsters are included in this Grade (D)

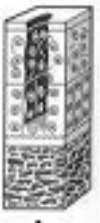

A

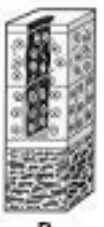

B

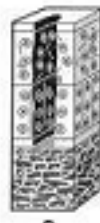

c

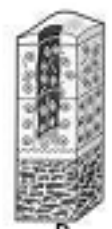

D

ICRS Grade 4- Severely Abnormal
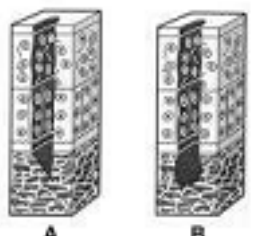

Copyright $\odot$ ICRS

Abb. 49: ICRS-Beurteilungspaket, S. 12

(Mit freundlicher Genehmigung der ICRS) 


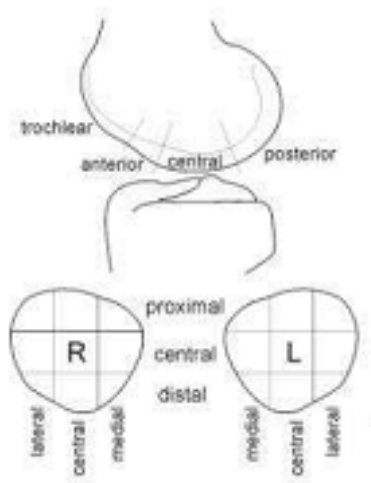

Measurement of the defect size: $\square 11 \mathrm{~mm}$
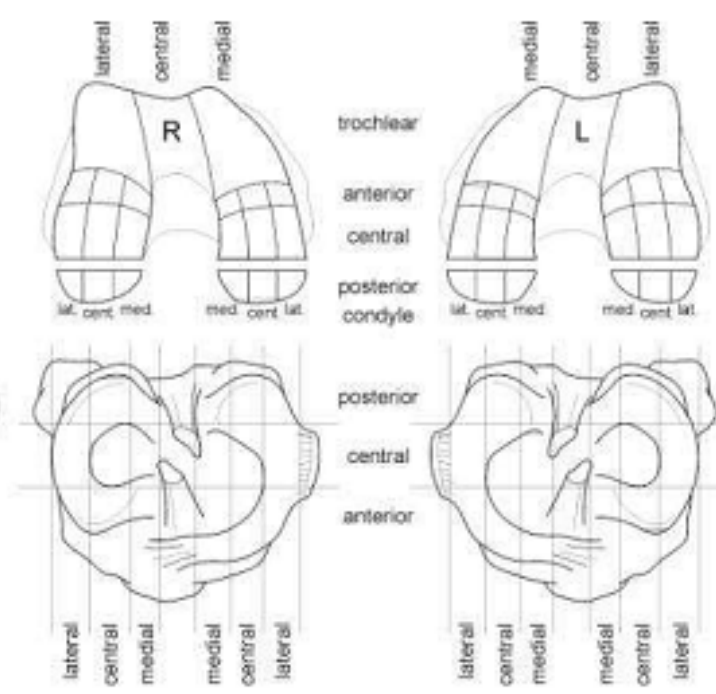

posterior

central

anterior

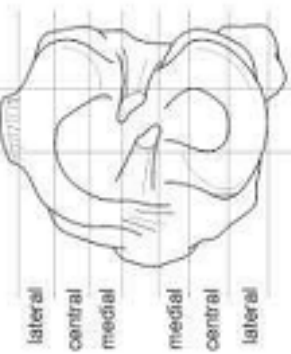

Femur

Side

Right Left

Condyle medial lateral

Sagital plane trochlear anterior entral posterior

Frontal plane lateral central medial

First lesion

Second lesion

Cartiage lesion (Grade) (')

Defect size post-debridement

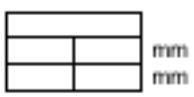

Tibia

Side Right Left

Plateau medal lateral

Sagital plane anterior central posterior

Frontal plane lateral central medial

Cartilage iesion (Grade) (")

Defect size pre-debridement

Defect size post-debridement

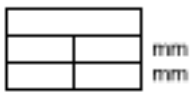

Patella
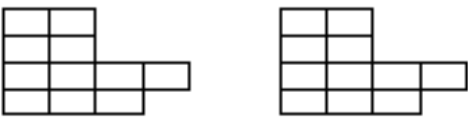

Side

Right Left

Sagital plane distal central proximal

Frontal plane lateral central medial

Cartiage lesion (Grade) (")

Defect size pre-debridement

Defect size post-debridement

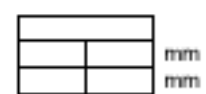

Diagnosis: $\quad \Pi$ Traumatic cartilage lesion $\quad \Pi \mathrm{OD}$

Biopsy/Osteochondral Plugs: Location:

IOA $\Pi$ AMN $I$ Others

Number of Plugs: Diameter of Plugs:

Treatment: П Shaving

r. Drilling

Mosaic-Plasty $\sqcup$ Microfracture

Autclogous Chondrocyle Implantation (ACI)

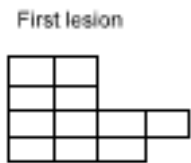

Second lesion

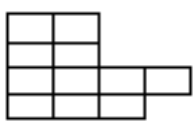

First lesion

Second lesion
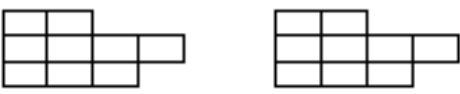

Others:

Notes:

Abb. 50: ICRS-Beurteilungspaket, S. 13

(Mit freundlicher Genehmigung der ICRS) 
ICRS Classification of OCD-Lesions (Osteochondritis-Dissecans)

ICRS OCD I

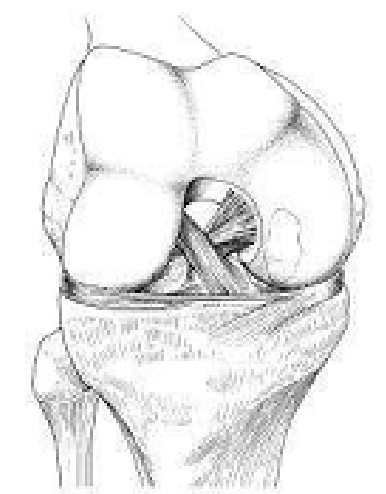

Stable, continuity: Saftened area covered by intact cartilage.
ICRS OCD ॥

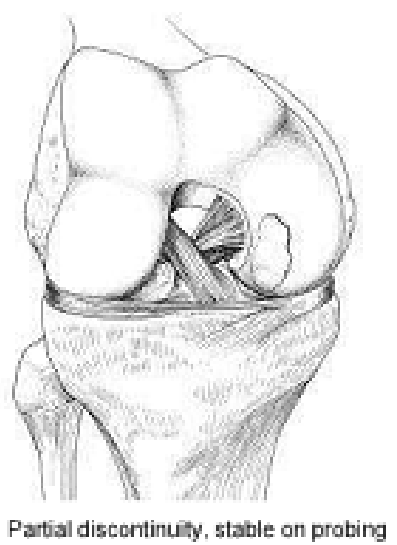

ICRS OCD IV

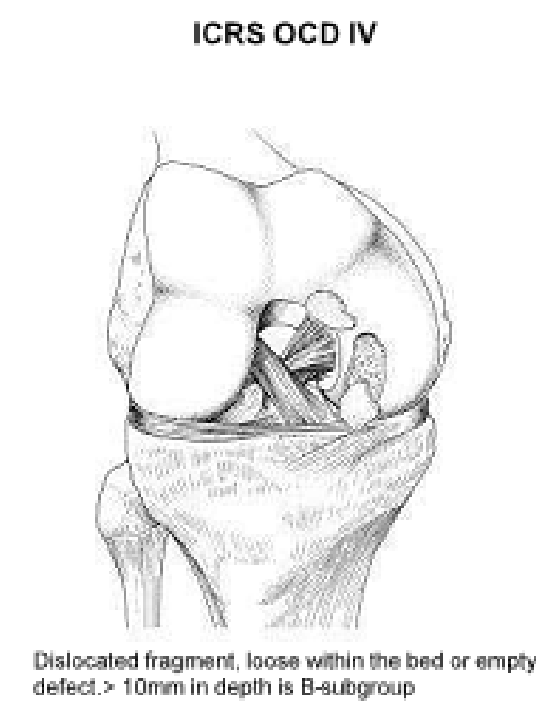

Dislocated fragment, loose within the bed or empty defect. $>10 \mathrm{~mm}$ in depth is B-subgroup

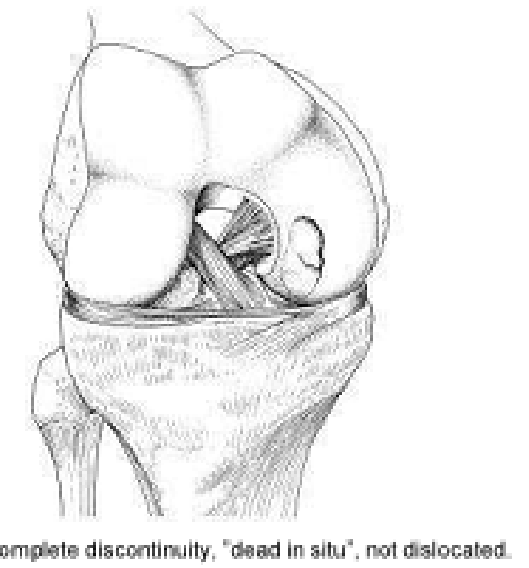

ICRS OCD III

Copyrigh o icrs

Abb. 51: ICRS-Beurteilungspaket, S. 14

(Mit freundlicher Genehmigung der ICRS) 


\section{Anhang}

Cartilage Repair Assessment

\begin{tabular}{|c|c|c|c|}
\hline Criteria & \multicolumn{2}{|l|}{ Points } & \\
\hline $\begin{array}{l}\text { Degree of Defect Repair } \\
\text { I Protocol A }\end{array}$ & \multicolumn{2}{|c|}{$\begin{array}{l}* \text { In level with surrounding cartilage } \\
-75 \% \text { repair of defect depth } \\
-50 \% \text { repair of defect depth } \\
-25 \% \text { repair of defect depth } \\
0 \% \text { repair of defect depth } \\
\end{array}$} & $\begin{array}{l}4 \\
3 \\
2 \\
1 \\
0\end{array}$ \\
\hline | Protocol B & \multicolumn{2}{|c|}{$\begin{array}{c}-100 \% \text { survival of initially grafted surface } \\
-75 \% \text { survival of initially grafted surface } \\
-50 \% \text { survival of initially grafted surface } \\
25 \% \text { survival of initially grafted surface } \\
0 \% \text { (plugs are lost or broken) }\end{array}$} & $\begin{array}{l}4 \\
3 \\
2 \\
1 \\
0 \\
\end{array}$ \\
\hline II Integration to Border zone & \multicolumn{2}{|c|}{$\begin{array}{l}\text { - Complete integration with surfounding cartilage } \\
\text { - Demarcating border }<1 \mathrm{~mm} \\
\text { - } 3 / 4 \text { of graft integrated, } 1 / 4 \text { with a notable border } \\
>1 \mathrm{~mm} \text { width } \\
-1 / 2 \text { of graft integrated with surrounding cartilage, } \\
1 / 2 \text { with a notable border }>1 \mathrm{~mm} \\
\text { - From no contact to } 1 / 4 \text { of graft integrated with } \\
\text { surrounding cartilage }\end{array}$} & $\begin{array}{l}4 \\
3 \\
2 \\
1 \\
1 \\
0\end{array}$ \\
\hline III Macroscopic Appearance & \multicolumn{2}{|c|}{$\begin{array}{l}\text { - Intact smooth surface } \\
\text { - Fibrillated surface } \\
\text { - Small, scattered fissures or cracs } \\
\text { - Several, small or few but large fissures } \\
\text { - Total degeneration of grafted area }\end{array}$} & $\begin{array}{l}4 \\
3 \\
2 \\
1 \\
0 \\
\end{array}$ \\
\hline Overall Repair Assessment & $\begin{array}{l}\text { Grade I } \\
\text { Grade II } \\
\text { Grade III } \\
\text { Grade IV }\end{array}$ & $\begin{array}{l}\text { normal } \\
\text { nearly normal } \\
\text { abnormal } \\
\text { severely abnormal }\end{array}$ & $\begin{array}{ll}12 & P \\
11-8 & P \\
7-4 & P \\
3-1 & P\end{array}$ \\
\hline
\end{tabular}

Cartilage Biopsy |+ Location

\begin{tabular}{|l|l|}
\hline $\begin{array}{l}\text { (1) Protocol A: } \\
\begin{array}{l}\text { autologous chondrocyte implantation (ACI); } \\
\text { periosteal or perichondrial transplantation; } \\
\text { subchondral drilling; microfracturing; carbon } \\
\text { fibre implants; others: }\end{array}\end{array}$ & $\begin{array}{l}\text { Mossaicplasty; OAT; osteochondral } \\
\text { allografts; others: }\end{array}$ \\
& \\
& \\
\hline
\end{tabular}

Abb. 52: ICRS-Beurteilungspaket, S. 15

(Mit freundlicher Genehmigung der ICRS) 


\section{LITERATURVERZEICHNIS}

Ahmad CS, Cohen ZA, Levine WN, Athesian GA, Mow VC (2001):

Biomechanical and Topographic Considerations for Autologous Osteochondral Grafting in the Knee

Am J Sports Med 29 , 201-206

Albrecht D, Weise K (2008):

[Therapy of articular cartilage lesions]

Chirurg $\underline{79}$, 989-998

Alford JW, Cole BJ (2005):

Cartilage restoration, part 1: basic science, historical perspective, patient evaluation, and treatment options.

Am J Sports Med $\underline{\mathbf{3 3}}$, 295-306

Årøen $\mathrm{A}$, Løken $\mathrm{S}$, Heir $\mathrm{S}$, Alvik $\mathrm{E}$, Ekeland $\mathrm{A}$, Granlund $\mathrm{OG}$, Engebretsen L (2004):

Articular cartilage lesions in 993 consecutive knee arthroscopies.

Am J Sports Med 르, 211-215

Aston JE, Bentley G (1986):

Repair of articular surfaces by allografts of articular cartilage and growth-plate cartilage.

J Bone Joint Surg Br $\underline{68}, 29-35$

Bauer M, Jackson RW (1988):

Condral Lesions of the Femoral Condyles : A System of Arthroscopic Classification.

Arthroscopy 4, 97-102

Bethge JF (1962):

Chondromalazia patellae.

Dtsch Med Wochenschr 87, 636-643

Bhakta NR, Garcia AM, Frank EH, Grodzinsky AJ, Morales TI (2000):

The insulin-like growth factors (IGFs)I and II bind to articular cartilage via the IGFbinding proteins.

J Biol Chem 257, 5860-5866

Bläsius K, Hoeckle C, Karkour I, Guinard M:

Nachbehandlungsfibel Orthopädie und Unfallchirurgie.

Thieme-Verlag, Stuttgart, 2008, 110

Bobic V (1996):

Arthroscopic osteochondral autograft transplantation in anterior cruciate ligament reconstruction : a preliminary clinical study.

Knee Surg Sports Traumatol Arthrosc יㅜ, 262-264 
Bös L, Ellermann A (2003):

Arthroskopische Diagnostik und Klassifikation von Knorpelschäden.

Dtsch Z Sportmed $\underline{\mathbf{5 4}}, 181-183$

Böttner :

Facharztkompendium Orthopädie und Unfallchirurgie.

OrthoForum-Verlag, Berlin, 2008, 198

Braun S, Vogt S, Imhoff AB (2007):

Stadiengerechte operative Knorpeltherapie.

Orthopade 36, 589-600

Brittberg M, Winalski CS (2003):

Evaluation of cartilage injuries and repair.

J Bone Joint Surg Am 85, 58-69

Brittberg M, Lindahl A, Nilsson A, Ohlsson C, Isaksson O, Peterson L (1994):

Treatment of deep cartilage defects in the knee with autologous chondrocyte transplantation.

N Engl J Med 331, 889-895

\section{Bruns J:}

Osteochondrosis dissecans. Pathogenese, Diagnose und Therapie.

Enke-Verlag, Stuttgart, 1996

\section{Buckwalter JA (1995):}

Osteoarthritis and articular cartilage use, disuse and abuse: Experimental studies.

J Rheumatol 푸, 13-15

Buckwalter JA, Lohmander S (1994):

Current concepts review: operative treatment of osteoarthrosis.

J Bone Joint Surg Am 트, 1405-1408

Buckwalter JA, Mankin HJ (1998):

Articular Cartilage: Degeneration and osteoarthritis, repair, regeneration, and transplantation.

Instr Course Lect 47, 487-504

Buckwalter JA, Mow VC, Ratcliffe A (1994):

Restoration of Injured or Degenerated Articular Cartilage.

Am J Acad Orthop Surg 2 , 192-201

Bugbee WD, Convery FR (1999):

Osteochondral allograft transplantation.

Clin Sports Med 18, 67-75

Bullinger M, Kirchberger I:

SF-36 Fragebogen zum Gesundheitszustand.

Hogrefe-Verlag, Göttingen, 1998 
Chow JCY, Hantes ME, Houle JB, Zalavras CG (2004):

Arthroscopic Autogenous Osteochondral Transplantation for Treating Knee Cartilage Defects : A 2- to 5-Year Follow-up Study.

Arthroscopy 20, 681-690

Coen MJ, Caborn DN, Johnson DL (1996):

The dipling phenomenon: articular cartilage injury overlying an occult osteochondral lesion at the time of anterior cruciate ligament reconstruction-

Arthroscopy 12, 502-505

Cole BJ, Frederick RW, Levy AA, Zaslav KR (1999):

Management of a 37 year old man with recurrent knee pain.

$J$ Clin Outcomes Manag $\underline{6}, 46-57$

Curl WW, Krome J, Gordon ES, Rushing J, Smith BP, Poehling GG (1997):

Cartilage injuries: A review of 31,516 knee arthroscopies.

Arthroscopy 13, 456-460

Dew TL, Martin RA (1992):

Functional, radiographic, and histologic assessment of healing of autogenous osteochondral grafts and full-thickness cartilage defects in the talus of dogs.

Am J Vet Res $\underline{\mathbf{5 3}}, 2141-2152$

Engebretsen L, Bahr R (2004):

Surgical treatment of cartilage injuries

Eur Surg 으, 13-19

Ficat RP, Hungerford DS:

Disorders of the patellofemoral joint.

Masson, Paris, 1977, 123

Figueroa D, Calvo R, Vaisman A, Carrasco MA, Moraga C, Delgado I (2007):

Knee Chondral Lesions: Incidence and Correlation Between Arthroscopic and Magnetic Resonance Findings.

Arthroscopy 23, 312-315

\section{Freemont AF:}

Pathophysiology of Osteoarthritis.

In: Arden N, Cooper C (eds) osteoarthritis Handbook.

Taylor and Francis, London, 2006, 89

Fritz J, Aicher WK, Eichhorn HJ:

Praxisleitfaden der Knorpelreparatur.

Springer-Verlag, Berlin, 2003

Geneser F:

Histologie.

Deutscher Ärzte-Verlag, Köln, 1990 
Gray ML, Pizzanelli AM, Grodzinsky AJ, Lee RC (1988):

Mechanical and physicochemical determinants of the chondrocyte biosynthetic response.

J Orthop Res $\underline{6}, 777-792$

Hangody L, Füles P (2003):

Autologous osteochondral mosaicplasty for the treatment of full-thickness defects of weight-bearing joints. Ten years of experimental and clinical experience.

J Bone Joint Surg Am 모, 25-32

Hangody L, Ráthonyi GK, Duaska Z, Vásárhelyi G, Füles P, Módis L (2004):

Autologous osteochondral mosaicplasty. Surgical technique.

J Bone Joint Surg Am $\underline{\mathbf{8 6}}, 65-72$

Hayes DW, Averett RK (2001):

Articular cartilage transplantation. Current and future limitations and solutions.

Clin Podiatr Med Sur 18, 161-176

Hempfling $\mathrm{H}$, Weise K (2007):

Begutachtung des Knorpelschadens

DGU - Mitt Nachr ${ }^{1} \underline{\text { 29}}$, 3-60

Hille E (1988) :

Arthroskopische Behandlung des Patella-Femoral Gelenkes.

Arthroskopie 1, 124-131

Hjelle K, Solheim E, Strand T, Muri R, Brittberg M (2002):

Articular cartilage defects in 1,000 knee arthroscopies.

Arthroscopy 18, 730-734

Hunter W (1995):

Of the structure and diseases of articulating cartilages. 1743.

Clin Orthop Relat Res 317, 3-6

Huntley JS, McBirnie JM, Simpson AH, Hall AC (2005):

Cutting-edge design to improve cell viability in osteochondral grafts.

Osteoarthrits Cartilage $\underline{\mathbf{1 3}}, 665-671$

Imhoff AB, Baumgartner R, Linke RD:

Checkliste Orthopädie.

Thieme-Verlag, Stuttgart, 2006

Jerosch J, Floren M (2000):

Lebensqualitätsgewinn (SF-36) nach Implantation einer Knieendoprothese.

Unfallchirurg 103, 371-374

Johnson LL (1986):

Arthroscopic abrasion arthroplasty historical and pathologic perspective: present status.

Arthroscopy 2, 54-69 
Junqueira LC, Carneiro J, Kelley RO:

Histologie.

Springer-Verlag, Berlin, 2002

Kish G, Modis L, Hangody L (1999):

Osteochondral mosaicplasty for the treatment of focal chondral and osteochondral lesions of the knee and talus in the athlete.

Clin Sports Med 18, 45-66

Klinger HM, Baums MH, Otte S, Steckel H (2003):

Anterior cruciate reconstruction combined with autologous osteochondral transplantation.

Knee Surg Sports Traumatol Arthrosc 11, 366-371

\section{Kohn D:}

Das Knie.

Thieme-Verlag, Stuttgart, 2000

Koulalis D, Schultz W, Heyden M, König F (2004):

Autologous osteochondral gafts in the treatment of cartilage defects of the knee joint. Knee Surg Sports Traumatol Arthrosc 12, 329-334

\section{Lahm A:}

Sekundärschaden des Knorpels nach subchondralen Verletzungen des Kniegelenkes. Histologische und immunhistochemische Untersuchungen nach isolierten kernspintomographisch nachgewiesenen knöchernen Läsionen.

Med. Habil. Freiburg i. Br., 2002

\section{Laprell H, Petersen W (2001):}

Autologous osteochondral transplantation using the diamond bone-cutting system (DBCS): 6-12 years'follow-up of 35 patients with osteochondral defects at the knee joint.

Arch Orthop Trauma Surg 121, 248-253

\section{Löffler G, Petrides PE:}

Biochemie und Pathobiochemie.

Springer-Verlag, Berlin, 2003

\section{Lyons TJ, Stoddart RW, McClure SF, McClure J (2005):}

The tidemark of the chondro-osseus junction of the normal human knee joint. J Mol Histol 36, 207-215

Mallinger R, Geleff S, Böck P (1986):

Histochemistry of glycosaminoglycans in cartilage ground substance. Alcian-blue staining and lectin-binding affinities in semithin Epon sections.

Histochemistry $\underline{\mathbf{8 5}}, 121-127$

Marcacci M, Kon E, Delcogliano M, Filarda G, Busacca M, Zaffignini S (2007):

Arthroscopic autologous osteochondral grafting for cartilage defects of the knee: prospective study results at a minimum 7-year follow-up.

Am J Sports Med 12, 2014-2021 
Martinek V (2003):

Anatomie und Pathophysiologie des hyalinen Knorpels.

Dtsch Z Sportmed $\underline{\mathbf{5 4}}, 166-170$

Meenen NM, Rischke B (2003):

Autogene osteochondrale Transplantation (AOT) bei Knorpeldefekten am Femurkondylus

Oper Orthop Traumatol 15, 38-56

Metcalf RW (1982):

An arthroscopic ,ethod for lateral release of the subluxating or dislocating patella.

Clin Orthop Relat Res 167, 9-18

Mow VC, Holmes MH, Plander KD (1984):

Fluid transport and mechanical properties of articular cartilage: a review.

J Biomech 17, 377-394

Outerbridge RE (1961):

The etiology of chondromalacia patellae.

$J$ Bone Joint Surg Br $\underline{43}, 752-757$

Palmoski MJ, Brandt KD (1984):

Effects of static and cyclic compressive loading on articular cartilage plugs in vitro. Arthritis Rheum 27, 675-681

Petersen W, Zantop T, Tillmann B (2006):

Anatomie des hinteren Kreuzbandes sowie der posterolateralen und posteromedialen Strukturen.

Arthroskopie 19 $198-206$

Potter HG, Linklater HM JM, Allen AA et al. (1998):

Magnetic resonance imaging of articular cartilage in the knee. An evaluation with use of fast-spin-echo imaging.

J Bone Joint Surg Am 80, 1276-1284

Pridie KH (1959):

A method of resurfacing osteoarthic knee joints.

$J$ Bone Joint Surg Br 뚜, 618-619

Puhl W (1997):

[Arthrosis]

Z Orthop Ihre Grenzgeb 135, 183

Rauber A, Kopsch F:

Anatomie des Menschen, Band 1 Bewegungsapparat.

Thieme-Verlag, Stuttgart, 1987

Rolauffs B, Aicher WK, Ochs BG, Bahrs C, Albrecht D, Weise K (2009):

Regenerative Medizin zur Behandlung von Knorpelschäden

Orthop Unfallchir up2date ${ }^{1} \mathbf{4}, 339-354$ 
Rudert M, Wirth CJ (1998):

Knorpelregeneration und Knorpelersatz.

Orthopade 27, 309-321

Sah RL, Kim YJ, Doong JY, Grodzinsky AJ, Plaas AH, Sandy JD (1998):

Biosynthetic response of cartilage explants to dynamic compression.

J Orthop Res $\underline{7}, 619-636$

Schiebler TH, Schmidt W, Zilles K:

Anatomie.

Springer-Verlag, Berlin, 1995

Schlitt HJ:

Lebensqualität nach Transplantation.

Thieme-Verlag, Stuttgart, 2006

Sellards RA, Nho SJ, Cole BJ (2002):

Chondral injuries.

Curr Opin Rheumatol 14, 134-141

Sharpe JR, Ahmed SU, Fleetcroft JP, Martin R (2005):

The treatment of osteochondral lesions using a combination of autologous chondrocyte implantation and autograft: three-year follow-up.

J Bone Joint Surg Br 87, 730-735

Shasha N, Krywulak S, Backstein D, Pressman A, Gross AE (2003):

Long-term follow-up of fresh tinial osteochondral allografts for for failed tibial plateau fracture.

J Bone Joint Surg Am 85, 33-39

Smith GD Smith GD, Taylor J, Almqvist KF, Erggelet C, Knutsen G, Garcia Portabella M, Smith T, Richardson JB (2005):

Arthroscopic assessment of cartilage repair: A validation study of two scoring systems.

Arthroscopy 21, 1462-1467

Spahn G, Wittig R (2003):

Spannungs- und Bruchverhalten des gesunden Gelenkknorpels unter axialer Belastung. Eine biomechanische Untersuchung

Zentralb/ Chir 128, 78-82

Spahn G, Wittig R, Kahl E, Klinger HM, Mückley T, Hoffmann GO (2007):

Diagnostik von Knorpelschäden des Kniegelenks Validität der klinischen, radiologischen und kernspintomographischen Diagnostik im Vergleich zur Arthroskopie.

Unfallchirurg 110, 414-424

Steadman (2001):

Microfracture technique for full-thickness chondral defects: Technique and clinical results.

Oper Tech Orthop $\underline{7}, 300-304$ 
Steadman JR, Rodkey WG, Singleton SB, Briggs KK (1997):

Microfracture technique for full-thickness chondral defects: Technique and clinical results.

Oper Tech Orthop $\underline{7}, 300-304$

Szerb I, Hangody L, Duska Z, Kaposi NP (2005):

Mosaicplasty: Long-term follow-up.

Bull Hosp Jt Dis $\underline{63}, 54-62$

Trattning S, Plank C, Pinker K, Striessning G, Mlynarik V, Nöbauer I, Marlovits S (2004):

Bildgebende Diagnostik von Knorpelersatztherapien.

Radiologe 44, 748-755

Vallotton JA, Meuli RA, Leyvraz PF, Landry M (1995):

Comparison between magnetic resonance imaging and arthroscopy in the diagnosis of patellar cartilage lesions: a prospective study.

Knee Surg Sports Traumatol Arthrosc $\underline{3}, 157-162$

Weinstein SL (2000):

2000-2010: the bone and joint decade.

J Bone Joint Surg Am $\underline{\mathbf{8 2}}, 1-3$

Widuchowski W, Widuchowski J, Trzaska T (2007):

Articular cartilage defects: study of 25,124 knee arthroscopies.

Knee 14, 177-182

Yamashita F, Sakakida K, Suzu F, Takai S (1985):

The transplantation of an autogenic osteochondral fragment for osteochondritis dissecans of the knee.

Clin Orthop Relat Res 201, 43-50

Zacher J (2006):

Klinische Untersuchung des Kniegelenkes.

Orthop Unfallchir up2date ${ }^{1} \mathbf{1}, 33-56$ 


\section{Danksagung}

Ich möchte mich als erstes bei Herrn Prof. Dr. med. W. Schultz bedanken, der mir die Möglichkeit der Dissertation in seiner Abteilung eröffnet hat.

Weiterhin möchte ich mich bei Herrn Dr. med. M. Baums bedanken, der das Thema meiner Arbeit vorgeschlagen und mich bei der Anfertigung engagiert unterstützt hat.

Im Folgenden gilt mein besonderer Dank Hr. PD Dr. med. H.-M. Klinger für die strukturierenden, wissenschaftlichen Diskussionen und die Durchsicht der Arbeit, Hr. Dr. med. T. Nusselt für die Unterstützung hinsichtlich der Literaturrecherche, Frau Dipl.-Math. U. Schulz für die statistische Betreuung sowie Fr. G. Wolf-Bergk für die Illustrationen und Überlassung des Bildmaterials.

Besonders möchte ich mich bei Hr. Dr. med. F. Hoffmann bedanken, der bedeutend mein Interesse am Fach Orthopädie und Unfallchirurgie geweckt hat.

Außerdem bedanke ich mich bei Hr. Dr. med. J. Boehnke für die lehrreichen Gespräche und die Ermöglichung meiner zusätzlichen praktischen Einsätze auf dem operativen Gebiet der Orthopädie und Unfallchirurgie, welche bedeutend zum Verständnis der klinischen Problematik der Arbeit beitrugen. 


\section{Lebenslauf}

Nachdem ich am 26.08.1976 in Lauchhammer bei Senftenberg geboren wurde, besuchte ich zunächst von 1983 bis 1987 die Grundschule in Tettau, im Folgenden dann die Orientierungsstufe in Lindenau bis 1991. Die gymnasiale Ausbildung fand von 1991 bis 1996 am Gymnasium Lauchhammer statt und wurde mit dem Abitur abgeschlossen.

Im Sommer 1996 trat ich für 13 Monate meine Zivildienststelle an (Klinikum Niederlausitz $\mathrm{GmbH}$ ).

Von Januar bis August 1998 war ich danach als Praktikant im Kreiskrankenhaus Elsterwerda tätig.

Anschließend absolvierte ich bis Oktober 2001 eine dreijährige Berufsausbildung zum Krankenpfleger und schloss diese mit dem staatlichen Examen erfolgreich ab.

Nachfolgend arbeitete ich als Krankenpfleger zunächst in der ambulanten Krankenpflege. Anschließend nahm ich eine Tätigkeit als Krankenpfleger in der chirurgischen Klinik des Kreiskrankenhauses Finsterwalde auf.

Im April 2002 entschloss ich mich dazu, Humanmedizin zu studieren und trat das Studium der Humanmedizin an der Georg-August-Universität zu Göttingen an. Zusätzlich nahm ich eine Nebentätigkeit als Krankenpfleger im Niedersächsischen Landeskrankenhaus Göttingen (Fachkrankenhaus für Psychiatrie) auf, welche ich mit dem Beginn des Praktischen Jahres im Sommer 2005 beendete.

Das Praktische Jahr absolvierte ich in den Abteilungen Allgemein- und Transplantationschirugie, Rheumatologie und Nephrologie sowie Orthopädie der Universitätsmedizin Göttingen.

Die Approbation im Fach Humanmedizin an der Georg-August-Universität Göttingen erlangte ich nach dem 2. Teil der Ärztlichen Prüfung im Dezember 2008.

Seit Januar 2009 bin ich als Assistenzarzt im Fach Orthopädie und Unfallchirurgie in der Orthopädischen Klinik des Zentrums Chirurgie der Universitätsmedizin Göttingen tätig. 